\title{
An annotated checklist of Coccinellidae (Insecta, Coleoptera) with eight new records from the Kingdom of Saudi Arabia
}

\author{
Amin Al Ansi', Areej A. Alkhalaf², Hassan Fadl'³, Iftekhar Rasool', Hathal Al Dhafer' \\ I King Saud University Museum of Arthropods, Plant Protection Department, College of Food and Agriculture \\ Sciences, King Saud University, Riyadh, Saudi Arabia 2 Biology Department, College of Science, Princess Nou- \\ rah bint Abdulrahman University, Riyadh, Saudi Arabia 3 Entomology Departments, Faculty of Science, Ain \\ Shams University, Cairo, Egypt \\ Corresponding author: Amin Al Ansi (amohammed1@ksu.edu.sa)
}

Academic editor: J. Poorani | Received 29 September 2020 | Accepted 1 December 2020 | Published 21 December 2020

http://zoobank.org/4DD58069-8DFE-4444-8DBA-652DF0D671B8

Citation: Al Ansi A, Alkhalaf AA, Fadl H, Rasool I, Al Dhafer H (2020) An annotated checklist of Coccinellidae (Insecta, Coleoptera) with eight new records from the Kingdom of Saudi Arabia. ZooKeys 1006: 35-89. https://doi. org/10.3897/zookeys.1006.59123

\begin{abstract}
The members of Coccinellidae are among the most important biological control agents being used throughout the world. The objective of this study was to provide the current scenario of this family in Saudi Arabia. Collection surveys of ladybird individuals were conducted throughout Saudi Arabia. A total of 5400 specimens of Coccinellidae were collected belonging to 51 species in 19 genera and seven tribes: Sticholotidini (2 genera / 10 species), Coccidulini (1/3), Scymnini (4/24), Diomini (1/1), Hyperaspidini (1/3), Chilocorini (3/8), Noviini (1/3), Coccinellini (8/11), Epilachnini (2/2). One genus, Clitostethus, and eight species and subspecies, Cheilomenes lunata lunata (Fabricius, 1775), Clitostethus arcuatus (Rossi, 1794), Nephus ornatulus Korschefsky, 1931, N. nigricans (Weise, 1879), Pharoscymnus fleischeri (Weise, 1883), Novius yemenensis Raimundo \& Fürsch, 2006, Scymnus (Scymnus) scapuliferus Mulsant, 1850, and Stethorus endrodyi Fürsch, 1970, are reported as new records to Saudi Arabia. Two endemic species, Scymnus agrumi and S. arabicus, were recorded. This study also describes the geographical distribution for each species and the diagnostic characters for new records.
\end{abstract}

\section{Keywords}

Checklist, ladybird beetles, new records, Saudi Arabia, zoogeographical distribution

Copyright Amin Al Ansi et al. This is an open access article distributed under the terms of the Creative Commons Attribution License (CC BY 4.0), which permits unrestricted use, distribution, and reproduction in any medium, provided the original author and source are credited. 


\section{Introduction}

The members of Coccinellidae comprise one of the most important biological control agents being used throughout the world (Obrycki and Kring 1998). This family deserves its own superfamily Coccinelloidea in the suborder Polyphaga (Robertson et al. 2015) and represents approximately 6000 described species in 360 genera worldwide, of which approximately $90 \%$ are considered as predators (Vandenberg 2002; Hesler 2009; Magro et al. 2010). Monitoring the diversity of coccinellid species is crucial for integrated pest management programs and for incorporating the biological control of numerous arthropod pests. Coccinellids are relatively well known and commonly known as ladybird beetles in Australia, South Africa, and Great Britain and ladybugs in North America (Magro et al. 2010; Roy and Migeon 2010). These beetles can be found in various habitats, ranging from highly urbanized areas to the types of agro and natural ecosystems (Leather et al. 1999).

Before this study, a total of 56 species were reported from Saudi Arabia in 20 genera and 12 tribes (Kapur 1959; Shalaby 1962; Beccari 1971; Martin 1972; Fürsch 1979; Talhouk 1982; Walker and Pittaway 1987; Fürsch 1989; Raimundo and van Harten 2000; Kovár 2007). The first study on ladybird beetles of Saudi Arabia was conducted by Kapur (1959) with an initial record of 32 species from southwestern Arabia (southwestern KSA and Yemen). Subsequently, Hesler (1962) listed three species, Exochomus flavipes Thunberg, Epilachna hirta Thunberg, and Thea 16-notata (Mulsant 1850), from KSA. Beccari (1971) listed 10 species, Adonia variegata (Goeze, 1777), Chilocorus bipustulatus (L., 1858), Cheilomenes propinqua vicina Mulsant, 1850; Coccinella 7-punctata (L., 1758); C. 11-punctata (L., 1758); Epilachna chrysomelina (F., 1775); E. hirta (Thunberg, 1781); Exochomus flavipes (Thunberg, 1781); Scymnus punctillum (Weise, 1891); and Thea 16-notata (Mulsant, 1850), from KSA. Fürsch (1979) provided an identification key for 35 species, describing two new species, Nephus wittmeri Fürsch, 1979; and Pharoscymnus arabicus Fürsch, 1979, and one new subspecies, N. hiekei riyadhensis. Walker and Pittaway (1987) provided comprehensive comments, colour photographs, distributional information, and maps for four species of coccinellids occurring in Eastern Arabia. Fürsch (1989) also examined the groups of the speciesrich genus Scymnus of the Arabian Peninsula and recorded only six species from KSA, including two new species, S. arabicus Fürsch, 1989, and S. luxorensis Fürsch, 1989. Later, Raimundo and van Harten (2000) provided the description of the new species Serangium buettikeri Fürsch, 2000, from KSA and Yemen. The family Coccinellidae of the Palaearctic region was catalogued by Kovár (2007), who listed 41 species from KSA.

Several researchers have been exploring the diversity of Coccinellidae in the adjacent countries of Saudi Arabia. Yemen represents the highest diversity of coccinellids with 85 species (Raimundo and van Harten 2000; Raimundo et al. 2006). In the United Arab Emirates, 23 species have been reported to date (Raimundo et al. 2007; Gillett 2009), including a phytophagous species, Henosepilachna elaterii orientalis (Zimmermann, 1936). In Jordan, seven species of coccinellids, C. bipustulatus, Pharoscymnus ovoideus Sicard, 1929, Cheilomenes propinqua vicina, E. quadripustulatus, 
Scymnus subvillosus (Goeze, 1777), S. rubromaculatus (Goeze, 1777), and Oenopia conglobata (Linnaeus, 1758), have been recorded (Awameleh et al. 2009).

Syria has 20 recorded species of Coccinellidae (Khalil and Mourad 2005, 2006). In Egypt, 59 coccinellid species have been recorded (Alfieri 1976; Al Akkad 1979). In Palestine and adjacent areas, 71 species of coccinellids have been reported (Halperin et al. 1995). In the present study, the objective was to provide the current status of the family Coccinellidae for the KSA, the largest land mass of the Arabian Peninsula, by surveying the species of this family in selected regions of KSA, to identify the Coccinellidae specimens in the King Saud University Museum of Arthropods (KSMA) and Arabian National Museum of Arthropods, Ministry of Agriculture (ANMA), and the specimens that were collected during the present investigation.

\section{Materials and methods}

Collection surveys were conducted throughout the KSA (Figure 1) using beating sheets, malaise traps, sweep net, light traps, and sucking traps and by handpicking. Additional materials were examined from KSMA, ANMA, both in Riyadh, Saudi Arabia. Adult coccinellid beetles of large size were mounted immediately whenever possible using \#2 and \#3 pins (Monarch Brand from www.bioquip.com). Small-sized coccinellid beetles were mounted on small pieces of stiff paper, and some were preserved in $70 \%$ ethanol for dissection purpose and were deposited in KSMA.

For the dissection of genitalia, the method of Davis (2009) was followed with some modifications. Specimens were first relaxed by soaking in warm water for $10-15 \mathrm{~min}$. The abdomen was separated from the body under a stereomicroscope using fine needles

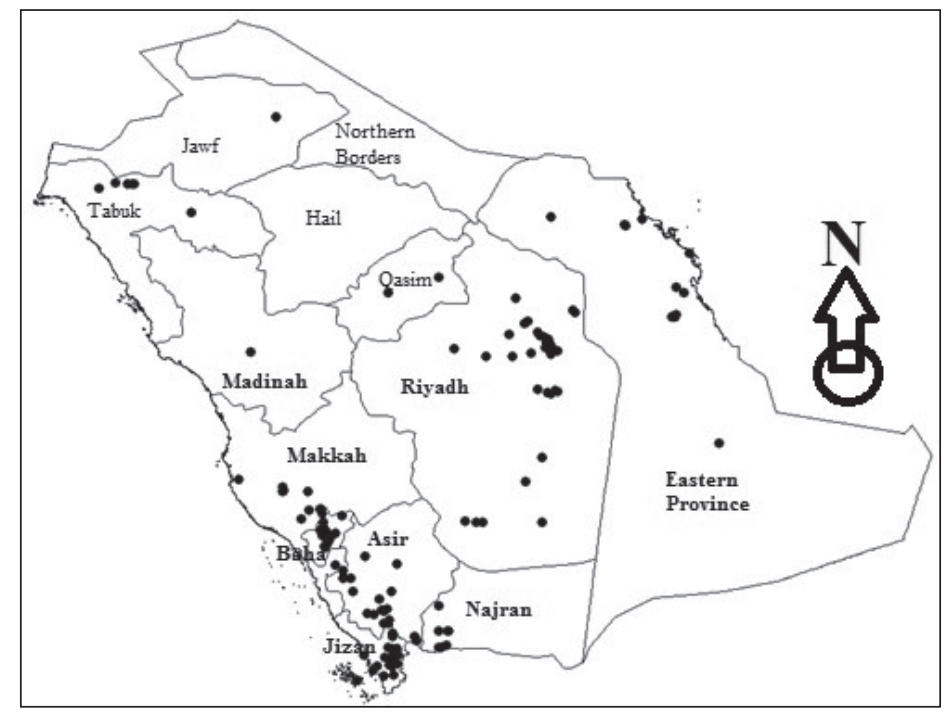

Figure I. Map of Saudi Arabia indicating collection localities. 
or forceps with small-sized tips. After extracting the genitalia (male genitalia or spermatheca) from the abdomen, they were placed in 10\% $\mathrm{KOH}$ solution for 5-10 min depending on the amount of tissues. After removing from the $\mathrm{KOH}$ solution, the genitalia were placed in water to neutralize any $\mathrm{KOH}$ for $2-5 \mathrm{~min}$, glued onto a small card using yellow glue (\# 1160 shellac gel), and placed underneath the specimen. Original illustrations were completed in addition to production of digital images using an AutoMontage version 1.2 with Micropublisher 5.0 RTV digital camera from the Q Imaging Company, Canada, installed on a Leica microscope type MZ 12.5 (Leica Company, Germany). All images were enhanced using Adobe Photoshop CS5.

The following information is provided for each species: current combinations of names and their synonymies, brief diagnosis (for new records only), remarks, material examined, country and world distribution (after Kovár 2007, for abbreviations see Table 1), and published records in KSA. The data for the material examined are arranged as follows: name of the province, followed by locality, the coordinates, elevation above sea level, the date of collection (months in Roman numerals), the collection methods (handpicking (HP), light trap (LT), malaise trap (MT), pitfall trap (PT), beating sheet (BS), sweeping net (SN), sucking (SU), and sticky traps (ST)), collector names, and the number of examined specimens, followed by sex $(\hat{\partial}, q$ for identified sex and ex $(s)$ for unidentified sex). A brief diagnosis of each new record species was added. New record species are marked with an asterisk. Geographical coordinates were obtained from GPS for specimens that were collected by the authors or obtained from the label data under the investigated individuals for specimens that were collected in KSMA or ANMA.

Species were identified using original description and all available literature reports such as Fürsch (1979; 1989), Raimundo and van Harten (2000), Raimundo et al. (2006), and Raimundo et al. (2007) and by examining the reference collection from ANMA and KSMA. Taxonomy on the tribe and genus levels follows Nedvěd (2020). Detailed accounts for each species and their ecological notes are also provided.

\section{Zoogeographical regions}

Acronyms for Zoogeographical regions used throughout text:

$\begin{array}{ll}\text { AFR } & \text { Afrotropical region } \\ \text { AUR } & \text { Australian region } \\ \text { ORR } & \text { Oriental region }\end{array}$

NAR Nearctic region

NTR Neotropical region

PAR Palaearctic region

\section{Results}

In total, 5400 specimens including both fresh collections from KSA and preserved materials in KSMA and ANMA were examined, of which 51 species were recognized in this investigation, including eight new records to the country (Table 2). Two endemic species Scymnus agrumi and S. arabicus were recorded. 
Table I. Acronyms for the countries are given following Kovár (2007).

\begin{tabular}{|c|c|c|c|}
\hline \multicolumn{4}{|c|}{ Acronyms of countries } \\
\hline Europe & & & \\
\hline AB: Azerbaijan & EN: Estonia & KZ: Kazakhstan & RO: Romania \\
\hline AL: Albania & FA: Faeroe Island & LS: Liechtenstein & RU: Russia \\
\hline AN: Andorra & FI: Finland & LT: Lithuania & SK: Slovakia \\
\hline AR: Armenia & FR: France & LU: Luxembourg & SL: Slovenia \\
\hline BE: Belgium & GB: Great Britain & MA: Malta & SP: Spain (Gibraltar) \\
\hline BH: Bosnia Herzegovina & GE: Germany & MC: Macedonia & SR: Svalbard (Spitzbergen) \\
\hline BU: Bulgaria & GG: Georgia & MD: Moldavia & $\begin{array}{c}\text { ST: Russia (South European } \\
\text { Territory) }\end{array}$ \\
\hline BY: Belarus & GR: Greece & NL: The Netherlands & SV: Sweden \\
\hline CR: Croatia & HU: Hungary & NR: Norway & SZ: Switzerland \\
\hline $\begin{array}{l}\text { CT: Russia (Central European } \\
\text { Territory) }\end{array}$ & IC: Iceland & $\begin{array}{c}\text { NT: Russia (North European } \\
\text { Territory) }\end{array}$ & TR: Turkey \\
\hline CZ: Czech Republic & IR: Ireland & PL: Poland & UK: Ukraine \\
\hline DE: Denmark & $\begin{array}{c}\text { IT: Italy (Sardinia, Sicily, San } \\
\text { Marino) }\end{array}$ & PT: Portugal & $\begin{array}{l}\text { YU: Yugoslavia (Serbia, } \\
\text { Montenegro) }\end{array}$ \\
\hline \multicolumn{4}{|l|}{ North Africa } \\
\hline AG: Algeria & EG: Egypt & MO: Morocco & TU: Tunisia \\
\hline CI: Canary Islands & LB: Libya & MR: Madeira Archipelago & \\
\hline \multicolumn{4}{|l|}{ Asia } \\
\hline AE: Arab Emirates & PAL: Palestine & NC: North Korea & SI: Egypt (Sinai) \\
\hline AF: Afghanistan & JA: Japan & OM: Oman & SY: Syria \\
\hline BA: Bahrain & JO: Jordan & PA: Pakistan & TD: Tajikistan \\
\hline $\mathrm{CH}$ : China & KI: Kyrgyzstan & QA: Qatar & TM: Turkmenistan \\
\hline CY: Cyprus & KU: Kuwait & RU: Russia & TR: Turkey \\
\hline FS: Russia (Far East) & KZ: Kazakhstan & SA: Saudi Arabia & UZ: Uzbekistan \\
\hline IN: Iran & MG: Mongolia & SC: South Korea & YE: Yemen including Socotra \\
\hline IQ: Iraq & NP: Nepal & $\begin{array}{c}\text { SD: India (Sikkim, Darjeeling } \\
\text { District) }\end{array}$ & \\
\hline
\end{tabular}

Table 2. Checklist of Coccinellidae species recorded from the Kingdom of Saudi Arabia.

\begin{tabular}{l} 
Species \\
\hline Brumoides adenensis Fürsch, 1987*** \\
Brumoides nigrosuturalis (Kapur, 1959) \\
Bulaea lividula bocandei Mulsant, 1850 \\
Cheilomenes lunata lunata (F., 1775) * \\
Cheilomenes lunata yemenensis Fürsch, 1989 \\
Cheilomenes propinqua vicina (Mulsant, 1850)
\end{tabular}

Chilocorus bipustulatus (L., 1758)

Chilocorus distigma Klug, 1835

Clitostethus arcuatus Rossi, 1794 **

Coccinella septempunctata (L., 1758)

Coccinella undecimpunctata menetriesi Mulsant, 1850

Diomus rubidus (Motschulsky, 1837)

Harmonia axyridis (Pallas, 1773)

Chnootriba elaterii orientalis (Zimmermann, 1936)

Henosepilachna hirta (Thunberg, 1781)

Hippodamia (Adonia) variegata (Goeze, 1777)

Hyperaspis polita Weise, 1855

Reference
Kováŕ 2007
Talhouk 1982

Kapur 1959; Fürsch 1979; Kovár 2007; Abdel-Dayem et al. 2017

Current study

Raimundo et al. 2006 reported from YE; Kovár 2007

Kapur 1959; Beccari 1971; Martin 1972; Fürsch 1979; Talhouk 1982; Kovář 2007

Beccari 1971; Martin 1972; Talhouk 1982; Kovár 2007 Kováŕ 2007

Current study

Beccari 1971; Martin 1972; Fürsch 1979; Talhouk 1982; Walker and

Pittaway 1987; Kovár 2007; Abdel-Dayem et al. 2017

Beccari 1971; Martin 1972; Talhouk 1982; Kovár 2007; Abdel-Dayem et al. 2017

Fürsch 1979; Kováŕ 2007; Abdel-Dayem et al. 2017 Biranvand et al. 2019

Kapur 1959; Beccari 1971; Martin 1972; Fürsch 1979; Walker and Pittaway 1987; Kovár 2007 Shalaby 1962; Beccari 1971

Beccari 1971; Talhouk 1982; Kovár 2007; El-Hawagry et al. 2013; Abdel-Dayem et al. 2017

Kováŕ 2007 


\begin{tabular}{l} 
Species \\
\hline Hyperaspis pumila pumila Mulsant, 1850 \\
Hyperaspis vinciguerrae Capra, 1929 \\
Nephus (Bipunctatus) conjunctus (Wollaston, 1870)*** \\
Nephus (Bipunctatus) wittmeri Fürsch, 1979*** \\
Nephus (Geminosipho) arcuatus Kapur, 1959 \\
Nephus (Geminosipho) fenestratus (Sahlberg, 1913)*** \\
Nephus (Nephus) crucifer Fleischer, 1900 \\
Nephus (sidis) hiekei (Fürsch 1965) \\
Nephus (sidis) levaillanti (Mulsant, 1850) \\
Nephus ornatulus Korscefsky, 1931* \\
Nephus nigricans (Weise, 1879)* \\
Oenopia oncina (Olivier, 1808)*** \\
Parexochomus (Exochomus) nigripennis Erichson, 1843
\end{tabular}

Parexochomus (Exochomus) nigromaculatus (Goeze, 1777)

Parexochomus pubescens (Kuster, 1848)

Parexochomus sjoestedti (Weise, 1910)***

Pharoscymnus arabicus Fürsch, 1979***

Pharoscymnus c-luteum (Sicard, 1907)

Pharoscymnus fleischeri (Weise, 1883)*

Pharoscymnus numidicus (Pic, 1900)

Pharoscymnus ovoideus Sicard, 1929

Pharoscymnus pharoides (Marsuel, 1868)***

Pharoscymnus setulosus (Chevrolat, 1861)

Pharoscymnus smirnovi Dobzhanskiy, 1927

Pharoscymnus tristiculus Sicard, 1907

Psyllobora bisoctonotata (Mulsant, 1850)

Novius argodi Sicard, 1909

Novius cardinalis (Mulsant, 1850)

Novius yemenensis Raimundo \& Fürsch, 2006*

Scymnus (Pullus) agrumi Fürsch, 1970

Scymnus (Pullus) arabicus Fürsch, 1989***

Scymnus (Pullus) auritus Thunberg, 1795***

Scymnus (Pullus) ebneri Weise, 1926

Scymnus (Pullus) latemaculatus Motschulsky, 1858

Scymnus (Pullus) luxorensis Fürsch, 1989

Scymnus (Pullus) subvillosus (Goeze, 1777)

Scymnus (Pullus) syriacus (Marsuel, 1868)

Scymnus (Pullus) yemenensis Kapur, 1959

Scymnus (Scymnus) interruptus (Goeze, 1777)

Scymnus (Scymnus) nubilus Mulsant, 1850

Scymnus(Scymnus) scapuliferus Mulsant, 1850*

Serangium buettikeri Fürsch, 2000

Stethorus gilvifrons (Mulsant, 1850)

Stethorus endrodyi Fürsch, 1970*

Tetrabrachys arabicus Kapur, 1948***

Tetrabrachys minutus (Pic, 1903)***

Tetrabrachys tenebrosus (Weise, 1910)***

Xanthadalia effusa rufescens (Mulsant, 1850)***

\section{Reference}

Kovár 2007

Martin 1972; Fürsch 1979; Abu-Thuraya 1982; Talhouk 1982; Kovár 2007; Abdel-Dayem et al. 2017

Fürsch 1979; Kovár 2007

Fürsch 1979; Kováŕ 2007; Abdel-Dayem et al. 2017

Fürsch 1979; Kováŕ 2007

Fürsch 1979; Kováŕ 2007

Fürsch 1979; Kováŕ 2007

Martin 1972; Fürsch 1979; Abu-Thuraya 1982; Talhouk 1982; Kovár 2007

Martin 1972; Abu-Thuraya 1982

Current study

Current study

Kovár 2007

Shalaby 1962; Beccari 1971; Martin 1972; Fürsch 1979; Talhouk 1982; Kovár 2007

Raimundo et al. 2006 reported from YE

Kapur 1959; Fürsch 1979; Kovár 2007; Abdel-Dayem et al. 2017 Kovár 2007

Fürsch 1979; Kováŕ 2007

Fürsch 1979; Kovár 2007

Current study

Fürsch 1979; Kovár 2007

Martin 1972; Abu-Thuraya 1982

Kovár 2007

Martin 1972; Fürsch 1979; Abu-Thuraya 1982; Kovár 2007

Kovár 2007

Abu-Thuraya 1982

Shalaby 1962; Beccari 1971; Fürsch 1979; Talhouk 1982; Kovár 2007

Martin 1972; Abu-Thuraya 1982

Abu-Thuraya 1982

Current study

Fürsch 1970; Martin 1972; Abu-Thuraya 1982; Talhouk 1982; Kovár 2007

Fürsch 1989; Kováŕ 2007 Kovár 2007

Martin 1972; Abu-Thuraya 1982; Talhouk 1982 Fürsch 1979

Fürsch 1989; Abdel-Dayem et al. 2017

Fürsch 1979; Kováŕ, 2007; Abdel-Dayem et al. 2017

Martin 1972; Talhouk 1982; Fürsch 1989; El-Hawagry et al. 2013; Abdel-Dayem et al. 2017

Fürsch 1979; Fürsch 1989; Kováŕ 2007; Abdel-Dayem et al. 2017

Fürsch 1979; Kovár 2007; Abdel-Dayem et al. 2017

Current study

Fürsch 2000; Kováŕ 2007

Martin 1972; Fürsch 1979; Abu-Thuraya 1982; Talhouk 1982; Kovár 2007

Current study

Kapur 1948; Fürsch 1979; Kovár 2007

Kapur 1948; Kovár 2007

Kapur 1948; Kovár 2007

Kovár 2007

Species with single marked asterisk ${ }^{*}$ are new species record; species marked with $\left({ }^{* *}\right)$ are new genera records; and species with $\left({ }^{* * *}\right)$ are found in old literature but not recorded in current work. 
Coccinellinae Latreille, 1807

Chilocorini Mulsant, 1846

Brumoides Chapin, 1965

\section{Brumoides adenensis Fürsch, 1987}

Brumoides adenensis Fürsch, 1987: 44.

Local distribution. This species was reported from KSA by Kovár (2007), but not collected during the present study.

World distribution. Asia: AE, IN, SA, and YE; AFR (Kovár 2007).

\section{Brumoides nigrosuturalis (Kapur, 1959)}

Brumus nigrosuturalis Kapur, 1959: 293.

Material examined. Asir: Al Majardah, Wadi Yabah, $19^{\circ} 16.27^{\prime} \mathrm{N}, 41^{\circ} 48.46^{\prime} \mathrm{E}, 411 \mathrm{~m}$, 2.VI.2012, BS, Al Ansi, A., $1 \overbrace{}^{\Uparrow} 1$; ; Al Majardah Thalooth Al Mandhar, Wadi Baqrah, $18^{\circ} 47.57^{\prime} \mathrm{N}, 42^{\circ} 01.12^{\prime} \mathrm{E}, 433$ m, 4.XI.2013, HP, Abdel-Dayem, M., 1ex; 12.X.2013, HP, El Torkey, A., 1ex; 12.X.2013, SN, Khan, S., 1ex; Maraba Wadi Ramlan, $17^{\circ} 47.18^{\prime} \mathrm{N}, 42^{\circ} 22.95^{\prime} \mathrm{E}, 180 \mathrm{~m}, 10.1 \mathrm{I} .2016$, SU, Al Ansi, A., 1ex; Maraba, Wadi Itwad, $17^{\circ} 48.25^{\prime} \mathrm{N}, 42^{\circ} 21.64^{\prime} \mathrm{E}, 149 \mathrm{~m}$, 8.II.2016, SU, Al Ansi, A., 4exs; 8.II.2016, SN, Fadl, H., 1ex; Maraba, Wadi Reem, $17^{\circ} 52.55^{\prime} \mathrm{N}, 42^{\circ} 16.66^{\prime} \mathrm{E}, 136 \mathrm{~m}$, 9.II.2016, SN, Fadl, H., 2exs; Jizan: Ahd Al Masareha Rd., $17^{\circ} 02.28^{\prime} \mathrm{N}, 42^{\circ} 52.38^{\prime} \mathrm{E}, 11 . \mathrm{III} .2010$, LT, Al Dhafer, H. and El Gharbawy, A., $10^{\top}$; Aiban, Wadi Dafa, $17^{\circ} 22.50^{\prime} \mathrm{N}, 43^{\circ} 04.53^{\prime} \mathrm{E}$, 870 m, 12.XI.2012, SN, Al Dhafer, H., 19 ; Sabya-Hurub Rd., $17^{\circ} 16.94^{\prime} \mathrm{N}, 42^{\circ} 17.54^{\prime} \mathrm{E}$, 97 m, 24.V.2012, SN, Al Ansi, A., 20; Aiban, Haqu Fifa, $17^{\circ} 07.95^{\prime} \mathrm{N}, 43^{\circ} 02.59^{\prime} \mathrm{E}$, 253 m, 11.XI.2012, BS, Fadl, H. and Abdel-Dayem, M., 1ð2ᄋ; Jizan-Abu Arish Rd, $17^{\circ} 04.25^{\prime} \mathrm{N}, 42^{\circ} 47.05^{\prime} \mathrm{E}, 24 . I I .2015$, SN, Al Harbi, M., 4exs; Jizan-Addarab Wadi Baiz, 17³7.56"N, 42²2.24"E, 249 m, 24.II.2015, HP, Al Harbi, M., 2ex.

Local distribution. This species was collected from southwest of KSA, Asir, and Jizan and was previously reported by Talhouk (1982) from Jizan.

World distribution. Asia: SA and YE; AFR (Kovár 2007).

\section{Chilocorus Leach, 1815}

Chilocorus bipustulatus (Linnaeus, 1758)

Coccinella bipustulata Linnaeus, 1758: 367. 
Remarks. This species is known as the biocontrol agent of the scale insect Parlatoria blanchardi (Targioni Tozzetti 1892), a date palm pest, and Aonidiella orientalis (Newstead 1894), a pest of Citrus spp. (Martin 1972), and is widely distributed throughout the world. In the present study, its adults were collected from date palm orchards on the grasses and shrubs under the palm tree and in the agroecosystem on the grasses.

Material examined. old collection from ANMA, Riyadh: Ad Diriyah, Education farm, $24^{\circ} 40^{\prime} \mathrm{N}, 46^{\circ} 35^{\prime} \mathrm{E}, 26 . I I I .1975,10^{\top}$, hand written label (date palm orchard); Wadi Hanifah, $24^{\circ} 45^{\prime} \mathrm{N}, 46^{\circ} 36^{\prime} \mathrm{E}$, 26.I.1977, Al Shaqaty, M., ANMA, 1ex; Riyadh, $24^{\circ} 43.01^{\prime} \mathrm{N}, 46^{\circ} 38.65^{\prime} \mathrm{E}$, 4.III.1979, Sarhan, K. and Shalaby, F., 29exs, hand written label [grasses]; V.1966, ANMA, 1ex.

Local distribution. This species was found in Riyadh region and listed by Martin (1972) and reported by Beccari (1971) from Riyadh and by Talhouk (1982) from Riyadh and Eastern region.

World distribution. Asia: AF, CY, FE, GAN, IN, IQ, PAL, JO, KI, KZ, LE, MG, SA, SI, SY, TR, UZ, and XIN; Europe: AB, AL, AR, AU, AZ, BE, BH, BY, CZ, CT, DE, EN, FI, FR, GB, GE, GG, GR, HU, IR, IT, LA, LT, LU, MA, MC, MD, NL, NR, NT, PL, PT, RO, SK, SL, SP, ST, SV, SZ, TR, UK, and YU; North Africa: AG, EG, LB, MO, MR, TU; AFR, and NAR (Kovár 2007).

\section{Chilocorus distigma Klug, 1835}

Chilocorus distigma Klug, 1835: 49.

Remarks. This is a rare species and found only in natural ecosystems. It was found only during February and March and was collected using a SN.

Material examined. Asir: Al Majardah, Wadi Qanunah, $1^{\circ} 24.67^{\prime} \mathrm{N}, 41^{\circ} 36.39^{\prime} \mathrm{E}$, 348 m, 11.III.2012, SN, Al Dhafer et al., 29407 71exs; 11.III.2012, LT, Al Dhafer et al., 1 + 7exs; Baisha, Wadi Targ, $19^{\circ} 37.37^{\prime} \mathrm{N}, 4^{\circ} 18.02^{\prime} \mathrm{E}, 1370 \mathrm{~m}, 14 . \mathrm{III} .2012$, SN, Al Dhafer et al., 1ex; Muhail, Wadi Hali, $18^{\circ} 30.12^{\prime} \mathrm{N}, 42^{\circ} 02.21^{\prime} \mathrm{E}, 440 \mathrm{~m}, 11 . I I .2016$, SN, Fadl, H., 1ex.

Local distribution. This species was collected from Asir and was previously listed by Kováŕ (2007).

World distribution. Asia: SA; AFR (Kovár 2007); YE (Raimundo and van Harten 2000).

\section{Parexochomus Barovskij, 1922}

\section{Parexochomus nigripennis (Erichson, 1843)}

Chilocorus nigripennis Erichson, 1843: 267.

Remarks. This species was reported as a predator of mealybugs (Talhouk 1982) and is known as the biocontrol agent of aphids such as Aphis nerii Boyer de Fonscolombe, 
1841, a pest of Nerium oleander L., and a predator on the soft scale insect Najacoccus serpentinus Green, 1919, a pest of Tamarix articulata Vahl (Martin 1972).

Material examined. Asir: Khamis Mushait, Wadi Bisha, $18^{\circ} 20.01^{\prime} \mathrm{N}, 42^{\circ} 42.13^{\prime} \mathrm{E}$, 1990 m 27.IV.2011, SN, Al Ansi, A., 1ex; Najran: Rijla, Wadi Najran, $17^{\circ} 31.56^{\prime} \mathrm{N}$, $44^{\circ} 13.65^{\prime} \mathrm{E}, 1257$ m, 15.I.2013, BS, Al Ansi et al., 4exs; Al Mofejah, $17^{\circ} 27.25^{\prime} \mathrm{N}$, $44^{\circ} 03.48^{\prime} \mathrm{E}, 1263 \mathrm{~m}$, 16.I.2013, BS, AlAnsi etal., $1{ }^{\Uparrow} 1$ 1 ; Baha: Wadi Bawah, $20^{\circ} 43.93^{\prime} \mathrm{N}$, $41^{\circ} 16.82^{\prime} \mathrm{E}, 1347$ m, 8.XI.2012, BS, Fadl, H., 1ex; Riyadh: Dirab, $23^{\circ} 30^{\prime} \mathrm{N}, 46^{\circ} 51^{\prime} \mathrm{E}$, 4.V.1987, SN, 1 ㅇ 1ex; 9.XII.1990, SN, 1ex; Al Kharj, 20²4'N, 4629'E, III.1980, SN, 10; Huraymila, $25^{\circ} 09^{\prime} \mathrm{N}, 46^{\circ} 08^{\prime} \mathrm{E}, 13 . \mathrm{II} .1988, \mathrm{SN}, \mathrm{Al}$ Dawood, A., 1 울exs; Al Waseel, $24^{\circ} 48.40^{\prime} \mathrm{N}, 46^{\circ} 30.42^{\prime} \mathrm{E}, 20 . X .2011$, SN, Al Rashedi, H., 1ex; Ad Diriyah, $24^{\circ} 40^{\prime} \mathrm{N}$, 463'ㄹ, 8.XII, SN, 1ex; 29.XII.2009, SN, Abdel-Gayyed, 1ex; Tabouk: Tabuk-Dhuba Rd., $28^{\circ} 18.39^{\prime} \mathrm{N}, 36^{\circ} 02.87^{\prime} \mathrm{E}, 824$ m, 15.IX.2011, SN, Al Ansi, A., 1ex; Qaseem: Buraydah, $26^{\circ} 12.954^{\prime} \mathrm{N}, 4^{\circ} 02.48^{\prime} \mathrm{E}, 633$ m, 17.IX.2011, SN, Al Ansi, A., 4exs; Riyadh, 24 43.01"N, 46 38.65"E, 1972, ANMA, 6exs; 18.IV.1978, SN, A.Talhouk et al., ANMA, 8exs, det. Fürsch, 1979; 2.II.1985, Abdulkader A., ANMA, 54 exs.

Local distribution. This species was found throughout the KSA and collected from Asir, Baha, Najran, Riyadh, Tabouk, and Qaseem during this study. It was also reported by Shalaby (1962) from Asir, listed by Martin (1972), and reported by Beccari (1971) from Asir, by Fürsch (1979) from Riyadh, and by Talhouk (1982) from Riyadh and Qaseem regions.

World distribution. Asia: AE, AF, IN, IQ, PAL, LE, PA, SA, SI, and SY; Europe: IT, PT, and SP; North Africa: AG, CI, EG, LB, MA, MO, and TU; AFR (Kovář 2007); YE (Raimundo and van Harten 2000).

\section{Parexochomus nigromaculatus (Goeze, 1777)}

Coccinella nigromaculata Goeze, 1777: 248.

Remarks. This species is known as a predator of mealybugs and aphids as mentioned by R. D. Pope (1969) on the label data and was collected from wild vegetation and natural habitats. It was found from February to May and in September.

Material examined. Asir: Abha, Sodah, $1^{\circ} 16.27^{\prime} \mathrm{N}, 42^{\circ} 21.52^{\prime} \mathrm{E}, 24 . I V .2011$, SN, Al Ansi, A., 1울 Khamis Mushait, Wadi Bisha, $18^{\circ} 20.01^{\prime} \mathrm{N}, 42^{\circ} 42.13^{\prime} \mathrm{E}$,

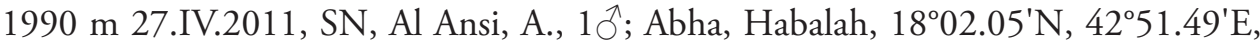
25.IV.2011, SN, Al Ansi, A., 1ex; Ahd Rifidh, $18^{\circ} 06.33^{\prime} \mathrm{N}, 42^{\circ} 53.82^{\prime} \mathrm{E}, 16.1 .2013$, SN, Al Ansi, A., 1우 Al Majardah, Wadi Khat, $1^{\circ} 05.37^{\prime} \mathrm{N}, 41^{\circ} 58.37^{\prime} \mathrm{E}, 13 . I I I .2012$, BS, Al Dhafer et al., 1q; 12.X.2013, SN, Sonbati, S., 1ex; Al Majardah, Wadi Qa-

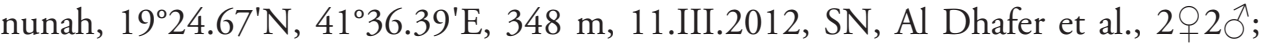
Muhail, Wadi Hali, $18^{\circ} 30.12^{\prime} \mathrm{N}, 42^{\circ} 02.21^{\prime} \mathrm{E}, 440 \mathrm{~m}, 11 . \mathrm{II} .2016$, SN, Fadl, H., 2exs; El Torkey, A., SN, 1ex; Baha: Wadi Galah, $20^{\circ} 08.08^{\prime} \mathrm{N}, 4^{\circ} 20.56^{\prime} \mathrm{E}, 16 . \mathrm{V} .2011$, SN, Fadl, H., 1 ; ; Al Mandaq, Wadi Turubah, $20^{\circ} 14.37^{\prime} \mathrm{N}, 41^{\circ} 15.23^{\prime} \mathrm{E}$, 3.VI.2012, BS, Al Ansi, A., 10; 27.IX.2013, Al Dhafer, H., 2exs; Biljuraishi, $19^{\circ} 55.76^{\prime} \mathrm{N}, 41^{\circ} 26.59^{\prime} \mathrm{E}$,

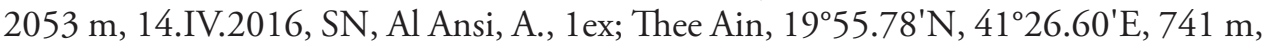


12.IV.2016, SU, Al Ansi, A., 2exs; 11.IV.2016, BS, Al Ansi et al., 2exs; Raghdan, $20^{\circ} 34.25^{\prime} \mathrm{N}, 41^{\circ} 45.11^{\prime} \mathrm{E}, 10 . I V .2016$, SN, Al Ansi, A., 1ex; Makkah: Taif, $21^{\circ} 08^{\prime} \mathrm{N}$, $40^{\circ} 58^{\prime}$ E, 10.IX.1969, R. D. Pope, ANMA, 3exs, on mealy bug.

Local distribution. Adults of this species were collected from different localities of Asir, Baha, and Makkah. This species was previously listed by Fürsch (1979).

World distribution. Asia: AF, CY, ES, FE, IN, IQ, PAL, KI, KZ, LE, PA, SY, TD, TR, UZ, WS, and XIN; Europe: AB, AL, AR, AU, BE, BH, BU, CR, CZ, DE, EN, FN, FI, FR, GB, GE, GG, GR, HU, IT, KI, KZ, MC, NL, NT, RO, SK, SL, SP, ST, SV, TR, UK, and YU (Kovár 2007); YE (Raimundo and van Harten 2000).

\section{Parexochomus pubescens (Kuster, 1848)}

Exochomus pubescens Kuster, 1848: 94.

Remarks. This species is widely distributed in the kingdom and found in both natural and agroecosystems throughout the year. It was collected from Acacia ehrenbergiana Hayne, A. gerrardii Benth., Calotropis procera (Aiton) W. T. Aiton, Lycium shawii Roem. and Schult., and Ziziphus nummularia (Burm.f.) Wight and Arn. (Abdel-Dayem et al. 2017). It is primarily known as a predator of aphids (Kapur 1959).

Material examined. Asir: Al Majardah, Wadi Al Talalie, $1^{\circ} 05.19^{\prime} \mathrm{N}, 41^{\circ} 47.78^{\prime} \mathrm{E}$,

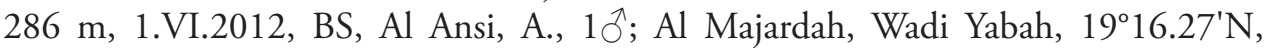
41\%48.46'E, 411, 2.VI.2012, BS, Al Ansi, A., 1우 2.VI.2012, LT, Abdel-Dayem, M., 1ex; 12.III.2012, LT, 1ex; 12.III.2012, SN, Al Dhafer et al., 1ex; Al Majardah, Wadi Khat, $19^{\circ} 05.37^{\prime} \mathrm{N}, 41^{\circ} 58.37^{\prime} \mathrm{E}, 31 . \mathrm{V} .2012$, BS, Al Ansi, A., 10`; 13.III.2012, BS, Al

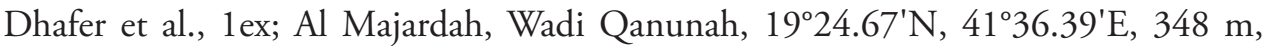
11.III.2012, BS, Al Dhafer et al., 10’; 11.III.2012, SN, Al Dhafer et al., 1ex; Abha, Sodah, $18^{\circ} 16.27^{\prime} \mathrm{N}, 42^{\circ} 21.52^{\prime} \mathrm{E}, 24 . I V .2011, \mathrm{SN}$, Al Ansi et al., 1ex; Al Majardah, Thalooth Al Mandhar, Wadi Baqrah, $18^{\circ} 47.57^{\prime} \mathrm{N}, 42^{\circ} 01.12^{\prime} \mathrm{E}, 433 \mathrm{~m}, 31 . \mathrm{V} .2012$, BS, Al Ansi, A., 1ex; 10.XI.2012, BS, Fadl, H., 11exs; 13.III.2012, BS, Abdel-Dayem et al., 1ex; Baha: Al Mandaq, Wadi Turubah, $20^{\circ} 14.37^{\prime} \mathrm{N}, 41^{\circ} 15.23^{\prime} \mathrm{E}$, 3.VI.2012, LT, Al Ansi, A., $10^{\top} 2$; ; 27.IX.2013, Al Dhafer, H., 2exs; Thee Ain, $19^{\circ} 55.78^{\prime} \mathrm{N}, 41^{\circ} 26.60^{\prime} \mathrm{E}$,

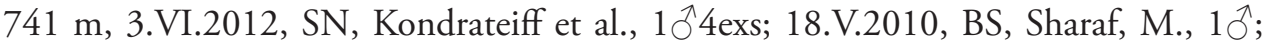
10.III.2012, BS, Al Dhafer et al., 1ex; 11.IV.2016, BS, Al Ansi et al., 1§ో; Al Mikhwah, $19^{\circ} 49.44^{\prime} \mathrm{N}, 41^{\circ} 22.85^{\prime} \mathrm{E}, 430 \mathrm{~m}$, 7.III.2013, BS, Al Harbi et al., 2exs; Wadi Al Zaraeib, 15.V.2010, BS, Sharaf, M., 1ex; Jizan: Al Aydabi, Wadi Qasi, $17^{\circ} 26.52^{\prime} \mathrm{N}, 42^{\circ} 57.32^{\prime} \mathrm{E}$,

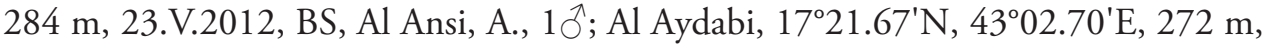

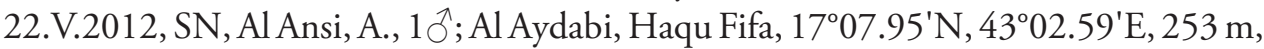
21.V.2012, BS, Al Ansi, A., 1 ${ }^{\top} 1$ ex; Aiban-Sabya Rd., Wadi Shahdan, $17^{\circ} 28.27^{\prime} \mathrm{N}$, 4251.19'E, 433 m, 13.XI.2012, BS, Fadl, H., 10¹1exs; Abu Arish-Al Aridhah Rd., Wadi Jizan, $17^{\circ} 01.34^{\prime} \mathrm{N}, 42^{\circ} 56.73^{\prime} \mathrm{E}, 127$ m, 11.XI.2012, BS, Fadl, H., 7exs; JizanAbuArish Rd, $17^{\circ} 04.25^{\prime} \mathrm{N}, 42^{\circ} 47.05^{\prime}$ E, 24.II.2015, SN, Al Harbi, M., 1ex; Sabya, City Center, $17^{\circ} 14.27^{\prime} \mathrm{N}, 42^{\circ} 46.42^{\prime} \mathrm{E}, 43$ m, 23.V.2012, BS, Al Ansi, A., 4exs; Ayban-Sab- 
ya Rd., Wadi Qasi, $17^{\circ} 26.52^{\prime} \mathrm{N}, 42^{\circ} 57.32$ 'E, 284 m, 12.XI.2012, BS, Fadl, H., 2exs; Aiban, Haqu Fifa, $17^{\circ} 07.95^{\prime} \mathrm{N}, 43^{\circ} 02.59^{\prime} \mathrm{E}, 253$ m, 11.XI.2012, BS, Fadl, H., 1ex; Al Aridhah, Wadi Al Rad, $17^{\circ} 04.10^{\prime} \mathrm{N}, 43^{\circ} 04.33^{\prime} \mathrm{E}, 192 \mathrm{~m}, 21 . \mathrm{V} .2012$, BS, Al Ansi, A., 1ex; Hurub, Wadi Qasi, $17^{\circ} 26.52^{\prime} \mathrm{N}, 42^{\circ} 57.32^{\prime} \mathrm{E}, 284$ m, 24.V.2012, BS, Al Ansi, A., 1ex; Najran: Wadi Shuaib Barran, $17^{\circ} 28.94^{\prime} \mathrm{N}, 4^{\circ} 05.52^{\prime} \mathrm{E}, 1325 \mathrm{~m}, 16 . \mathrm{I} .2013$, BS, $\mathrm{Al}$ Ansi et al., 1ex; Hubuna, Lahumah, $17^{\circ} 50.47^{\prime} \mathrm{N}, 4^{\circ} 16.82^{\prime} \mathrm{E}, 1212 \mathrm{~m}$, 14.I.2013, BS, Al Ansi et al., 1ex; Riyadh: Huraymila, $25^{\circ} 09^{\prime} \mathrm{N}, 46^{\circ} 08^{\prime} \mathrm{E}, 1 . \mathrm{XII} .1988, \mathrm{SN}$, 1ex; Shuaib Huraymila, $25^{\circ} 06.10^{\prime} \mathrm{N}, 46^{\circ} 04.22^{\prime} \mathrm{E}, 804 \mathrm{~m}$, 22.IV.2012, BS, Al Ansi, A., 5exs; Rawdhet Khoraim, $25^{\circ} 22.98^{\prime} \mathrm{N}, 47^{\circ} 16.71^{\prime} \mathrm{E}, 559 \mathrm{~m}$, 89exs were collected by BS and SU on branches of A. ehrenbergiana, A. gerrardii, C. procera, L. shawii and $Z$. nummularia; through IX-XII 2011; I-V, XI.2012; I, III.2013. Qaseem: Buraydah, $26^{\circ} 12.954^{\prime} \mathrm{N}, 44^{\circ} 02.48^{\prime} \mathrm{E}, 633 \mathrm{~m}$, 17.IX.2011, SN, Al Ansi et al., $1{ }^{\Uparrow} 1$ ㅇ․

Local distribution. This species was collected from a variety of habitats in Asir, Baha, Jizan, Najran, Riyadh, and Qaseem provinces and previously reported by Kapur (1959) from Hejaz; Fürsch (1979) from Riyadh, Asir, Jizan, and Makkah; and AbdelDayem et al. (2017) from Riyadh.

World distribution. Asia: AF, IN, IQ, PAL, and SA; Europe: FR, GR, IT, and SP; North Africa: AG, EG, LB, MO, and TU; AFR (Kovár 2007); YE (Raimundo and van Harten 2000) and AE (Raimundo et al. 2007).

\section{Parexochomus sjoestedti Weise, 1910}

Parexochomus sjoestedti Weise, 1910: 260.

Remarks. This is a rare species and Afrotropical in distribution. There is no literature to confirm its presence in Saudi Arabia, except the Catalogue of Kovár (2007), and it was also not collected during this study.

World distribution. A: SA; AFR (Kovár 2007).

\section{Tetrabrachini Kapur, 1948}

\section{Tetrabrachys Kapur, 1948}

All the three species are restricted to the Arabian Peninsula and some Afrotropical countries. Although several researchers (Kapur 1948; Fürsch 1979; Kovár 2007) have reported these three species from the KSA, not a single species was recorded during the present study.

\section{Tetrabrachys arabicus Kapur, 1948}

Tetrabrachys arabicus Kapur, 1948: 329. 
Remarks. This species is restricted to the Arabian Peninsula. It was recorded from the KSA by Kapur (1948), Fürsch (1979), and Kovár (2007) but not found during the present study.

Local distribution. Al Hejaz (Kapur 1948); Asir (Fürsch 1979).

World distribution. Asia: SA and YE (Kovár 2007).

\section{Tetrabrachys minutus (Pic, 1903)}

Lithophilus minutus Pic, 1903: 170.

Remarks. This is also a rare species that was recorded from the KSA by Kapur (1948) and Kovár (2007) without definite localities. It was not found in the present study.

World distribution. Asia: SA; North Africa: TU (Kovár 2007).

\section{Tetrabrachys tenebrosus (Weise, 1910)}

Lithophilus tenebrosus Weise, 1910: 53.

Remarks. This is a rare species found in the Arabian Peninsula and Africa; it was recorded from the KSA by Kapur (1948) and from the KSA and Yemen by Kovár (2007) without definite localities. It was not found in the present study.

World distribution. Asia: SA and YE; AFR (Kovár 2007).

\section{Coccinellini Latreille, 1807}

Bulaea Mulsant, 1850

\section{Bulaea lividula bocandei Mulsant, 1850}

Bulaea bocandei Mulsant, 1850: 71, 1016.

Remarks. Members of Bulaea are phytophagous and feed on the pollen of plants. This species was collected on Aerva javanica (Burm. f.) Juss. ex Schult (Amaranthaceae), and it was also known to feed on leaves and pollen (Raimundo et al. 2007).

Material examined. Asir: Al Majardah, Wadi Khat, $19^{\circ} 05.37^{\prime} \mathrm{N}, 41^{\circ} 58.37^{\prime} \mathrm{E}$, 31.V.2012, BS, Al Ansi, A., 1 $912 \mathrm{exs}$; Wadi Qanunah, $19^{\circ} 24.67^{\prime} \mathrm{N}, 41^{\circ} 36.39^{\prime} \mathrm{E}$, 348 m, 11.V.2011, SN, Fadl, H.; Setyaningrum et al., 1ex; Wadi Yabah, $19^{\circ} 16.27^{\prime} \mathrm{N}$, 414․46'E, 411, 15.IV.2016, SN, Soliman, A., 2exs; Maraha, Wadi Reem, $17^{\circ} 52.55^{\prime} \mathrm{N}, 42^{\circ} 16.66$ 'E, 136 m, 9.II.2016, SN, Fadl, H., 2exs; Maraha, Wadi Ramlan, $17^{\circ} 47.18^{\prime} \mathrm{N}, 42^{\circ} 22.95^{\prime} \mathrm{E}, 180$ m, 9.II.2016, SN, Al Ansi, A., 3exs; SU, Al Ansi, A.,

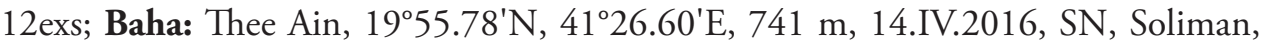


A., 1ex; Jizan: Abu Arish Wadi Jizan, $17^{\circ} 01.34^{\prime} \mathrm{N}, 4^{\circ} 56.73^{\prime} \mathrm{E}, 127 \mathrm{~m}, 21 . \mathrm{V} .2012$,

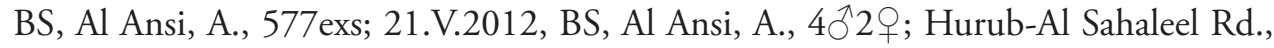
$17^{\circ} 47.34^{\prime} \mathrm{N}, 42^{\circ} 85.65^{\prime} \mathrm{E}, 456$ m, 24.V.2012, BS, Al Ansi, A., 7exs; Al Aydabi Jaorat Aiban, $17^{\circ} 25.53^{\prime} \mathrm{N}, 43^{\circ} 03.50^{\prime} \mathrm{E}, 343 \mathrm{~m}, 23 . \mathrm{V} .2012$, SN, Al Ansi, A., 2exs; 11.XI.2012, BS, Fadl, H., 21 exs; Al Aydabi Haqu Fifa, 17²1.54'N, 4302.62'E, 237 m, 21.V.2012, SN, Al Ansi, A., 1ex; Aiban Sabya Rd. Wadi Shahadan, 13.XI.2012, BS, Fadl, H., Gexs; Jizan-Addarab Wadi Baiz, $17^{\circ} 37.56^{\prime} \mathrm{N}, 4^{\circ} 22.24^{\prime} \mathrm{E}, 249$ m, 24.II.2015, HP, Al Harbi, M., Gexs; Jizan, 10.II.1982, SN, Talhouk et al., ANMA, 22exs; 25.XII.1980, SN, Talhouk et al., ANMA, 3exs; 15.VIII.1982, SN, Talhouk et al., ANMA, 1ex. Riyadh: Al Uyaynah, $24^{\circ} 53^{\prime} \mathrm{N}, 46^{\circ} 22^{\prime} \mathrm{E}, 24 . \mathrm{V} .2007$, SN, 1ex; Rawdhet Khoraim, $25^{\circ} 22.98^{\prime} \mathrm{N}$, $47^{\circ} 16.71$ 'E, 559 m, 29.VIII.2013, BS, 1ex, on Ziziphus nummularia; Wadi Ad Dawasir, $20^{\circ} 25.83^{\prime} \mathrm{N}$, $44^{\circ} 56.53^{\prime} \mathrm{E}, 641 \mathrm{~m}$, 17.I.2013, BS, Al Ansi et al., 10exs.

Local distribution. This species was found in Asir, Baha, Jizan, and Riyadh, but the majority of specimens of B. lividula bocandei were collected from several localities of Jizan province. This species was previously reported by Kapur (1959) from Asir, by Fürsch (1979) from the Eastern region and by Abdel-Dayem et al. (2017) from Riyadh.

World distribution. Asia: AE, IN, IQ, PAL, JO, PA, SA, SY, and YE; North Africa: $A G, E G$, and MO; AFR (Kovár 2007).

\section{Cheilomenes Chevrolat, 1836}

\section{Cheilomenes lunata lunata (Fabricius, 1775) *}

Figure 2

Coccinella lunata lunata Fabricius, 1775: 86.

Diagnosis. Pronotum is black with white anterolateral angles and anterior margin; elytra are black with large and rounded yellow-reddish spots. Pattern of spots on each elytron: separated spots on the shoulder, but mostly shoulder spot united with scutellar spot to a semi-circular spot outside the humeral callus; two rounded spots in the elytral centre: one near the suture and one in a more marginal position; two spots on the posterior half of elytron: one forming an inverted horizontal $\mathrm{C}$ and the other one stretching along the posterolateral elytral margin.

Material examined. Asir: Abha, Raydah, $18^{\circ} 12.10^{\prime} \mathrm{N}, 42^{\circ} 24.54^{\prime} \mathrm{E}, 2578 \mathrm{~m}$, 16.IV.2016, SN, Soliman, A., 1 9 ; Rijal Alma, Wadi Sabin, $17^{\circ} 48.25^{\prime} \mathrm{N}, 42^{\circ} 21.64^{\prime} \mathrm{E}$,

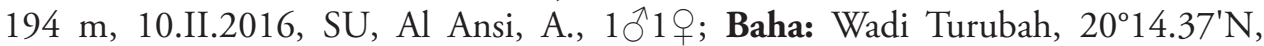
41 15.23'E, 10.V.2011, SN, Fadl et al., 10ิ.

Local distribution. This species was previously recorded from Yemen by Raimundo et al. (2006) and collected from Asir and Baha provinces during the present study.

World distribution. North Africa: EG; AFR (Kovár 2007); Asia: YE (Raimundo et al. 2006) and a new country record for the KSA. 


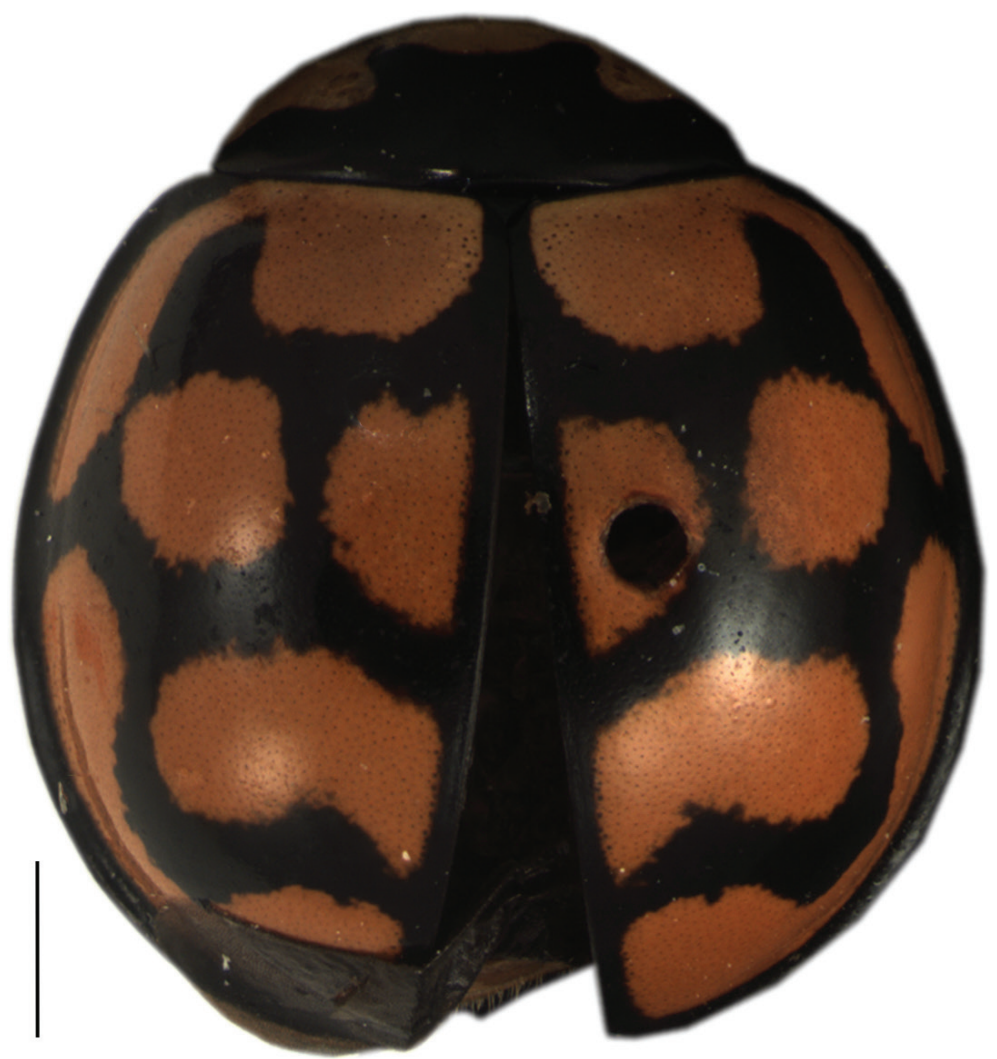

Figure 2. Dorsal view of Cheilomenes lunata lunata. Scale bar: $1 \mathrm{~mm}$.

\section{Cheilomenes lunata yemenensis Fürsch, 1989}

Cheilomenes lunata yemenensis Fürsch, 1989 (2): 37-38.

Remark. A rare subspecies that is confined only to the Arabian Peninsula and was described from Yemen. In Saudi Arabia, it is widely distributed in both natural habitats and agroecosystems.

Materials examined. Asir: Abha, Sodah, $18^{\circ} 16.27^{\prime} \mathrm{N}, 42^{\circ} 21.52^{\prime} \mathrm{E}, 24 . \mathrm{IV} .2011$,

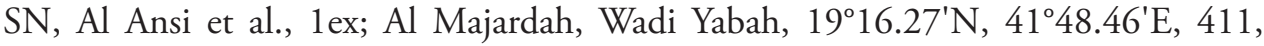
2.VI.2012, BS, Al Ansi, A., 10̂; 12.III.2012, SN, Al Dhafer et al., 1ex; Wadi Turubah, $20^{\circ} 14.37^{\prime} \mathrm{N}, 41^{\circ} 15.23^{\prime} \mathrm{E}, 14 . \mathrm{V} .2011$, SN, Fadl et al., 10'; 14.X.2010, SN, Al Dhafer et al., 1\%; Abha, Raydah, $18^{\circ} 12.10^{\prime} \mathrm{N}, 4^{\circ} 24.54^{\prime} \mathrm{E}, 2578 \mathrm{~m}$, 8.II.2016, SU, Al Ansi, A., 1ex; 16.IV.2016, SN, Soliman, A., 2exs; Al Ansi et al., SU, 1ex; Rijal Alma, Wadi Sabin, $17^{\circ} 48.25^{\prime} \mathrm{N}, 42^{\circ} 21.64^{\prime} \mathrm{E}, 194$ m, 10.II.2016, SN, Fadl, H., 1ex; Baha: Wadi Dhahyan Dam, 200 $5.51^{\prime} \mathrm{N}, 41^{\circ} 24.11^{\prime} \mathrm{E}, 883 \mathrm{~m}$, 9.V.2011, SN, Fadl et al., 1ex; Ghabat Amdhan, $20^{\circ} 06.32^{\prime} \mathrm{N}, 41^{\circ} 17.15^{\prime} \mathrm{E}, 19 . \mathrm{V} .2010$, SN, El Torkey, A., 1ex; Shada Al Aala, $19^{\circ} 50.58^{\prime} \mathrm{N}, 41^{\circ} 18.69^{\prime} \mathrm{E}, 1666$ m,14.IV.2016, HP, Abdel-Dayem, M., 1ex; Thee Ain, $19^{\circ} 55.78^{\prime} \mathrm{N}, 41^{\circ} 26.60^{\prime} \mathrm{E}, 741 \mathrm{~m}$, 12.IV.2016, SN, Al Ansi, A., 1ex; Ragh- 
dan, $20^{\circ} 34.25^{\prime} \mathrm{N}, 41^{\circ} 45.11^{\prime} \mathrm{E}, 10 . I V .2016$, SU, Al Ansi, A., 1ex; Jizan: Fifa, $17^{\circ} 16^{\prime} \mathrm{N}$, $43^{\circ} 05^{\prime} \mathrm{E}$, 1.II.1983, SN, Talhouk et al., ANMA, 15exs; 25.I.1983, SN, ANMA, 38exs; Makkah: Taif Rd., 12.X.2010, SN, Al Dhafer et al., 2exs.

Local distribution. Specimens of this species were collected from Asir, Baha, Jizan, and Makkah provinces. It was previously reported from the KSA by Kovár (2007) but with no distributional range.

World distribution. Asia: This species is confined to the Arabian Peninsula and was recorded only from SA and YE (Kovář 2007).

\section{Cheilomenes propinqua vicina (Mulsant, 1850)}

Cydonia propinqua vicina Mulsant, 1850: 411.

Remark. This species is associated with a wide range of both natural habitats and agroecosystems, predating on other arthropods. It was known to predate particularly on aphids such as Rhopalosiphum maidis (Fitch, 1856), a pest of Setaria italica (L.) (Martin 1972; Raimundo and van Harten 2000).

Materials examined. Asir: Khamis Mushait, Wadi Bisha, $18^{\circ} 20.01^{\prime} \mathrm{N}, 42^{\circ} 42.13^{\prime} \mathrm{E}$, 1990 m 27.IV.2011, SN, Al Ansi, A., 1ex; Al Majardah Wadi Yabah, 19¹6.27'N, 41ํํ․ 46'E, 411, 12.V.2012, Al Ansi, A., LT 2exs, SN 5exs; Al Majardah, Wadi Al Talalie, $19^{\circ} 05.19^{\prime} \mathrm{N}, 41^{\circ} 47.78^{\prime} \mathrm{E}, 286$ m, 12.III.2012, SN, Al Ansi, A., 2exs; Al Majardah, Wadi Khat, $1^{\circ} 05.37^{\prime} \mathrm{N}, 41^{\circ} 58.37^{\prime} \mathrm{E}$, 13.III.2012, BS, Al Dhafer et al., 2exs; Khamis Mushait, $18^{\circ} 18^{\prime} \mathrm{N}, 42^{\circ} 45^{\prime} \mathrm{E}$, 4.X.2005, SN, 1ex; Wadi Abha, $18^{\circ} 22.03^{\prime} \mathrm{N}, 42^{\circ} 50.82^{\prime} \mathrm{E}$,

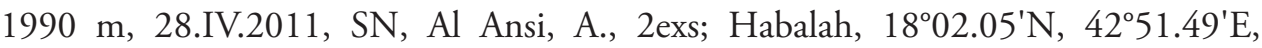
25.IV.2011, SN, Al Ansi, A., 1ex; Al Majardah Wadi Qanunah, 19²4.67'N, 4136.39'E, 348 m, 11.III.2012, SN, Al Dhafer et al., 4exs; 11.V.2011, SN, Fadl, H.,

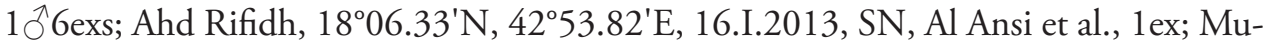
hail, Wadi Hali, $18^{\circ} 30.12^{\prime} \mathrm{N}, 42^{\circ} 02.21^{\prime} \mathrm{E}, 440$ m, 11.II.2016, SU, Al Ansi, A., 4exs; Rijal Alma, Wadi Sabin, $17^{\circ} 48.25^{\prime} \mathrm{N}, 42^{\circ} 21.64^{\prime} \mathrm{E}, 194 \mathrm{~m}$, 10.II.2016, SN, Fadl, H., 1ex; Maraha, Wadi Reem, $17^{\circ} 52.55^{\prime} \mathrm{N}, 42^{\circ} 16.66^{\prime} \mathrm{E}, 136$ m, 9.II.2016, SU, Al Ansi, A., 5exs; Maraha, Wadi Ramlan, $17^{\circ} 47.18^{\prime} \mathrm{N}, 42^{\circ} 22.95^{\prime} \mathrm{E}, 180 \mathrm{~m}, 10 . \mathrm{II} .2016$, SU, Al Ansi et al., 2exs; Maraha, Wadi Itwad, $17^{\circ} 48.25^{\prime} \mathrm{N}, 42^{\circ} 21.64^{\prime} \mathrm{E}, 149 \mathrm{~m}$, 8.II.2016, SU, Al Ansi, A., 2exs; Baha: Wadi Turubah, $20^{\circ} 14.37^{\prime} \mathrm{N}, 41^{\circ} 15.23^{\prime} \mathrm{E}, 14 . \mathrm{V} .2011, \mathrm{SN}$, Fadl, H., 2exs; 14.III.2012, SN, Fadl, H., 1ex; Thee Ain, 1955.78'N, 41 $26.60^{\prime} \mathrm{E}, 741 \mathrm{~m}$, 11.V.2011, SN, Fadl, H., 8exs; 14.IV.2016, SN, Soliman, A., 1ex; 12.IV.2016, SU, Al Ansi, A., 3exs; SN, 1ex; 15.V.2011, SN, Fadl, H., $2 \precsim 8$ exs; 3.VI.2012, HP, Abdel-Day-

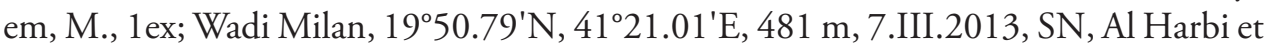
al., 2exs; Al Mikhwah-Muhail Rd. Hadba Hamra, 19²13.19'N, 41 ${ }^{\circ} 46.67^{\prime} \mathrm{E}, 374$ m, 15.

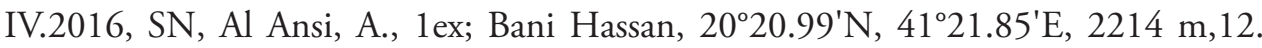
IV.2016, SN, Soliman, A., 1ex; Jizan: Jizan-Ahd Al Masareha Rd., $17^{\circ} 02.28^{\prime} \mathrm{N}$, 42 $52.38^{\prime} \mathrm{E}$, 12.III.2010, SN, Al Dhafer et al., 1ex; 11.III.2010, LT, Al Dhafer et

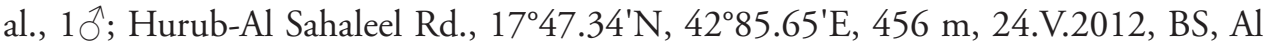
Ansi, A., 2exs; Jizan city, $16^{\circ} 54^{\prime} \mathrm{N}, 42^{\circ} 29^{\prime} \mathrm{E}, 10 . I I .1982$, SN, Talhouk et al., ANMA, 
34exs; same data except 10.II.1981, SN, ANMA, 11exs; same data except 1.II.1983, SN, ANMA, 1ex; same data except 20.I.1979, SN, ANMA, 3exs; same data except 16.VIII.1977, SN, Fathy S., ANMA, 1ex.; Makkah: Jedah-Makkah Rd., 2125.14'N, 39¹9.49'E, 15.XII.2006, SN, 2exs; Baha-Taif Rd. Bani Malik, Wadi Rakhmat, $20^{\circ} 42.15^{\prime} \mathrm{N}, 40^{\circ} 59.46^{\prime} \mathrm{E}, 1616 \mathrm{~m}, 20^{\circ} 42.15^{\prime} \mathrm{N}, 40^{\circ} 59.46 ' \mathrm{E}, 1616 \mathrm{~m}, 4 . \mathrm{VI} .2012, \mathrm{SN}$, Al Ansi, A., $1 \overbrace{}^{\Uparrow} 2$ exs; Najran: Rijla Wadi Najran, $17^{\circ} 31.56^{\prime} \mathrm{N}, 44^{\circ} 13.65^{\prime} \mathrm{E}, 1257 \mathrm{~m}$, 15.I.2013, BS, Al Ansi et al., 3exs; Wadi Shuaib Barran, $17^{\circ} 28.94^{\prime} \mathrm{N}, 44^{\circ} 05.52^{\prime} \mathrm{E}$, 1325 m, 16.I.2013, BS, Al Ansi et al., 3ð2exs; Riyadh: Riyadh, SN, Abu Yaman, 1ex; Education Farm, $24^{\circ} 40^{\prime} \mathrm{N}, 46^{\circ} 35^{\prime} \mathrm{E}$, 26.III.1975, SN, 1ex; Ad Diriyah, $24^{\circ} 40^{\prime} \mathrm{N}$, $46^{\circ} 35^{\prime} \mathrm{E}, 15 . X I I .2005$, SN, 1ex; Dirab 2330'N, 46ํำ'E, 13.III.2003, SN, 1ex.

Local distribution. Specimens of this species were collected from Asir, Baha, Jizan, Makkah, Najran, and Riyadh and previously reported by Kapur (1959) from Asir and Al Hajaz, by Beccari (1971) from Jizan and Madinah, by Fürsch (1979) from Asir, and by Talhouk (1982) from Jizan, and listed by Martin (1972).

World distribution. Asia: IQ, PAL, LE, SA, and YE; North Africa: AG, EG, and LB; AFR (Kovár 2007).

\section{Coccinella L., 1758}

\section{Coccinella septempunctata L., 1758}

\section{Coccinella septempunctata L., 1758: 365.}

Remark. This species is widely distributed around the world and found in a wide range of habitats. Both adults and larvae are voracious predators of aphids (Sattar et al. 2008). It is a biocontrol agent of Aphis craccivora C.L. Koch, 1854, and Therioaphis trifolii (Monell, 1882), pests of alfalfa, Medicago sativa L. (Martin 1972).

Material examined. Eastern Province: Al Ahsa, $22^{\circ} 17.53^{\prime} \mathrm{N}, 50^{\circ} 40.46^{\prime} \mathrm{E}$, 4.III.2011, SN, Al Ansi, A. 1ex, on alfalfa; 4.III.2011, SN, Al Ansi, A., 1ex; Riyadh: Al Ammariah, $24^{\circ} 49^{\prime} \mathrm{N}, 46^{\circ} 26^{\prime} \mathrm{E}$, 28.I.2009, SN, Widman, A., 1ex, on alfalfa; 28.I.2008, SN, Valenza, B., 2exs, on alfalfa; 28.I.2009, SN, Setyaningrum, H., 1 우 ex; Ad Diriyah, $24^{\circ} 40^{\prime} \mathrm{N}, 46^{\circ} 35^{\prime} \mathrm{E}, 29 . X .2008$, SN, Al Ahmari, A., 19, on alfalfa; 29.X.2008, SN, Bel Hareth, H., 2exs, on alfalfa; 16.I.2009, SN, Setyaningrum, H., 1ex; 29.X.2008, SN, Al Sebaiei, M., 4exs, on alfalfa; 29.X.2008, SN, Al Qahtani, M., 1ex, on alfalfa; 17.IV.2003, SN, Al Habeeb, A., 1ex, on alfalfa; 17.X.2003, SN, Al Saiari, M., 1ex; 30.XII.1989, SN, Amro, 1ex, on pepper; 21.II, SN, Al Essa, M., 1ex; Al Kharj, $20^{\circ} 24^{\prime} \mathrm{N}, 4^{\circ} 29^{\prime} \mathrm{E}, 24 . I I I .2010$, SN, Al Othman, A., 1ex; 26.XI.2008, SN, Sofan, A., 3exs, on water melon; 26.XI.2008, SN, Widman, A., 10ึ, on alfalfa; 14.XI.2007, SN, 1ex; 26.X.1991, SN, Al Ahmadi, F., 1ex, on alfalfa; 9.III.2001, SN, Al Baqami, A., 1ex; Al 16.X, SN, Al Sharaf, M., 1ex; Al Uyaynah, $24^{\circ} 53^{\prime} \mathrm{N}, 46^{\circ} 22^{\prime} \mathrm{E}, 10 . I I I .2010$, SN, Al Hashel, A., 1ex; 30.XI.2010, SN, Al Ansi, A., 1ex, on corn; 11.XI.2008, SN, Setyaningrum, H., 3exs; 11.XI.2008, SN, Setyaningrum, H., 1ex; 26.II.2004, SN, Droshy, R., 1ex, on alfalfa; 7.III.2004, SN, Jasim, D., 10; 4.V.2005, SN, Turkestani, S., 1ex; 2.X.2003, SN, Al Haj, M., 1ex; 9.IV.2008, SN, Al Tork, 1ex, on alfalfa; Al 
Waseel, $24^{\circ} 48.40^{\prime} \mathrm{N}, 46^{\circ} 30.42^{\prime} \mathrm{E}, 12 . \mathrm{XI} .2008$, SN, Al Qahtani, M., 1ex, on alfalfa; 1.XII.2010, SN, Al Abdul Muhsen, M., 1ex; 12.XI.2008, SN, Namoosy, S., 2exs, on alfalfa; 20.XI.2011, SN, Al Mater, A., 1ex; 11.X, SN, Al Farhan, 1ex, on alfalfa; 20.XI.2011, SN, Al Sahwan, K., 1ex; 12.XI.2008, SN, Baziyad, A., 3exs, on alfalfa; Riyadh, SN, Abu Yaman, 1ex; Dirab, $23^{\circ} 30^{\prime}$ N, 46 $51^{\prime}$ E, 30.XII.2009, SN, Setyaningrum, H., 1ex, on grass; 7.III.2011, SN, Salah, A., 1ex, on fig plant; 25.I.1990, SN, 1ex, on alfalfa; 10.X.2011, SN, Al Amro, S., 1ex; Hautet Bani Tamim, $23^{\circ} 27.26^{\prime} \mathrm{N}$, 46눈.13'E, 4.IV.2008, SN, Al Dhafer et al., 2exs; Otaiqah, $24^{\circ} 35.46^{\prime} \mathrm{N}, 46^{\circ} 42.24^{\prime} \mathrm{E}$, 16.XI.1989, SN, 1ex, on Squash; Rawdhet Khoraim, $25^{\circ} 22.98^{\prime} \mathrm{N}, 47^{\circ} 16.71^{\prime} \mathrm{E}$, 559 m, 11.IV.2011, SN, 1ex; Al Uayainnah, 25.II.2015, SN, Al Sofayan, M., 1ex; Riyadh, 15.III.1978, SN, Talhouk et al., ANMA, 5exs;Tabuk: Tabuk-Dhuba Rd., $28^{\circ} 18.39^{\prime} \mathrm{N}, 36^{\circ} 02.87^{\prime} \mathrm{E}, 824 \mathrm{~m}$, 15.IX.2011, SN, Al Ansi, A., 1ex; Tabuk-Madinah Rd., 28²3.47'N, 3651.96'E, 808 m, 14.IX.2011, SN, Al Ansi, A., 1ex.

Local distribution. Its specimens were collected from Eastern Province, Riyadh and Tabouk; this species was previously listed by Martin (1972), Talhouk (1982), and Walker and Pittaway (1987) and reported by Beccari (1971) from Riyadh, by Fürsch (1979) from Riyadh, and by Abdel-Dayem et al. (2017) from Riyadh.

World distribution. Asia: AF, ANH, BEI, BT, CY, FE, FUG, GAN, GUA, GUI, GUX, HEB, HEI, HEN, HUB, HUN, IN, IQ, PAL, JA, JIA, JIL, JIX, JO, KA, KI, KU, KZ, LE, LIA, NIN, NMO, MG, NC, NP, PA, QIN, SA, SCH, SC, SD, SHA, SHG, SHN, SHX, SI, SY, TAI, TD, TIA, TM, TR, UP, UZ, XIN, XIZ, YUN, and ZHE; Europe: $A B, A L, A N, A R, A U, B E, B H, B U, B Y, C R, C T, C Z, D E, E N, F I$, FR, GB, GE, GG, GR, HU, IR, IT, LA, LS, LT, LU, MC, MD, NL, NR, NT, PL, RO, SK, SL, SP, ST, SV, SZ, TR, UK, and YU; North Africa: MR; AFR, NAR, and ORR (Kovář 2007).

\section{Coccinella undecimpunctata menetriesi Mulsant, 1850}

Coccinella undecimpunctata menetriesi Mulsant, 1850: 104.

Remark. This species with several subspecies is widely distributed throughout the world. Both adults and larvae are known as predators of aphids. It is commonly found in the KSA throughout the year. It is known as a good biocontrol agent of Aphis craccivora C.L. Koch, 1854, and Therioaphis trifolii (Monell, 1882), pests of alfalfa, Medicago sativa L. (Martin 1972).

Material examined. Asir: Wadi Abha, $18^{\circ} 22.03^{\prime} \mathrm{N}, 42^{\circ} 50.82^{\prime} \mathrm{E}, 1990 \mathrm{~m}, 28$. IV.2011, SN, Al Ansi et al., 1ex; Al Majardah, Wadi Yabah, $19^{\circ} 16.27^{\prime} \mathrm{N}, 41^{\circ} 48.46^{\prime} \mathrm{E}$, 411, 2.VI.2012, SN, Al Ansi, A.M 1ex; 12.III.2012, SN, Al Dhafer et al., 1ex; Wadi Targ, $1^{\circ} 37.37^{\prime} \mathrm{N}, 42^{\circ} 18.02^{\prime} \mathrm{E}, 1370 \mathrm{~m}, 14 . I I I .2012$, HP, Abdel-Dayem, M., 1ex; Baha: Wadi Bawah, $20^{\circ} 43.93^{\prime} \mathrm{N}, 41^{\circ} 16.82^{\prime} \mathrm{E}, 1347 \mathrm{~m}, 20^{\circ} 44.98^{\prime} \mathrm{N}, 41^{\circ} 14.85^{\prime} \mathrm{E}$, 1310 m, 8.XI.2012, SN, Fadl, H., 1ex; 8.XI.2012, SN, Abdel-Dayem, M., 1ex; Wadi Turubah, $20^{\circ} 14.37^{\prime} \mathrm{N}, 41^{\circ} 15.23^{\prime} \mathrm{E}, 14 \mathrm{X} .2010$, SN, Al Dhafer et al., 1ex; Taif, $21^{\circ} 08^{\prime} \mathrm{N}$, 405', 20.I.1993, SN, Al Saad, B., 1ex; Baha-Taif Rd. Bani Malik Wadi Rakhmat, 
$20^{\circ} 42.15^{\prime} \mathrm{N}, 40^{\circ} 59.46^{\prime} \mathrm{E}, 1616 \mathrm{~m}, 20^{\circ} 42.15^{\prime} \mathrm{N}, 40^{\circ} 59.46^{\prime} \mathrm{E}, 1616 \mathrm{~m}, 4 . \mathrm{VI} .2012, \mathrm{SN}$, Al Ansi, A., 1ex; Eastern Province: Abu Hadriyah-Dammam Rd. North Rawabi Farm, $27^{\circ} 34.21^{\prime} \mathrm{N}, 48^{\circ} 50.53^{\prime} \mathrm{E}, 15 \mathrm{~m}, 3 . \mathrm{III} .2011, \mathrm{SN}, 1 \mathrm{ex}$; Hafuf, $25^{\circ} 15^{\prime} \mathrm{N}, 49^{\circ} 32^{\prime} \mathrm{E}, 19$. II.1980, SN, 2exs; Dammam-Riyadh Rd., $250 \mathrm{~km}$ before Riyadh, $25^{\circ} 50.00^{\prime} \mathrm{N}$, $49^{\circ} 50.23^{\prime} \mathrm{E}, 174 \mathrm{~m}, 25^{\circ} 50.00^{\prime} \mathrm{N}, 49^{\circ} 50.23^{\prime} \mathrm{E}, 174 \mathrm{~m}, 5 . I I I .2011$, SN, 21 exs; An Nuayriyah, $27^{\circ} 25.26^{\prime} \mathrm{N}, 48^{\circ} 27.20^{\prime} \mathrm{E}, 67 \mathrm{~m}$, 2.III.2011, SN, 3exs; 2.III.2011, SN, 20exs; Abu Hadriyah-Dammam Rd., $27^{\circ} 34.21^{\prime} \mathrm{N}$, 48 $8^{\circ} 50.53^{\prime} \mathrm{E}, 15 \mathrm{~m}, 2 . \mathrm{III} .2011$, SN, 3exs; Al Ahsa, $22^{\circ} 17.53^{\prime} \mathrm{N}, 50^{\circ} 40.46$ 'E, 4.III.2011, SN, 5exs, on alfalfa; 4.III.2011, SN, 6exs, on weeds; 4.III.2011, SN, 14exs, on weeds; 4.III.2011, SN, 3exs, on weeds; Qatif, $26^{\circ} 45^{\prime} \mathrm{N}, 49^{\circ} 58^{\prime} \mathrm{E}$, 20.VIII, SN, Al Salem, S., 1ex; same V.1966, ANMA, 1ex, predator on Parlatoria blanchardii; 4.III.1979, SN, Sarhan et al., ANMA, 29exs; 25.IX.1978, SN, Talhouk et al., ANMA, 13exs; 15.IX.1978, SN, Shalbay, F., ANMA, 1ex; SN, Buttikeri, W., ANMA, 1ex; Najran: As Sullayil-Najran Rd., $340 \mathrm{~km}$ before Najran, $18^{\circ} 01.35^{\prime} \mathrm{N}, 42^{\circ} 45.81^{\prime} \mathrm{E}, 619$ m, 13.I.2013, SN, Al Ansi et al., 1ex; Riyadh: Rawdat Khoraim, $25^{\circ} 25.94^{\prime} \mathrm{N}, 47^{\circ} 13.86^{\prime} \mathrm{E}, 520 \mathrm{~m}$, collected through the year 2011-2013 using SN, BS, PT, SU and LT on Rhazya stricta, L. shawii, Ziziphus nummularia, and A. gerrardii, Al Dhafer et al., 66exs; Wadi Namar, $24^{\circ} 32.18^{\prime} \mathrm{N}, 46^{\circ} 34.60^{\prime} \mathrm{E}$, 6.IV.2012, SN, Al Othman, A., 1ex; 29.II.2012, SN, Al Ansi, A., 5exs; Otaiygah, 16.XI.1989, SN, 6exs, on alfalfa; Al-Quway'iyah, $24^{\circ} 20^{\prime} \mathrm{N}, 45^{\circ} 09^{\prime} \mathrm{E}$, 18.V.2012, SN, Al Ghamdi, R., 1ex; Al Muzahimiyah, Al Khararah, $2^{\circ} 24.21^{\prime} \mathrm{N}, 46^{\circ} 14.40^{\prime} \mathrm{E}, 17 . \mathrm{IV} .2012$, SN, Al Dhafer et al., 4exs; 3.IV.2012, SN, Al Dhafer et al., 8exs; 21.II.2012, SN, Al Dhafer et al., 2exs; 17.IV.2012, LT, Al Dhafer et al., $10^{\Uparrow} 1$ q 2 exs; 17.IV.2012, SN, Abdel-Dayem, M., 1ex; 3.X.2011, SN, Al Dhafer et al., 1ex; 25.VI, Jubari, 2exs; 26.IV.2011, SN, Al Deryhim et al., 1ex; Shuaib Huraymila, $25^{\circ} 06.10^{\prime} \mathrm{N}, 46^{\circ} 04.22^{\prime} \mathrm{E}, 804 \mathrm{~m}, 22 . I V .2012$,

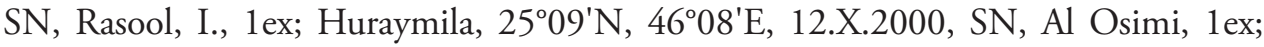
13.II.1988, SN, Dawood, 1ex, on weeds; Ilaisha, $24^{\circ} 37.58^{\prime} \mathrm{N}, 46^{\circ} 41.14^{\prime} \mathrm{E}, 18 . X I I .1989$, SN, 1ex, on cowpea; Sajir, $24^{\circ} 51.23^{\prime} \mathrm{N}, 4^{\circ} 42.39^{\prime} \mathrm{E}, 17 . X I .2011$, SN, Setyaningrum,

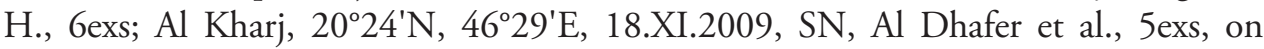
Calotropis; 24.III.2010, SN, Al Mutairi, T., 3exs; 24.III.2010, SN, Al Hori, M., 1ex; 20.XI.2009, SN, Sofan, A., 1ex; 26.XI.2008, SN, Namosi, S., 1ex; 2.V.2007, SN, 2exs;14.XI.2007, SN, Ahmed, 2exs; 8.XI.1986, SN, 1ex, on alfalfa; 26.XI.2008, SN, Valenza, B., 1ex; 26.X.1996, SN, Al Ahmed, F., 3exs on alfalfa; 17.IV.2003, SN, Al Habeeb, A., 2exs, on alfalfa; 9.III.2011, SN, Al Hazmi, H., 1ex, on weeds; 26.X.2008, SN, Setyaningrum, H., 3exs, on vegetables; 1.IV.2009, SN, Al Zahrani, M., 2exs; 1.IV.2009, SN, Al Amri, A., 2exs; 2.I.2008, SN, Al Bohmi, 1ex; 24.III.2010, SN, Al Dawsari, M., 1ex; 16.X, SN, Mostafa, 1ex; 26.X.1991, SN, Al Ahmed, F., 2exs, on Alfalfa; III.1980, SN, 1ex, on wheat; Al Kharj Al Rafei, 20²4'N, 46 29'E, 16.X.2003, SN, Al Hamad, M., 1ex; 29.XII.2002, SN, Saif, A., 1ex, on sorghum; 4.IV.2007, SN, Al Shehri, 1ex; 4.IV.2007, SN, Al Hamzi, A., 1ex; 17.V.2007, SN, Al Greed, S., 1ex; Al Haair, $24^{\circ} 27^{\prime} \mathrm{N}, 46^{\circ} 51^{\prime} \mathrm{E}, 31 . I .1989$, SN, Salem, 14exs, on barley; 25.IX.1990, SN, Salem, 1ex, on weeds; 14.V.1989, SN, Salem, 1ex on weeds; 13.XI, SN, Al Mutlaq, 1ex, on eggplant; 13.XI.1990, SN, Al Qarni, 1ex; 13.XI, SN, Al Jaberi, 1ex, on vegetables; 1.II.2009, SN, Setyaningrum, H., 1ex, on alfalfa; 2.XII.1988, SN, Al Fuhaid, M., 1ex; Wadi Hanifah, $24^{\circ} 45^{\prime}$ N, 463'ㄹ, 26.I.1977, SN, Al Shaqaty, M., 1ex; Al Ammariah, 
$24^{\circ} 49^{\prime} \mathrm{N}, 4^{\circ} 26^{\prime} \mathrm{E}$, 13.III.2013, SN, Al Qahtani, F., 1ex; 4.X.2012, SN, Hussain, S., 3exs; 4.X.2012, SN, Rasool, I., 2exs; 22.II.2012, SN, Al Atwi, M., 1ex; 23.I, SN, Al Awadhi, A., 4exs; 8.IV.2007, SN, Bin Jaber, A., 1ex; 11.X, SN, 1ex, on Weeds; 15. XII.2011, SN, Al Omar, A., 1ex; 18.XI.2009, SN, Sofan, A., 1ex; 18.XI.2009, SN, Setyaningrum, H., 2exs; 28.I.2009, SN, Arya, 2exs; 28.I.2009, SN, Setyaningrum, H., 1ex; 21.X.2010, SN, Koko, 4exs; 30.XII.2008, SN, Abdel-Gayed, 2exs; 11.XI.2009, SN, Al Ansi, A., 2exs; 1.I.2010, SN, Koko, 1ex; 1.I.2010, SN, Single, A., 1ex; 22.V.2011, SN, Al Ahmari, S., 2exs; Hawtet Bani Tammem, 2328.10'N, 46³6.35'E, 7.V.2012, SN, Al Ansi, A., 15exs; 7.V.2012, SN, Al Dhafer et al., 2exs; 10.XI.2011, SN, Al Abdullah, I., 1ex; 21.III.2008, SN, Al Dhafer et al., M., 2exs; 4.IV.2008, SN, Al Dhafer et al.,

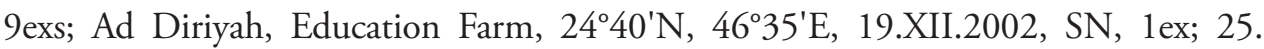
XII.2010, SN, Al Otaibi, F., 1ex; Ad Diriyah, $24^{\circ} 40^{\prime} \mathrm{N}, 46^{\circ} 35^{\prime} \mathrm{E}$, 20.III.2009, Husain, M., 1ex; 20.IV.1992, SN, Al Asmari, D., 1ex; 11.X.2010, SN, Abdel-Gayed, 1ex; 30. XII.1989, SN, Amro, 4exs, on vegetables; 21.X.1993, SN, Hatem, 1ex; 5.V, SN, Aseri, M., 1ex; 19.III.1989, SN, 1ex, on alfalfa; 2.III.1989, SN, 1ex, on grasses; 29.III.1989, SN, 2exs, on grasses; 24.III, SN, 5exs, on vegetables; 8.X.2009, SN, Single, A., 1ex; 22.XII.2002, SN, 1ex; 9.XII, SN, 1ex, on alfalfa; 7.XI.1990, SN, Al Saleh, 1ex, on vegetables; 16.X.1989, SN, 5exs, on weeds; 23.X.1989, SN, 1, on alfalfa; 7.XI, SN, Al Qarni, 1ex, on vegetables; 5.XII.2010, SN, Al Turkey, I., 1ex; 2.VII.2009, SN, Al Zahrani, M., 1ex; 24.XII.1991, SN, Ghazi, 2exs; 7.III.2012, SN, Basahih, J., 1ex; 4.VII, SN, Al Qathban, M., 1ex, on alfalfa; 5.X.2004, SN, Al Otaibi, B., 1ex, on alfalfa; 8.XII, SN, Al Saab, 1ex, on alfalfa; 2.XII.1988, SN, Al Fuhaid, M., 1ex, feed on aphid; 16.X.2003, SN, Al Dhubaib, N., 2exs; 4.V.2005, SN, Turkestani, S., 1ex; III.1980, 1ex, on date palm, det. T. G. Vazirani, 1983; 15.XII.1973, SN, Atiyat Allah, A., 1ex; 29.X.2008, SN, Al Ahmari, A., 1ex; 30.XI.2011, SN, Al Dehaaesh, N., 1ex; XI.1989, SN, 2exs, on alfalfa; 16.III.1989, SN, Salem, 1ex, on alfalfa; 7.VII.1991, SN, Al Fudhail, M. 1ex, on alfalfa; 6.X.2003, SN, Al Hammad, M., 1ex; 12.IV.1987, SN, 1ex, on alfalfa; 5.VI.1993, SN, Aziz, 1ex; 21.I, SN, Al Harbi, S., 2exs, on alfalfa; 30.X.1989, SN, 2exs, on alfalfa; 29.X.2008, SN, Al Qahtani, M., 3exs, on alfalfa; 16.X.2000, SN, Al Khurigi, 3exs; 7.XI.1990, SN, Al Mutlaq, 1ex, on vegetables; 20.IV.1992, SN, Saif, A., 1ex, on alfalfa; 15.IV.2000, SN, Hatan, 1ex; 28.IX.2003, SN, Al Otaibi, A., 2ex; 16.I, SN, Moshal, 1ex, on alfalfa; 14.IV.1993, SN, Aziz, 1ex; 12.IV.1993, SN, Aziz, 1ex; 15.IV.1993, SN, 1ex, on crops; 19.VIII, SN, Aiman, 2ex; 8.II.2001, SN, Al Qarni, M., 1ex; 23.XI.2011, SN, Al Kassem, A., 1ex; 24.III, 1ex, on vegetables; 16.X.1989, SN, 1ex, on grasses; 13.XI.1989, SN, 1ex, on wheat; 29.III.1989, 1ex, on weeds; XII.1980, SN, 1ex, on alfalfa; Irqah, 8.XII.2010, SN, Al Dhahbani, I., 1ex; Dirab, $23^{\circ} 30^{\prime} \mathrm{N}$, 46 $51^{\circ} \mathrm{E}, 5 . V .1987$, SN, 1ex, on alfalfa; 24.II.1986, LT, 1ex; 1.V.1997, 1ex; 25.XI.1990, Amr, 1ex, on alfalfa,; 17.XI., Al Bathi, F., 1ex, on alfalfa; 9.I, 2exs, on alfalfa; 16.V.2007, SN, Sharaf Addeen, A., 2exs; 16.III.2005, SN, 1ex;18.III.2004, SN, Yaser, 1ex; 18.IV.1982, 1ex, on sorghum; 13.III.2003, Al Habeeb, A., 1ex, on alfalfa; 2.I.1987, SN, 1ex; 4.VIII, SN, Al Osimi, 1ex; 24.III.1987, 1ex, on alfalfa; 5.I.1987, 1ex, on alfalfa; 19.V.2009, SN, Al Moteb, M., 1ex; IX.1989, 2exs, on alfalfa; 7.IV, 5exs, on alfalfa; 14.IV.1987, 1ex, on alfalfa; 9.V.1991, SN, Al Fudhail, M., 1ex; 3.III.1987, Amro, 3exs, on alfalfa; 31.III.1987, 3exs, on alfalfa; 17.X.2011, SN, Al Khudhairi, H., 
3exs; 10.X.2011, SN, Al Amro, S., 2exs; 30.XII.2009, Setyaningrum, H., 3exs, on grasses; 7.IV.1987, 4exs, on alfalfa; 17.X.2011, SN, Jubran, R., 1ex; 21.IV, 1ex, on alfalfa; 12.IV.1993, SN, Al Saleh, 1ex; 25.XII.2003, SN, Al Yaaqub, A., 1ex; 13.III.2003, SN, Al Shathri, B., 2exs; 9.V, SN, Moshal, 2exs, on alfalfa; 10.III.1986, SN, 1ex;13.X.1986, 2exs, on alfalfa; 10.XI.1986, 1ex, on weeds; 25.XI.1990, Amro, 1ex, on alfalfa; 2.III.1989, Amro, 1ex, on grasses; 7.IV.1987, Amro, 2exs, alfalfa; 24.X.1992, 1ex, on alfalfa; 15.VIII.1993, Aseri, M., 1ex, on alfalfa; 19.XII.2010, SN, Setyaningrum, H. and Al Dhafer, H., 1ex; 30.XII.2009, SN, Sofan, A., 6exs; 31.X.2010, SN, Al Ansi, A., 9exs; 17.X.2010, SN, Setyaningrum, H., 1ex; 14.III.2010, SN, Al Dhafer, H.; El Gharabawy, A. and El Torkey, A., 2exs; Al Waseel, 24⒋40'N, 46 30.42'E, 1. XII.2010, SN, Al Abdul Muhsen, N., 1ex; 11.X.2012, SN, Hussain, S., 1ex; 1.X.2012, SN, Sonbati, S., 1ex; 14.III.2012, SN, Al Atwi, Z., 1ex; 14.III.2012, SN, Al Edwani, M., 1ex; 11.X, 1ex, on weeds; 11.X, Aseri, H., 1ex, on alfalfa; 12.XI.2008, Al Qahtani, M. 2exs, on alfalfa; 25.III.2004, Al Aati, 1ex, on alfalfa; 7.XI.2007, SN, Al Salman, 1ex; 20.XI.2011, SN, Al Sahwan, K., 1ex; 12.XI.2008, SN, Namosi, S., 2exs; 1.XII.2010, S.N.,Al Harbi, S., 1ex; 27.V.2009, SN, Adyatma, 1ex; 21.XI.2009, SN, Al Ansi, A.,

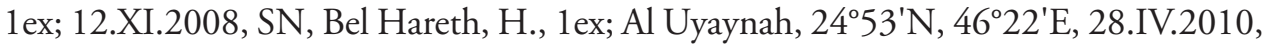
SN, Al Yusif, A., 1ex; 10.III.2010, SN, Al Salem, B., 1ex; 28.IV.2010, SN, Al Hashel, A., 1ex; 10.III.2010, SN, Al Hashel, A., 1ex; 1.XII.2010, SN, Al Ghunaim, A., 1ex; 3.XI.2010, SN, Al Ghunaim, A., 1ex; 27.IX.2003, SN, Mostafa, 2exs; 3.XI.2010, SN, Abdul Mohsen, 1ex; 30.XI.2010, SN, Al Dhafer, H. and Al Ansi, A., 1ex; 9.IV.2008, SN, Al Turk, 3exs; 26.II.2004, SN, Derwish, K., 1ex; 9.IV.2008, SN, Al Shahrani, 1ex; 27.II.2008, SN, Al Shamrani, 1ex; 12.X.2011, Al Husani, 2exs, on alfalfa; 24.X.2007, SN, Al Salman, 1ex; 18.IX.2011, SN, Al Amro, S., 2exs; 18.IX.2011, SN, Al Turki, I., 1ex; 12.X.2011, SN, Al Omar, A., 3exs; 19.X.2011, SN, Al Omar, A., 1ex; 8.VI.1993, 1ex, on alfalfa; 25.IX.1993, 1ex, on alfalfa; 6.IV.1993, 1ex, on weeds; 12.X.2011, SN, Al Hamad, Y., 1ex; 15.XII.2010, SN, Al Dhahbani, I., 2exs; 5.VIII, SN, Aiman, 1ex; 7.II.2005, SN, Al Oqail, 1ex; 8.II.2012, SN, Al Ghamdi, M., 1ex; 8.II.2012, SN, Al Wajaan, A., 1ex; 18.IX.2011, SN, Al Amro, S., 1ex; Wadi Ad Dawasir-As Sullayil Rd., $20^{\circ} 25.30^{\prime} \mathrm{N}, 45^{\circ} 05.65^{\prime} \mathrm{E}, 632 \mathrm{~m}, 17 . \mathrm{I} .2013$, Al Ansi et al., 1ex; Wadi Ad Dawaser Nadec Company, 3.X.2012, SN, Al Dryhim, Y., 24exs, on alfalfa; Tabuk: Tabuk-Dhuba Rd., $28^{\circ} 18.39^{\prime} \mathrm{N}, 36^{\circ} 02.87^{\prime} \mathrm{E}, 824 \mathrm{~m}, 15 . \mathrm{IX} .2011$, SN, Al Ansi et al., 6340exs; TabukMadinah Rd., 28 23.47'N, 3651.96'E, 808 m, 14.IX.2011, SN, Al Ansi et al., 1 91 1ex; 14.IX.2011, SN, 11 exs; Qaseem: 1.I.2001, SN, Al Jamhan, 2exs; Sakaka, 2958.15'N, $40^{\circ} 12.18^{\prime} \mathrm{E}, 21 . \mathrm{XI} .2003, \mathrm{SN}, \mathrm{Al}$ Aqal, F., 1 ex.

Local distribution. Specimens of this species are found throughout the kingdom and were collected from Asir, Baha, Eastern province, Makkah, Riyadh, Tabouk, and Qaseem in the present study. This species was previously listed from Saudi Arabia by Martin (1972) and Walker and Pittaway (1987) and reported by Beccari (1971) from Riyadh, Talhouk (1982) from Riyadh and Makkah, and Abdel-Dayem et al. (2017) from Riyadh.

World distribution. E: GR, IT, and ST; N: AG, AZ, EG, LB, and TU; A: AE, AF, ES, GAN, GUI, HEB, IN, IQ, PAL, JO, KI, KU, KZ, MG, NMO, NIN, PA, SA, SCH, SHA, SHX, SI, SY, TR, UP, WS, and XIN (Kováŕ 2007). 


\section{Harmonia Mulsant, 1846}

\section{Harmonia axyridis (Pallas, 1773)}

Coccinella axyridis Pallas, 1773: 716.

Remark. This species is a predator of aphids (Koch 2003).

Material examined. Riyadh: Ad Diriyah, $24^{\circ} 40^{\prime} \mathrm{N}, 46^{\circ} 35^{\prime} \mathrm{E}, 4 . \mathrm{V} .2005, \mathrm{SN}$, Turkestani, S., 1ex.

Local distribution. This species was recently recorded by Biranvand et al. (2019) from Riyadh as a new record to SA.

World distribution. Asia: ES, FE, GAN, GUA, GUI, GUX, HKG, HEB, HEI, HEN, HKG, HUB, HUN, JA, JIA, JIL, JIX, KZ, LIA, MG, NC, NIN, NMO, SC, SCH, SD, SHA, SHN, SHX, TAI, WS, XIN, YUN, and ZHE; introduced to Europe: CZi, FRi, GBi, and GEi; NAR and ORR (Kovár 2007); SA (Biranvand et al. 2019), widely distributed as an invasive species in most continents (Roy et al. 2016).

\section{Hippodamia Chevrolat, 1836}

\section{Hippodamia variegata (Goeze, 1777)}

Adonia variegata Goeze, 1777: 247.

Remark. This is a common species found almost throughout Saudi Arabia in a wide range of habitats. Both larvae and adults of this species are known as predators of aphids.

Material examined. Asir: Abha, Sodah, $1^{\circ} 16.27^{\prime} \mathrm{N}, 4^{\circ} 21.52^{\prime} \mathrm{E}, 24 . \mathrm{IV} .2011$,

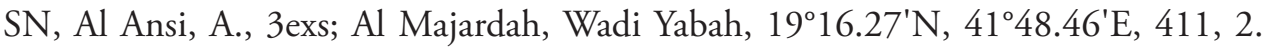
VI.2012, SN, Al Dhafer et al., 1ex; 1.III.2012, LT, 1ex; Khamis Mushait, Wadi Bisha, $18^{\circ} 20.01^{\prime} \mathrm{N}, 42^{\circ} 42.13^{\prime} \mathrm{E}, 1990$ m27.IV.2011, SN, Al Ansi, A., 6exs; Wadi Abha, $18^{\circ} 22.03^{\prime} \mathrm{N}, 42^{\circ} 50.82^{\prime} \mathrm{E}, 1990 \mathrm{~m}$, 28.IV.2011, SN, Al Ansi, A., 15exs; Khamis Mushait, $18^{\circ} 18^{\prime} \mathrm{N}, 42^{\circ} 45^{\prime} \mathrm{E}$, 4.X.2005, SN, Hafedh, A., 1ex; Baha: Wadi Turubah, $20^{\circ} 14.37^{\prime} \mathrm{N}, 41^{\circ} 15.23^{\prime}$ E, 10.V.2011, SN, Fadl, H., 5exs; 14.V.2011, SN, Fadl et al., 14exs; 1.VI.2011, SN, Al Dhafer et al., 1ex; Thee Ain, $19^{\circ} 55.78^{\prime} \mathrm{N}, 41^{\circ} 26.60^{\prime} \mathrm{E}$, 741 m, 11.V.2011, SN, Fadl et al., 11exs; 3.VI.2012, SN, Al Ansi, A., 1ex; 15.V.2011, SN, Fadl et al., 4exs; Wadi Galah, $20^{\circ} 08.08^{\prime} \mathrm{N}, 4^{\circ} 20.56^{\prime} \mathrm{E}, 16 . \mathrm{V} .2011$, SN, Fadl et al., 37exs; Baha Mountain, 25.V.2011, SN, El Hawagry, M., 22exs; Wadi Qanonah, $19^{\circ} 24.67^{\prime} \mathrm{N}, 4^{\circ} 36.39^{\prime} \mathrm{E}, 348 \mathrm{~m}, 11 . \mathrm{V} .2011$, SN, Fadl et al., 15exs; Raghadan, $20^{\circ} 34.25^{\prime} \mathrm{N}, 41^{\circ} 45.11^{\prime} \mathrm{E}, 13 . \mathrm{V} .2011$, SN, Fadl et al., 1ex; Eastern Province: Al Ahsa,

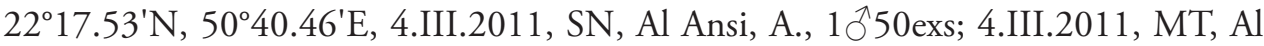
Ansi, A., 4exs; 4.III.2011, SN, Al Ansi, A., 22exs, on alfalfa; An Nuayriyah, $27^{\circ} 25.26^{\prime} \mathrm{N}, 48^{\circ} 27.20^{\prime} \mathrm{E}, 67 \mathrm{~m}, 2 . I I I .2011, \mathrm{SN}, \mathrm{Al}$ Ansi, A., 7exs, on weeds; Qatif, $26^{\circ} 45^{\prime} \mathrm{N}, 49^{\circ} 58^{\prime} \mathrm{E}$, 21.II.1980, 1ex; Hail: Tayma-Hail Rd., $27^{\circ} 43.51^{\prime} \mathrm{N}, 38^{\circ} 12.94^{\prime} \mathrm{E}$, 913 m, 16.IX.2011, SN, Al Ansi, A., 8exs; Makkah: Al Shefa-Taif Rd., 21ํ⒑33'N, $40^{\circ} 23.24^{\prime} \mathrm{E}, 21^{\circ} 08.21^{\prime} \mathrm{N}, 40^{\circ} 21.50^{\prime} \mathrm{E}, 3$. VI.2011, SN, Al Dhafer et al., 2exs; Riyadh: 
Al Ammariah, 244' N, 46²6'E, 18.IV.2007, SN, Ben Jaber, A., 1ex; 14.V.2007, SN, 1ex; Riyadh; 23.I, SN, 1ex; 22.XI.2012, SN, Al Atwi, M., 7exs; 22.XI.2012, SN, Al Agmi, B., 3exs; 29.XII.2008, SN, Abdel-Gayed, A., 2exs; 1.I.2010, SN, Al Ansi, A., 28exs; 11.XI.2009, SN, Al Ansi, A., 6exs; 26.XI.2004, SN, Al Abdulateef, 1ex; 22. XI.2012, SN, Al Wagaan, A., 1ex; 11.XI.2009, SN, Al Shamrani, S., 2exs, on vegetables; 15.III.2011, SN, Setyanengrum, H., 1ex, on weeds; 21.X.2010, SN, Koko, 2exs; 3.V.2011, LT, Koko, 2exs; 22.V.2011, SN, Al Ahmari, S., 6exs; 19.V.2009, SN, Ba Zeiad, A., 1 을 exs; 28.I.2009, SN, 1ex; 18.XI.2009, SN, Setyanengrum, H., $1 \overbrace{}^{\Uparrow} 2$ exs; 13.III.2013, SN, Al Qahtani, F., 5exs; 17.IV.2013, SN, Al Qahtani, F., 2exs; Rawdhet Khoraim, $25^{\circ} 22.98^{\prime} \mathrm{N}, 47^{\circ} 16.71$ 'E, $559 \mathrm{~m}, 201 \mathrm{exs}$ were collected by $\mathrm{SN}$ and B on branches of $A$. ehrenbergiana, A. gerrardii, C. procera L. shawii, R. stricta and Z. nummularia; and PT under canopy of the previous plants; also by MT and LT; through IV.2011; I, III, IV.2012; III.2013; 9.XI.2008, HP, Mutairy, T., 1ex, on weeds; Wadi Namar, $24^{\circ} 32.18^{\prime} \mathrm{N}, 46^{\circ} 34.60^{\prime} \mathrm{E}$, 29.II.2012, HP, Al Ansi, A., $2 \bigcirc^{\top} 3 \mathrm{exs}$, feed on aphids; 6.IV.2012, SN, Al Othman, A., 9exs; Hutet Bani Tamim, 2327.26'N, 46²1.13'E, 8.XI.2010, SN, Al Dhafer et al., 2exs; 28.V.2010, SN, Al Dhafer et al., 1ex, on C. procera; 7.V.2012, SN, Al Ansi, A., 25exs; 4.IV.2008, SN, Al Dhafer et al., 1ex; 15.IV.2010, HP, Al Othman, A., 1ex, on Alfalfa; 10.XI.2011, SN, Al Abdullah, I., 1ex; Al Hariq, 8.V.2012, HP, Abdel-Dayem, M., 1ex; Shuaib Huraymila, 2506.10'N, 4604.22'E, 804 m, 22.IV.2012, HP, Abdel-Dayem, M., 1 ex; 26.V.1993, SN, Asseri, M., 1 ex; Huraymila, 2509' N, 4608'E, 4.VI.1993, SN, Asseri, M., 1 ex; 22.IV.2012,

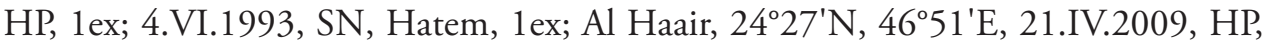
Al Ansi, A., 2exs; 13.V.2009, SN, Al Ansi, A., 3exs; 1.II.2009, SN, Al Harbi, S., 1ex; Sajir, $4^{\circ} 51.23^{\prime} \mathrm{N}, 45^{\circ} 42.39^{\prime} \mathrm{E}, 17 . X I .2011$, SN, Setyanengrum, H., 5exs; Al Muzahmiyah Al Khararah, $24^{\circ} 24.21^{\prime} \mathrm{N}, 46^{\circ} 14.40^{\prime} \mathrm{E}, 29 \mathrm{exs}$ were collected by SN and LT; III-V, VIII, X.2011; IV.2012; 31.III.1993, SN, Al Saleh, 1ex, on weeds; Al Uyaynah, $24^{\circ} 53^{\prime} \mathrm{N}, 46^{\circ} 22^{\prime} \mathrm{E}, 27 . I I .2013$, SN, Al Qahtani, F., 5ex; 13.III.2013, SN, Al Qahtani, F., 4exs; 15.XII.2010, SN, Al Abdul Muhsen, 2exs; 30.XI.2010, SN, Al Ansi, A., 1ex, on alfalfa; 3.V.2011, SN, Al Ahmari, A., 3exs; 25.VI.2011, SN, Al Hazmy, 3exs; 13.III.2011, SN, Al Shehri, 1ex; 14.V.2011, SN, Al Hazmy, 1ex; 9.V.2011, LT, Al Ahmari, A., 1ex; 23.III.2011, SN, Al Ahmari, A., 3exs; 22.V.2011, SN, Al Ahmari, A., 2exs; 28.IV.2010, SN, Al Hashel, A., 4exs; 7.IV.2010, SN, Al Dawsari, M., 1ex; 10.III.2010, SN, Al Hashel, A., 1ex; 10.III.2010, SN, Al Haori, M., 5exs; 10.III.2010, SN, Al Mutairy, A., 3exs; 10.III.2010, SN, Al Othman, A., 6exs; 10.III.2010, SN, Al Otaibi, A., 4exs; 12.V.2010, SN, Al Hashel, A., 1ex; 10.III.2010, SN, Al Salem, B., 1ex; 7.IV.2010, SN, Al Mutairy, A., 1ex; 28.IV.2010, SN, Al Otaibi, A., 2exs; 10. III.2010, SN, Al Hazazi, T., 2exs; 28.IV.2010, SN, Al Othman, A., 1ex; 27.II.2008, SN, Al Amodi, 2exs; 14.III.2007, SN, Al Ahmadi, M., 3exs; 7.IV.2010, SN, Al Yousif, A., 2exs; 27.II.2008, SN, Al Tork, 2exs; 7.V.2008, SN, Al Tork, 2exs; 9.IV.2008, SN, Al Sharani, 2exs, on alfalfa; 28.IV.2009, SN, Al Ansi, A., 3exs; 30.XI.2010, SN, Al Ansi, A., 3exs; 12.V.2010, SN, Al Yousif, A., 3exs; 7.IV.2010, SN, Al Salem, B., 1ex; 24.II.2010, SN, Al Yousif, A., 1 ex; 10.III.2010, SN, Al Dawsari, M., 1ex; 28.IV.2010, SN, Al Yousif, A., 1ex; 15.II.2010, SN, Al Yousif, A., 3exs; 15.XII.2010, SN, Al Harbi, S., 2exs; 3.XI.2010, SN, Al Ghunaim, A., 1ex; 15.XII.2010, SN, Al Dhabani, L., 
1ex; 1.XI.2010, SN, Al Ghunaim, A., 1ex; 12.X.2011, SN, Al Omar, 2exs; 28. IX.2011, SN, Al Omar, 1ex; 13.III.2007, SN, Al Raqbah, R., 1ex; 12.X.2005, SN, Al Yami, M., 1ex; 4.VI.1993, SN, 2exs, on weeds; 6.V.1993, SN, 2exs, on weeds; 2. IV.1993, SN, 2exs, on weeds; 8.VI.1993, SN, 2exs, on weeds; 4.V.1993, SN, 1 ex, on weeds; 25.V.1993, SN, 1ex, on weeds; 25.II.2004, SN, Hatem, 1ex, on alfalfa; 7. III.2012, SN, Al Edwani, M., 5exs; 4.IV.2012, SN, Al Ghamedi, R., 1ex; 8.II.2012, SN, Al Ghamedi, R., 4exs; 7.III.2012, SN, Al Ghamedi, R., 10exs; 8.II.2012, SN, Al Qahtani, R., 14exs; 4.IV.2012, SN, Al Wagaan, A., 2exs; 7.III.2012, SN, Al Atwi, M., 4exs; 8.II.2012, SN, Al Wagaan, A., 4exs; 8.II.2012, SN, Al Agmi, B., 2exs; 7. III.2012, SN, Al Agmi, B., 2exs; 4.IV.2012, SN, Al Agmi, B., 1ex; Ben Jabr, 15.V.2011, LT, Al Shahri, A., 2exs; Al Waseel, $24^{\circ} 48.40^{\prime} \mathrm{N}, 46^{\circ} 30.42^{\prime} \mathrm{E}, 12 . X I .2008$, SN, Ba Zeiad, A., 1ð, on alfalfa; 5.V.2004, SN, 1ex, on alfalfa; 13.IV.1993, SN, 1ex; 14.III.2012, SN, Al Atwi, M., 5exs; 14.III.2012, SN, Al Wagaan, A., 1ex; 14.III.2012, SN, Al Edwani, M., 2exs; 21.XI.2009, SN, Al Ansi, A., 13exs; 21.XI.2009, SN, Single, A., 2exs; 20.X.2011, SN, Al Matter, E., 1 ex; 20.X.2011, SN, Al Rashedi, 1ex; 20.XI.2011, SN, Al Matter, E., 4exs; 26.V.2010, SN, Al Yousif, A., 1ex; 26.V.2010, SN, Al Othman, A., 1ex; 1.XII.2010, SN, Al Dhahbani, L., 1ex; 11.V.2010, SN, Husain, M., 1ex; Wadi Laban, $24^{\circ} 38.39^{\prime} \mathrm{N}, 46^{\circ} 37.20^{\prime} \mathrm{E}, 21 . X I .1990$, SN, Al Mutlaq, 1ex, on vegetables; Riyadh, 6.XII.2006, ST, 1ex; Nesah, $24^{\circ} 43.32^{\prime} \mathrm{N}, 46^{\circ} 41.12^{\prime} \mathrm{E}, 30 . \mathrm{XI} .2005$, SN, 1ex; Al-Quway'iyah, $24^{\circ} 20^{\prime} \mathrm{N}, 4^{\circ} 09^{\prime} \mathrm{E}, 18 . V .2012$, SN, Al Ghamedi, R., 12exs; Tumayer, 2542.36'N, 4552.11'E, 9.V.2011, LT, Al Ahmari, S., 9exs; 10.II.2010, MT, Al Dhafer, H., 1ex; 25.I.2010, MT, Al Dhafer, H., 1ex; Al Kharj, Ar Rafai, $20^{\circ} 24^{\prime} \mathrm{N}, 4^{\circ} 29^{\prime} \mathrm{E}$, 4.IV.2007, SN, Shraf Addeen, A., 9exs, on alfalfa; 16.V.2007, SN, Al Grid, S., 4exs; 4.IV.2007, SN, Al Shehri, 3exs; Al Kharj, 20²4'N, 46 $29^{\circ} \mathrm{E}$, 14.XI.2007, SN, Al Salman, 3exs; 26.XI.2008, SN, Valenza, B., 1ex; 1.IV.2009, SN, Al Zahrani, M., 2exs; 23.XII.2008, SN, Setyanengrum, H., 1ex, on vegetables; 24.III.2010, SN, Al Haori, M., 2exs; 24.III.2010, SN, Al Dawsari, M., 1ex; 24. III.2010, SN, Al Mutairy, A., 1ex; 9.III.2011, SN, Al Hazmy, H., 1ex; 1.IV.2009, SN, Al Amri, A., 17exs; 2.V.2007, SN, 3exs; 1.IV.2009, SN, Al Sebiai, M., 1ex; 2005, SN, 2exs; Ad Diriyah, $24^{\circ} 40^{\prime} \mathrm{N}, 46^{\circ} 35^{\prime} \mathrm{E}, 23 . X I .2011$, LT, Al Kassem, A., 1ex; 23.X.1989, SN, 1ex; 8.XII, SN, 1ex, on alfalfa; 18.III.2009, SN, Al Sebiai, M., 4exs, on alfalfa; 29.X.2008, SN, Al Ahmari, A., 1ex, on alfalfa; 11.III.2009, SN, Al Harbi, M., 5exs; 2.VI.2009, SN, Al Zahrani, M., 1ex; 29.X.2008, SN, Ba Zeiad, A., 2exs, on alfalfa; 5.XII.2010, SN, Abu Al Raha, W., 2exs; 8.X.2000, SN, Hatan, 2exs; 14.X.2003, SN, Al Dhubaib, 1ex; 9.X, SN, Mustafa, 1ex; 15.X.2003, SN, Al Yaqoob, 1ex; 14.X.2003, SN, Al Saiary, 1ex; 26.IX.2011, SN, Al Omar, 1ex; 1.XII.2010, SN, Abdel Gayed, A., 1ex; 29.X.2008, SN, Al Qahtani, M., 1ex, on alfalfa; 23.XI.2011, LT, Al Kassem, A., 1ex; 23.IV.2009, SN, Husain, M., 1ex; 14.V.2009, SN, Sofan, A., 1ex; 7.IV.2010, SN, Al Salem, B., 1ex; 7.IV.2010, SN, Hazazi, T., 1ex; 17.III, SN, 5exs, on vegetables; 24.III, SN, 10exs, on vegetables; 22.III, SN, 1ex, on vegetables; 15.X.2000, SN, Al Khurigi, M., 1ex; 22.XI.1990, SN, Asseri, M., 1ex, on alfalfa; 25.IX.2005, SN, 1ex, on alfalfa; 1.XI, SN, Al Qarni, A., 3exs; 20.X.1993, SN, Al Aseri, 2exs, on alfalfa; 5. VIII, SN, Al Qarni, A., 1ex; 17.III.1989, SN, 1ex, on barley; 16.X.1989, SN, 1ex, on alfalfa; 15.IV. SN, Adel, 1ex, on vegetables; 20.XI.1991, SN, Ghazi, 1ex, on alfalfa; 
30.X.1989, SN, 1ex, on barley; 29.III.1989, SN, 4exs, on Barley; 14.IV.1993, SN, Aziz, A., 4exs, on Alfalfa; XI.1989, SN, 1ex, on alfalfa; V.1986, SN, 1ex, on alfalfa; 14.XII.1992, SN, 193 exs, on alfalfa; 22.XI.1991, SN, Ghazi, 1ex; 17.V.1989, SN, 1ex; 7.XI.1990, SN, Al Qarni, A., 2exs, on vegetables; 20.IV.1992, SN, Saif, A., 2exs, on alfalfa; 26.IV.1992, SN, Saif, A., 2exs; 9.IV.2003, SN, Al Habeeb, A., 1ex; 5. VI.1993, SN, Aziz, A., 1ex; 25.IX.2005, SN, 1ex; 21.IV, SN, Nabeel, 1ex; 30.X.1989, SN, 1ex, on alfalfa; 4.XII.1992, SN, 1ex, on alfalfa; 30.XII.1989, SN, Amro, 1ex, on vegetables; 7.XI, SN, Al Jaber, 1ex, on vegetables; Education Farm, $24^{\circ} 40^{\prime} \mathrm{N}, 46^{\circ} 35^{\prime} \mathrm{E}$, 18.V.2013, SN, Al Qahtani, F., 1ex; 25.X.2009, SN, Al Ahmari,S., 1ex, on alfalfa; Ad Diriyah Erqah, 11.II, SN, Al Issa, M., 2exs; 16.XI.1989, SN, 1ex, on alfalfa; 21.XI, SN, Al Jaberi, 2exs, on vegetables; 10.III.1987, SN, 1ex, on alfalfa; 12.IV.1993, SN, 1ex; 9.I, SN, 1ex, on alfalfa; 18.V.1993, SN, 2exs, on alfalfa; 4.V.1978, SN, 1ex, on alfalfa; 9.V, SN, Moshal, 1ex, on alfalfa; 17.III.2004, SN, Al Hugail, Y., 1ex; 12. III.2003, SN, Al Awadhi, A., 1ex, on alfalfa; 24.III.2003, SN, Al Harbi, 1ex, on alfalfa; 12.III.2003, SN, Al Habeeb, A., 1ex, on alfalfa; 4.III, SN, 1ex, alfalfa; 9.I, SN, 1ex, on alfalfa; 16.III.2005, SN, 1ex; 4.VIII, SN, 1ex; 17.V.2011, SN, Al Etwy, Y., 1ex; 23.X.1992, SN, 1ex, on alfalfa; 30.XII.2009, SN, Sofan, A., 2exs; 21.IV.1987, SN, 2exs, on alfalfa; 5.V.1987, SN, 1ex, on alfalfa; 4.V.1987, SN, 1ex, on alfalfa; 8. XI.1989, SN, 1ex, on alfalfa; 12.IV.1993, SN, 1ex, on alfalfa; 23.IV, SN, 1ex, on alfalfa; 7.IV.1987, SN, 11 exs, on alfalfa; 15.XI.2006, SN, Al Qahtani, A., 2exs, on alfalfa; 19.V.2009, SN, Al Motaeb, M., 9exs, on alfalfa; 2.X.1985, SN, 1ex, on alfalfa; 15.XI.2006, SN, Al Dawood, A., 1ex; 16.V.2007, SN, Shraf Addeen, A., 1ex, on alfalfa; 8.VI.2009, SN, Belhareth, H., 1ex, on alfalfa; 2.VI.2009, SN, Al Ghamedi, R., 1ex; 25.VII.1998, SN, Al Qarni, A., 1ex; 5.I.1687, SN, Amro, 3exs, on alfalfa; 14.III.2010, ST, Al Dhafer, H.; El Gharabawy, A. and El Torkey, A., 1ex; 10.IV.2001, SN; 5.VII, SN, Al Qarni, A., 1ex; 29.XII.1986, SN, 1ex, on alfalfa; 19.I.1987, SN, Amro, 2exs, on weeds; 12.III.2003, SN, Al Shathri, 1ex; 12.I.1987, SN, 5exs; 31. III.1987, SN, 3exs; 12.IV.1993, SN, 1ex; 24.XI.1986, SN, 1ex;17.X.2011, SN, Al Khudairi, H., 1ex; 2.V.2010, SN, Al Dhafer et al., 4exs; 30.XII.2009, SN, Sofan, A., 1ex;19.XII.2010, SN, Al Ansi, A., 1ex, on alfalfa; 31.X.2010, SN, Al Ansi, A., 2exs, on alfalfa; 7.III.2011, SN, Abdel-Gayed, 4exs, on fig plant; 28.III.2010, PT, Al Dhafer et al.,1ex; 10.III.1987, SN, 1ex; 24.III.1987, SN, 1ex; 5.V.1987, SN, 1ex; Wadi Ad Dawaser Nadec Company, 3.X.2012, SN, Al Dryhim, Y., 8exs, on alfalfa; Riyadh, 11.IX.1969, SN, R. D. Pope, ANMA, 2exs, feed on aphids; same location 21.IV.1980, SN, Talhouk et al., ANMA, 16exs; same data except 13.V.1980, SN, ANMA, 8exs; Tabuk: Tabuk-Dhuba Rd., $28^{\circ} 18.39^{\prime} \mathrm{N}, 36^{\circ} 02.87^{\prime} \mathrm{E}, 824 \mathrm{~m}$, 15.IX.2011, SN, Al Ansi, A., 1ठ2o; 15.IX.2011, SN, Al Ansi, A., 3exs; 15.IX.2011, SN, Al Ansi, A., 28exs; 15.IX.2011, SN, Al Ansi, A. 155exs, (preserved in alcohol); Tabuk-Madinah Rd., 28 23.47'N, 36'51.96'E, 808 m, 14.IX.2011, SN, Al Ansi, A., 10exs; 14. IX.2011, SN, Al Ansi, A., 8exs; 14.IX.2011, SN, Al Ansi, A., 1ex; 14.IX.2011, SN, Al Ansi, A., 4exs; 14.IX.2011, SN, Al Ansi, A., 1ex.

Local distribution. Asir, Baha, Eastern Province, Makkah, Riyadh, and Tabuk. It was previously recorded from Jizan by Beccari (1971), from Riyadh and Makkah by 
Talhouk (1982) and by El-Hawagry et al. (2013), from Baha and Abdel-Dayem et al. (2017) from Riyadh and listed by Walker and Pittaway (1987).

World distribution. Asia: AE, AF, BEI, BT, FE, FUJ, GAN, HEB, HEN, HP, HUN, IN, IQ, PAL, JIL, JO, KA, KI, KZ, LE, LIA, MG, NC, NMO, NIN, NP, PA, SA, SC, SCH, SHA, SHX, SD, SI, SY, TD, TM, TR, UP, UZ, WS, YE, YUN, XIN, and XIZ; Europe: $\mathrm{AB}, \mathrm{AE}, \mathrm{AL}, \mathrm{AN}, \mathrm{AR}, \mathrm{AU}, \mathrm{BE}, \mathrm{BH}, \mathrm{BU}, \mathrm{BY}, \mathrm{CR}, \mathrm{CT}, \mathrm{CZ}, \mathrm{DE}$, EN, FI, FR, GB, GE, GG, GR, HU, IT, LA, LS, LT, LU, MC, MD, NL, NT, PL, PT, RO, SK, SL, SP, ST, SV, SZ, TR, UK, and YU; North Africa: AG, CI, EG, LB, MO, MR, and TU; AFR, NAR, and ORR (Kovár 2007).

\section{Oenopia Mulsant, 1850}

\section{Oenopia oncina (Olivier, 1808)}

Coccinella oncina A. G. Olivier, 1808: 1048.

Remark. Presence of this species could not be confirmed, although it was listed from the KSA by Kovár (2007).

World distribution. Asia: AE, AF, GAN, IN, IQ, PAL, JO, KA, KI, KU, KZ, LE, MG, PA, SA, SI, SY, TD, TM, TR, and UZ; Europe: AB, AR, BU, GR, and TR; North Africa: EG (Kovár 2007).

\section{Psyllobora Chevrolat, 1836}

\section{Psyllobora bisoctonotata (Mulsant, 1850)}

Thea bisoctonotata Mulsant, 1850: 204.

Remarks. This species was found in a variety of habitats from greenhouses, open crop fields, and in natural habitats from low to highlands. Both adults and larvae of this species feed on fungi (Raimundo and van Harten 2000).

Material examined. Asir: 8-20 km outside from Abha-Taif Rd., 1976, Wittmer et al., ANMA, 1ex; Abha, Sodah, $18^{\circ} 16.27^{\prime} \mathrm{N}, 42^{\circ} 21.52^{\prime} \mathrm{E}$, 19.III.2009, SN, Al Dhafer et al., $5 \hat{2} 2$, on squash plants; 24.IV.2011 SN, Al Ansi et al., 2犬̂; Abha, Raydah, $18^{\circ} 12.10^{\prime} \mathrm{N}, 42^{\circ} 24.54^{\prime} \mathrm{E}, 2578$ m, 8.II.2016, SU, Al Ansi, A., 1ex; Ahd Rifidh, $18^{\circ} 06.33^{\prime} \mathrm{N}, 42^{\circ} 53.82^{\prime} \mathrm{E}, 16.1 .2013 \mathrm{BS}$, Al Ansi et al., 7우 $0^{\top}$; Baha: Thee Ain, $19^{\circ} 55.78^{\prime} \mathrm{N}, 41^{\circ} 26.60^{\prime} \mathrm{E}, 741 \mathrm{~m}, 15 . \mathrm{V} .2011$, SN, Fadl et al., 10'; 13.X.2010, SN, Al Dhafer et al., 10; 12.IV.2016, SU, Al Ansi, A., 2exs; Wadi Turubah, 20¹4.37'N,

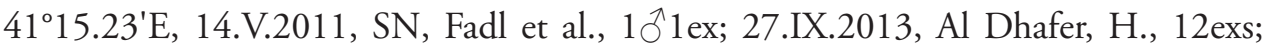
Al Atawlah, $20^{\circ} 18.37^{\prime} \mathrm{N}, 41^{\circ} 20.52^{\prime} \mathrm{E}, 2160 \mathrm{~m}$, 24.IV.2013, BS, Al Ansi et al., 11 exs; Makkah: Taif, $21^{\circ} 08^{\prime} \mathrm{N}, 40^{\circ} 58^{\prime} \mathrm{E}, 10 . \mathrm{IX} .1969$, SN, ANMA, 4exs; Najran: Rijila, Wadi Najran, $17^{\circ} 31.56^{\prime} \mathrm{N}, 44^{\circ} 13.65^{\prime} \mathrm{E}, 1257 \mathrm{~m}$, 15.I.2013, BS, Al Ansi et al., $20{ }^{\top} 8$ \% 15.I.2013, SN, Al Ansi et al., 1ठ'; Wadi Shuaib Barran, $17^{\circ} 28.94^{\prime} \mathrm{N}, 4^{\circ} 05.52^{\prime} \mathrm{E}$, 
1325 m, 16.I.2013, BS, Al Ansi et al., 1ðð; Riyadh: Ad Diraiyah, 5.VIII.1983, Abu Thoria, ANMA, 1ex; 21.XII.1963, SN, Abu Thoria, ANMA, 1ex.

Local distribution. This species is widely distributed in the KSA and was collected from Asir, Baha, Makkah, Najran, and Riyadh. It was previously reported by Shalaby (1962) from Makkah, Beccari (1971) from Makkah, Fürsch (1979) from Asir, and Talhouk (1982) from Makkah.

World distribution. Asia: AE, AF, IN, IQ, PAL, JO, KA, PA, SA, SY, UP, and YE; North Africa: EG; AFR and ORR (Kovár 2007).

Xanthadalia Crotch, 1874

Xanthadalia effusa rufescens (Mulsant, 1850)

Xanthadalia effusa (Erichson, 1843)

Harmonia rufescens Mulsant, 1850: 76.

Remark. This is a rare species, limited to only Afrotropical region; its presence in the KSA was not confirmed, and only Kovár (2007) enlisted it from the country.

World distribution. Asia: SA and YE; North Africa: EG; AFR (Kovár 2007).

\section{Epilachnini Mulsant, 1846}

Henosepilachna Li, 1961

\section{Henosepilachna birta (Thunberg, 1781)}

Coccinella hirta Thunberg, 1781: 23.

Remark. This species was found from high elevated lands of Asir province and exhibited an Afrotropical distribution. It is a phytophagous coccinellid.

Material examined. Asir: Abha, $18^{\circ} 14^{\prime} \mathrm{N}, 42^{\circ} 31^{\prime} \mathrm{E}, 27-28$. VIII.1978, Shalaby, F., ANMA, 2exs; Abha Raydah, 18¹2.10'N, 42²4.54'E, 2578 m, 4.XI.2013, HP, Abdel-Dayem et al., 1; 8.II.2016, SU, Al Ansi, A., 35ํ.

Local distribution. Specimens of this species were collected from Raydah mountains of Asir and previously reported by Shalaby (1962) from Asir and by Beccari (1971) from Asir.

World distribution. Asia: SA (Shalaby 1962; Beccari 1971); South, West, and Central Africa (Fürsch 1991).

\section{Chnootriba Chevrolat, 1837}

Chnootriba elaterii orientalis (Zimmermann, 1936)

Coccinella elaterii Rossi, 1794

Coccinella chrysomelina Fabricius, 1775 (1): 277. 
Remark. This is a phytophagous coccinellid. Members of this species were found to be associated with squash and cucurbits. This species is phytophagous and has been reported as a serious pest of Cucurbitaceae (Raimundo and van Harten 2000).

Material examined. Asir: Khamis Mushait Wadi Bin Hashbal, $18^{\circ} 35.44^{\prime} \mathrm{N}$, 4239.01'E, 1892 m, 26.IV.2011, SN, Al Ansi, A., 1ex; Khamis Mushait, $18^{\circ} 18^{\prime} \mathrm{N}$, $42^{\circ} 45^{\prime} \mathrm{E}$, 17.IV.2016, SN, Soliman, A., 1ex; Abha Raydah, $18^{\circ} 12.10^{\prime} \mathrm{N}, 42^{\circ} 24.54^{\prime} \mathrm{E}$, 2578 m,5.XII.2013, HP, Khan, S., 1ex; 16.IV.2016, HP, Al Dhafer, H., 1ex; Thalooth Al Mandhar Wadi Baqrah, 1847.57'N, 4201.12'E, 433 m, 12.X.2013, HP, El Torkey, A., 6exs; HP, Rasool, I., 2exs; Baha: Wadi Ghanuna, 19²4.67'N, 41'36.39'E,

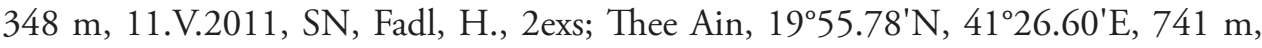
11.V.2011, SN, Fadl, H., 1ex; 11.IV.2016, SN, Al Ansi, A., 1ex; Wadi Al Arg QilwahAdhom Rd., 20²9.98'N, 4048.95'E, 429 m, 9.XI.2012, BS, Fadl, H., $10^{\top} 1$ ex; Jizan: Baysh, $17^{\circ} 42.71^{\prime} \mathrm{N}, 42^{\circ} 53.19^{\prime} \mathrm{E}, 102 \mathrm{~m}, 23 . I I .2015$, HP, Al Harbi et al., 1ex; Wadi Gowra Aiban, $17^{\circ} 17.57^{\prime} \mathrm{N}, 43^{\circ} 04.21^{\prime} \mathrm{E}, 451 \mathrm{~m}, 11 . X I .2012$, SN, Fadl, H., 1ex; Jizan city, $16^{\circ} 54^{\prime} \mathrm{N}, 42^{\circ} 29^{\prime} \mathrm{E}, 20 . \mathrm{I} .1979$, SN, Talhouk et al., ANMA, 12exs, det. Fürsch, 1979. Makkah: Al-Taif, $21^{\circ} 08^{\prime} \mathrm{N}, 40^{\circ} 58^{\prime} \mathrm{E}, 19 . \mathrm{II} .1980$, 2exs; Riyadh: Hutet Bani Tamim, $23^{\circ} 27.26^{\prime} \mathrm{N}, 46^{\circ} 41.13^{\prime} \mathrm{E}, 30 . X I I .2010$, HP, Al Dryhim et al., 2exs; 2.VI.2007, SN, Al Dryhim et al., 1ex; Huraymala, 27.VI.1988, SN, Al Dawood, A., 13exs, on

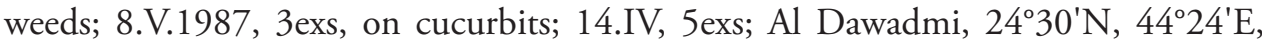
22.II.1980, 1ex; Dirab, $2^{\circ} 30^{\prime} \mathrm{N}, 46^{\circ} 51^{\prime} \mathrm{E}, 17 . X I .1989$, 1ex; 17.XI.1986. Amro, 1ex, on lantana; 17.XI, SN, Al Fehaid, M.,1ex; Al-Hair, 13.I.1989, Salem, 9exs, on colocynth; Al Kharj, $20^{\circ} 24^{\prime} \mathrm{N}, 46^{\circ} 29^{\prime} \mathrm{E}, 31 . X .2002$, Saif, A., 1ex, on Squash; Tumayer, $25^{\circ} 42.36^{\prime} \mathrm{N}, 4^{\circ} 52.11^{\prime} \mathrm{E}, 9 . V .2011$, LT, Al Ahmari, S., 1ex; Riyadh, 2.XI.2013, HP,

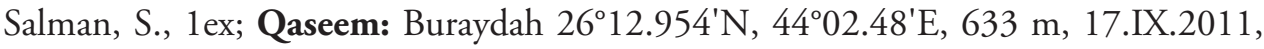
$\mathrm{SN}, \mathrm{Al}$ ansi et al., $1 \mathrm{ex}$.

Local distribution. This species is widely distributed in Saudi Arabia and was collected from Asir, Bahah, Jizan, Makkah, Riyadh, and Qaseem. It was previously recorded from Zilfi approximately 160 miles northwest of Riyadh by Kapur (1959); from Eastern Province, Riyadh, Makkah, and Tabouk by Beccari (1971); and from Riyadh by Fürsch (1979) and listed by Martin (1972) and Walker and Pittaway (1987).

World distribution. Asia: AF, CY, IN, IQ, PAL, JO, SI, SA, SY, PA, TR, and YE; North Africa: EG and LB; AFR (Kovár 2007).

\section{Noviini Mulsant, 1850}

\section{Novius Mulsant, 1850}

Remark. The species of genus Rodolia have been placed in Novius based on the latest paper by Pang et al. (2020).

\section{Novius argodi (Sicard, 1909)}

Rodolia argodi Sicard, 1909: 142. 
Remark. This species is known as a predator of scale insects, including Icerya purchasi Maskell, as mentioned in the label data by Fürsch (1968); it is a pest of Citrus spp. and Casuarina sp. (Martin 1972).

Material examined. Riyadh: Specimen was taken from ANMA collection that was collected from Riyadh city, V.1966, ANMA, 1ex, det. Fürsch 1968.

Local distribution. This species was listed by Martin (1972) and reported from Asir and Makkah by Abu-Thuraya (1982).

World distribution. Asia: SA (Martin 1972; Abu-Thuraya 1982); it is widespread in Africa (Raimundo and van Harten 2000); AE (Raimundo et al. 2007).

\section{Novius cardinalis (Mulsant, 1850)}

Vedalia cardinalis Mulsant, 1850: 906.

Remark. This species became famous at the end of the $19^{\text {th }}$ century when it was introduced from its native country Australia to California for the control of cottony cushion scale (I. purchasi) in citrus orchards, producing the first major success in biological control. This species is now introduced to all continents, except Antarctica (Raimundo and van Harten 2000).

Material examined. Asir: Khamis Mushait, Wadi Baisha, 27.IV.2011, SN, Al Ansi, A., 1 ; ; Baha: Al Mandaq, Wadi Turubah, $20^{\circ} 14.37^{\prime} \mathrm{N}, 41^{\circ} 15.23^{\prime} \mathrm{E}, 3 . \mathrm{VI} .2012$, BS, Al

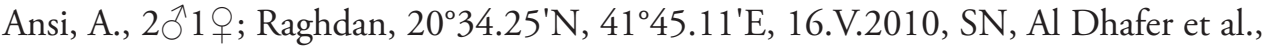
1 ; ; Rahwan, $20^{\circ} 04.03^{\prime} \mathrm{N}, 4^{\circ} 27.01^{\prime} \mathrm{E}, 2272 \mathrm{~m}, 24 . \mathrm{V} .2013$, BS, Al Ansi et al., 2exs; King Saud Rd., 2000.28'N, 41 27.91'E, 2132 m, 25.V.2013, BS, Al Ansi et al., 3exs.

Local distribution. Specimens of this species were collected from Asir and Baha provinces and previously reported from Najran by Abu-Thuraya (1982).

World distribution. Asia: AE, FUG, HAI, GUA, HEN, HKG, HUB, IN, PAL, JA, JIA, JIX, JO, PA, SC, SCH, SHA, SHG, TAI, TR, YUN, and ZHE; Europe: AL, BU, CR, FR, GR, IT, MA, PT, SP, and TR; North Africa: CI, AG, EG, LB, MO, and TU; AFR, AUR, NAR, NTR, and ORR (Kovár 2007).

\section{Novius yemenensis (Raimundo \& Fürsch, 2006)*}

Figure 3

Rodolia yemenensis Raimundo \& Fürsch, 2006: 219-220.

Diagnosis. This species is restricted to the Arabian Peninsula and can be identified by the following characters: body entirely reddish to reddish brown, some specimens have blackish head and pronotum, without black patterns on elytra and with white semierect short setae. Punctures on elytra are larger than those on pronotum. Base of elytra is slightly concave with rounded shoulders. 


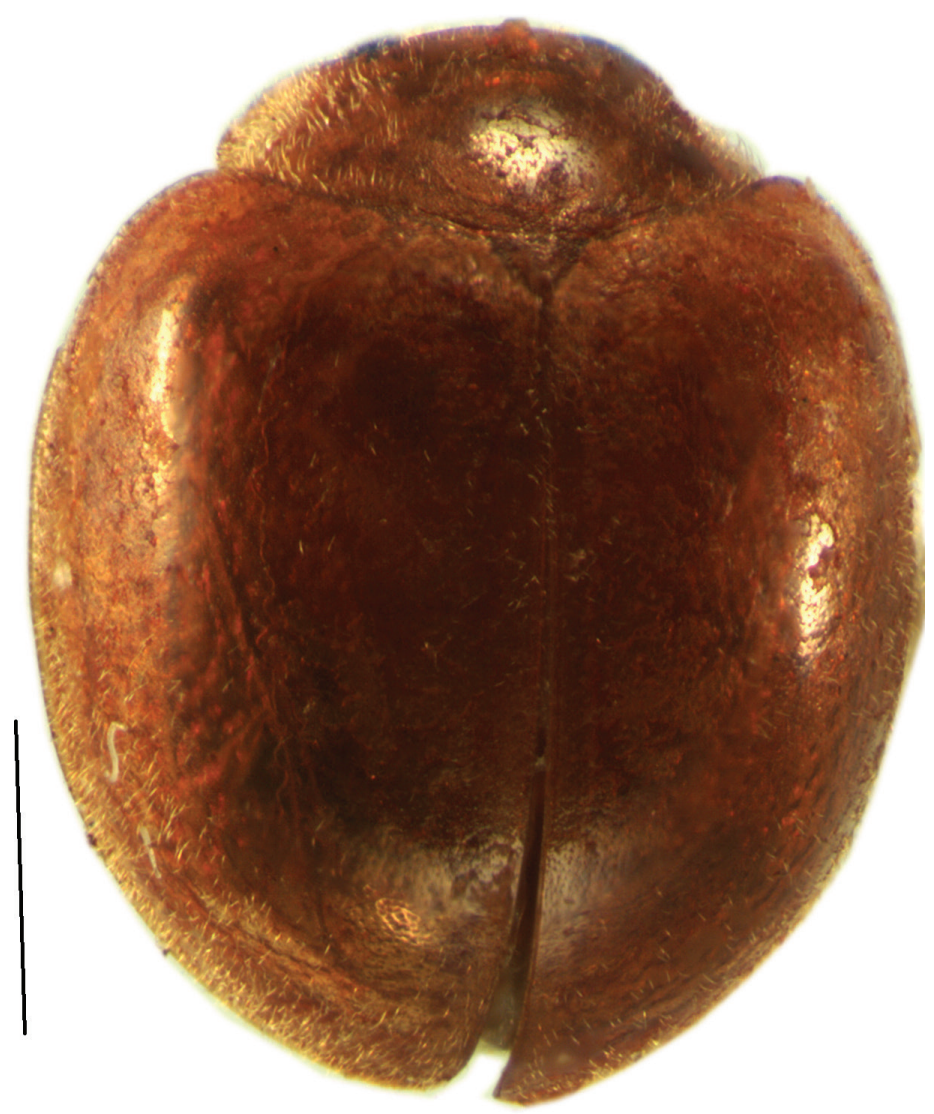

Figure 3. Dorsal view of Novius yemenensis. Scale bar: $1 \mathrm{~mm}$.

Material examined. Asir: Thalooth Al Mandhar Wadi Baqrah, $18^{\circ} 47.57^{\prime} \mathrm{N}$, 4201.12'E, 433 m, 12.X.2013, HP, Abdel-Dayem, M., 19; Rijal Almaa, Wadi Sabin, $17^{\circ} 48.25^{\prime} \mathrm{N}, 4^{\circ} 21.64^{\prime} \mathrm{E}, 194 \mathrm{~m}$, 10.II.2016, SU, Al Ansi, A., 19; Jizan: Sabya-

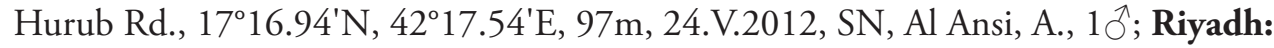
El-Jola Riyadh-Makkah Rd., $2^{\circ} 20.42^{\prime} \mathrm{N}, 45^{\circ} 47.29^{\prime} \mathrm{E}, 11 . X .2010$, SN, Al Dhafer et al., 10'; King Abdullah street, in front of King Abdulaziz city, 2443.01 'N, 4638.65 'E, 21.III.2010, HP, El Gharbawy, A., $10^{\top}$; Dirab, $23^{\circ} 30^{\prime} \mathrm{N}, 46^{\circ} 51^{\prime} \mathrm{E}, 1 . \mathrm{XI} .2009$, ST, Al Dhafer et al., 1 ㅇ․

Local distribution. This species was collected from Asir, Jizan, and Riyadh provinces. World distribution. Asia: YE (Raimundo et al. 2006); newly recorded from SA.

\section{Diomus Mulsant, 1850}

\section{Diomus rubidus (Motschulsky, 1837)}

Scymnus rubidus Motschulsky, 1837: 418. 
Material examined. Riyadh: As Sullayyil-Najran Rd., $18^{\circ} 01.35^{\prime} \mathrm{N}, 42^{\circ} 45.81^{\prime} \mathrm{E}$, 619 m, 17.I.2013, BS, Al Ansi, A., 10’; Rawdhet Khoraim, 2522.98'N, 47²16.71'E, 559 m, 14.IV.2012, PT, $1 \overbrace{}^{\lambda} 1$, under C. procera, 14.IV.2012, PT, $1 \delta^{\Uparrow} 1$, under $L$. Shawii; 23.VI.2012, PT, 1 q, under C. procera.

Local distribution. It was collected from Riyadh (Abdel-Dayem et al. 2017) and previously reported by Fürsch (1979) from Riyadh.

World distribution. Asia: IN, IQ, PAL, LE, SA, SY, and YE; Europe: AR, CR, FR, GR, and IT; North Africa: AG, EG, LB, MO, and TU (Kovár 2007); AE (Raimundo et al. 2007).

\section{Hyperaspidini Mulsant, 1846}

Hyperaspis Chevrolat, 1836

Hyperaspis polita Weise, 1855

Hyperaspis polita Weise, 1855: 60.

Remark. This species is a predator of the mealybug Nipaecoccus viridis (Newstead, 1894) (Alizadeh et al. 2013).

Materials examined. Riyadh, 2.II.1980, Talhouk et al., ANMA, 1 ㅇ․

Local distribution. It was collected from Riyadh and previously listed in the taxonomic key by Fürsch (1979).

World distribution. Asia: AF, CY, PAL, SY, TD, TM, and TR; North Africa: LB; AFR (Kovár 2007), SA (Fürsch 1979).

\section{Hyperaspis pumila pumila Mulsant, 1850}

Hyperaspis pumila Mulsant, 1850: 655.

Material examined. Asir: Thee Ain, $19^{\circ} 55.78^{\prime} \mathrm{N}, 41^{\circ} 26.60^{\prime} \mathrm{E}, 741 \mathrm{~m}, 15 . \mathrm{V} .2011$, SN, Fadl et al., 19; Thalooth Al Mandhar Wadi Baqrah, $18^{\circ} 47.57^{\prime} \mathrm{N}, 42^{\circ} 01.12^{\prime} \mathrm{E}, 433 \mathrm{~m}$, 4.XI.2013, LT, Al Dhafer et al., 1 9 ; Muhail Wadi Hali, $18^{\circ} 30.12^{\prime} \mathrm{N}, 42^{\circ} 02.21^{\prime} \mathrm{E}$, 440 m, 11.II.2016, SU, Al Ansi, A., 19; Dirab, 2330'N, 4651'E, 19.III.2014, PT, Al Harbi, M., 1 , under palm tree.

Local distribution. This species was collected from Asir, Baha, and Riyadh provinces. It was previously catalogued only by Kovár (2007) with no information about its distribution in the KSA.

World distribution. Asia: SA and YE; North Africa: LB; AFR (Kovár 2007).

Hyperaspis vinciguerrae Capra, 1929

Hyperaspis vinciguarae Capra, 1929: 241. 
Remark. This species is a predator of aphids (Raimundo et al. 2007) and Pseudococcidae (mealybugs) such as Maconellicoccus hirsutus Green, 1908, and Planococcus sp., pests of Ficus carica, and on Nipaecoccus vastator (Maskell, 1895), a pest of Citrus spp. (Martin 1972).

Material examined. Asir: Al Majardah, Wadi Al Talalie, $19^{\circ} 05.19^{\prime} \mathrm{N}, 41^{\circ} 47.78^{\prime} \mathrm{E}$,

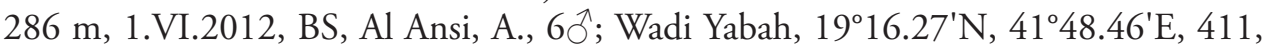
15.IV.2016, SN, Al Ansi, A., 201\%; Thee Ain, $19^{\circ} 55.78^{\prime} \mathrm{N}, 41^{\circ} 26.60^{\prime} \mathrm{E}, 741 \mathrm{~m}$, 5.V.2015, HP, Soliman, A., $10^{\top}$; Baha: Wadi Ghanuna, $19^{\circ} 24.67^{\prime} \mathrm{N}, 41^{\circ} 36.39^{\prime} \mathrm{E}$, 348 m, 11.V.2011, SN, Fadl et al., $20^{\top}$; Jizan: Farasan Island, $16^{\circ} 41.46^{\prime} \mathrm{N}, 42^{\circ} 07.22^{\prime} \mathrm{E}$, 9.II.2012, SN, Deeming, J., $10^{\top}$; Najran: Hubuna Lahumah, $17^{\circ} 50.47^{\prime} \mathrm{N}, 44^{\circ} 16.82^{\prime} \mathrm{E}$, 1212 m, 14.I.2013, BS, Al Ansi et al., 1\%; Riyadh: Dirab, Education Farm KSU, $24^{\circ} 40^{\prime} \mathrm{N}, 46^{\circ} 35^{\prime} \mathrm{E}, 24 . \mathrm{IX} .2011$, LT, Fadl, H., $10^{\top}$; Ad Diriyah, $24^{\circ} 40^{\prime} \mathrm{N}, 46^{\circ} 35^{\prime} \mathrm{E}$, 10.XI, SN, Farhan, 10'; Rawdhet Khoraim, $25^{\circ} 22.98^{\prime} \mathrm{N}, 47^{\circ} 16.71^{\prime} \mathrm{E}, 559 \mathrm{~m}$, II-VIII.2012, 13 3 , 1 , , 1ex, using BS, MT, SN, and LT, on on C. procera, Rhazya steica and L. shawi; Al Ammariyah, 17.X.2013, SN, Sonbati, S., 19; Riyadh, 18.IV.1978, SN, Talhouk et al., ANMA, 3exs; 28.X.1977, SN, ANMA, 6exs; 15.IX.1977, SN, ANMA,

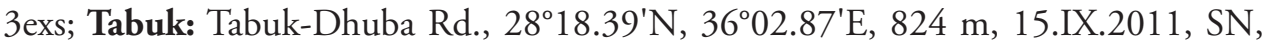
Al Ansi, A., 20'; Qaseem: Buraydah, 26 $12.954^{\circ} \mathrm{N}, 44^{\circ} 02.48^{\prime} \mathrm{E}, 633 \mathrm{~m}, 17 . \mathrm{IX} .2011$, $\mathrm{Al}$ Ansi, A., 20ิ.

Local distribution. It is widely distributed in Saudi Arabia and was collected from Asir, Baha, Jizan, Najran, Riyadh, Tabuk, and Qaseem provinces. Before this study, it was reported by Fürsch (1979) from Riyadh, by Abu-Thuraya (1982) from Riyadh and Madinah, by Talhouk (1982) from Riyadh, and by Abdel-Dayem et al. (2017) from Riyadh; it was listed by Martin (1972).

World distribution. Asia: SA and YE; North Africa: LB; AFR (Kovár 2007); AE (Raimundo et al. 2007), IN (Biranvand et al. 2017).

\section{Scymnini Mulsant, 1846 \\ Clitostethus Weise, 1885}

\section{Clitostethus arcuatus (Rossi, 1794)*}

Figure 4

Coccinella arcuata Rossi, 1794: 88.

Remark. This species is reported as a predator on the whitefly Siphoninus phillyreae (Haliday, 1835) (Hemiptera: Aleyrodidae) in Iran (Tavadjoh et al. 2010).

Diagnosis. Body is dark brown to black, covered with rather long, whitish hairs; head is black; pronotum is whitish, at its middle with several black spots; elytra black or brown, with two arched concentric horseshoe-shaped lines, crossing the suture with their posterior part.

Materials examined. Asir: Ahd Rifidh, $18^{\circ} 06.33^{\prime} \mathrm{N}, 42^{\circ} 53.82^{\prime} \mathrm{E}, 16 . \mathrm{I} .2013$, BS, Al Ansi, A., 1ex.

Local distribution. This is a newly recorded genus from Saudi Arabia; it was collected from Asir province. 


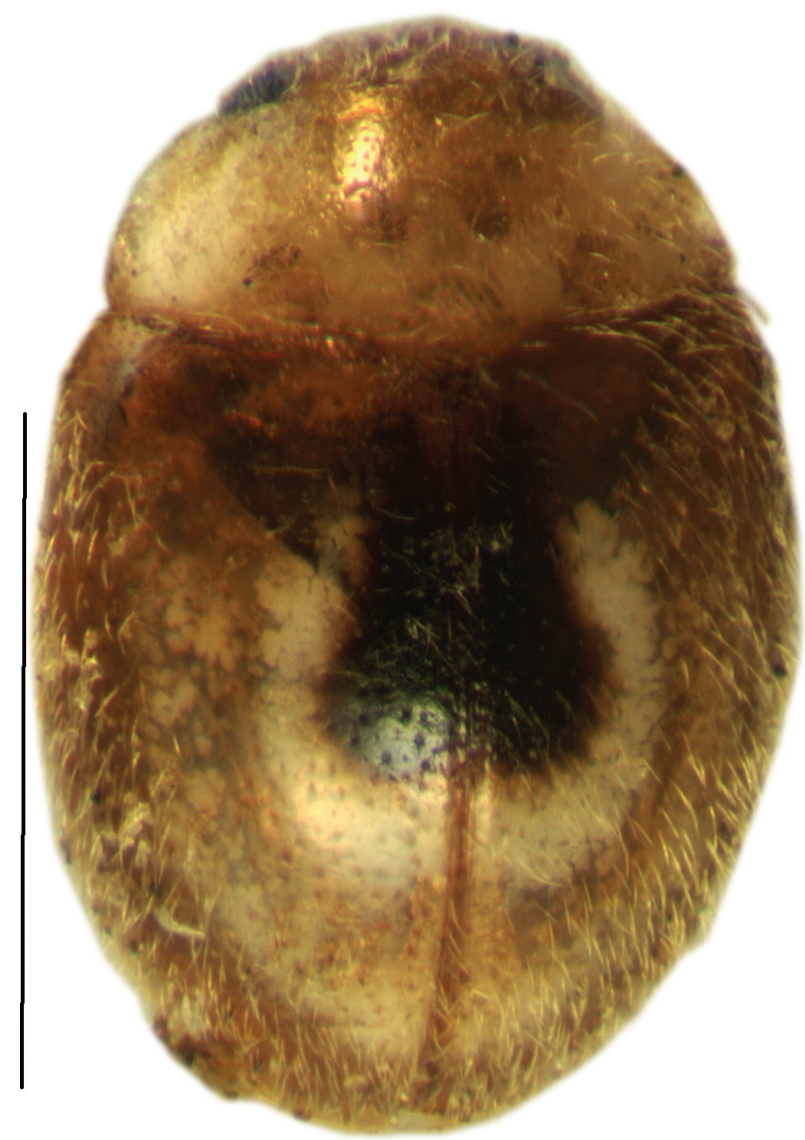

Figure 4. Dorsal view of Clitostethus arcuatus. Scale bar: $1 \mathrm{~mm}$.

World distribution. Asia: IN, IQ, SY, and TR; Europe: AL, AR, AU, AZ, BH, CR, CZ, FR, GB, GE, GG, GR, HU, IT, NL, RO, SK, ST, SZ, UK, and YU; North Africa: CI, and MR; AFR and NAR (Kovár 2007); new record for SA.

\section{Nephus Mulsant, 1846}

Nephus (Bipunctatus) conjunctus (Wollaston, 1870)

Scymnus conjunctus Wollaston, 1870: 248.

Remark. Despite extensive visits throughout the kingdom, this species was not collected during this study period. This species is a predator of aphids (Raimundo et al. 2007).

Local distribution. Asir (Fürsch 1979).

World distribution. E: IT, PT, and SP; N: AG, CI, EG, MO, and TU; A: PAL, SA, and TR; AFR (Kovár 2007); AE (Raimundo et al. 2007). 
Nephus (Bipunctatus) nigricans (Weise, 1879)*

Figure 5

Scymnus (Nephus) bipunctatus nigricans Weise, 1879: 154.

Diagnosis. Body is oval, shining, convex; antennae are 9-segmented; pubescent body, overall black species; each elytron has one large reddish spot in the posterior half.

Materials examined. Asir: Al Majardah Wadi Baqrah Amer Dam, 1847.57'N, $42^{\circ} 01.12^{\prime} \mathrm{E}, 433 \mathrm{~m}, 31$. V.2012, BS, Al Ansi, A., 10'; Baha: Bani Farwah, $20^{\circ} 02.13^{\prime} \mathrm{N}$, 41 ${ }^{\circ} 29.37^{\prime} \mathrm{E}, 2065 \mathrm{~m}, 25 . \mathrm{IV} .2013$, BS, Al Ansi et al., 1\%; Baidah Dam, $20^{\circ} 11.88^{\prime} \mathrm{N}$, 412․06'E, $1880 \mathrm{~m}, 24 . \mathrm{IV} .2013$, BS, Al Ansi et al., $10^{\Uparrow} 1$ 우.

Local distribution. Members of this species were collected from Asir and Baha provinces.

World distribution. Asia: TR and YE (Raimundo and van Harten 2000), new country record for Saudi Arabia; Europe: AL, CR, FR, GR, IT, and TR (Kovár 2007), HU, SK (Haviar and Merkl 2004).

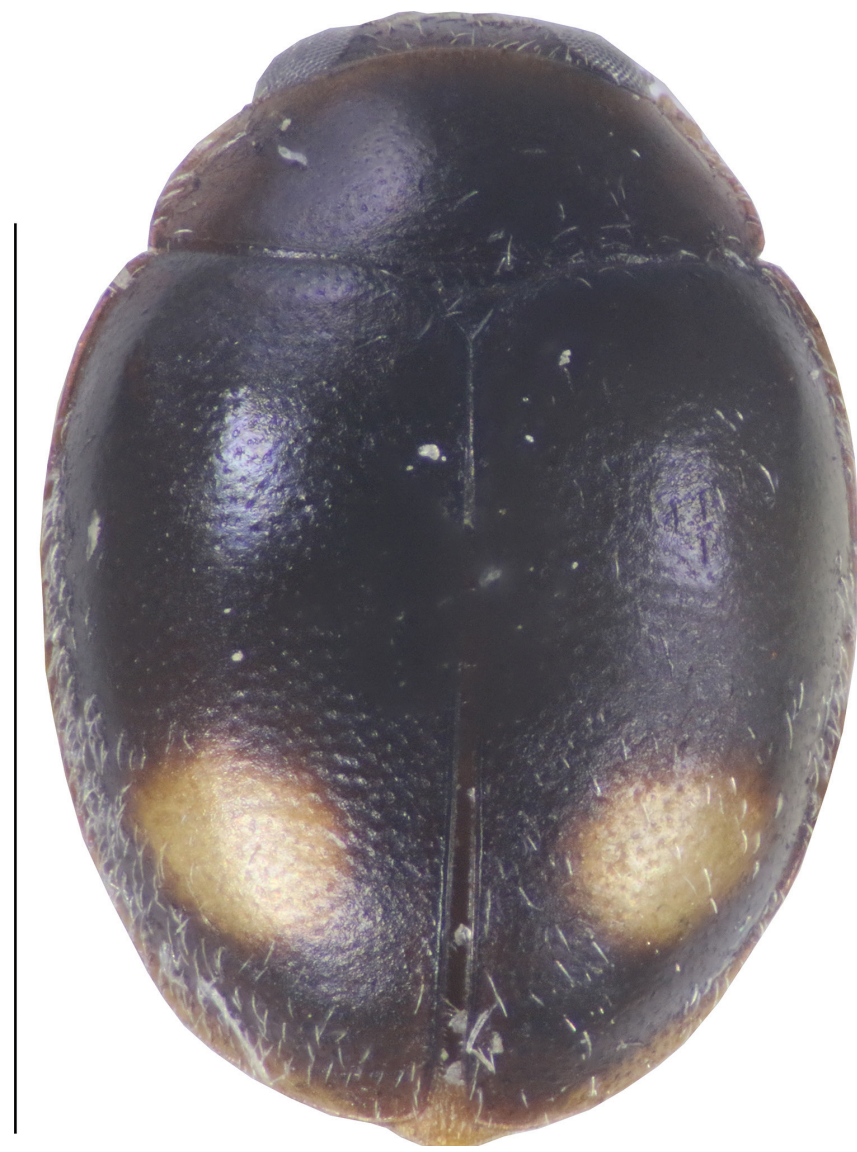

Figure 5. Dorsal view of Nephus nigricans. Scale bar: $1 \mathrm{~mm}$. 


\section{Nephus (Bipunctatus) ornatulus Korschefsky, 1931*}

Figure 6

Nephus (Bipunctatus) ornatulus Korscefsky, 1931: 152.

Diagnosis. Body is elongate oval, covered with whitish pubescence; elytra are generally black, each with two yellowish spots: the anterior one is small or inconspicuous, sometimes invisible, located on the disc, and the posterior one is subapical, large, circular, or transverse.

Materials examined. Asir: Wadi bin Hashbal, $18^{\circ} 35.44^{\prime} \mathrm{N}, 42^{\circ} 39.01^{\prime} \mathrm{E}, 1892 \mathrm{~m}$, 26.IV.2011, SN, Al Ansi et al., 19; Al Magardah Wadi Yabah, 19²16.27'N, 41ํ48.46'E, 411, 2.VI.2012, BS, Al Ansi, A., 10 .

Local distribution. Members of this species were collected from Asir; it was not previously known in Saudi Arabia.

World distribution. Asia: YE, new country record for Saudi Arabia; Africa: Mauritania, Sierra Leone, Cameroon, and Senegal (Raimundo et al. 2006).

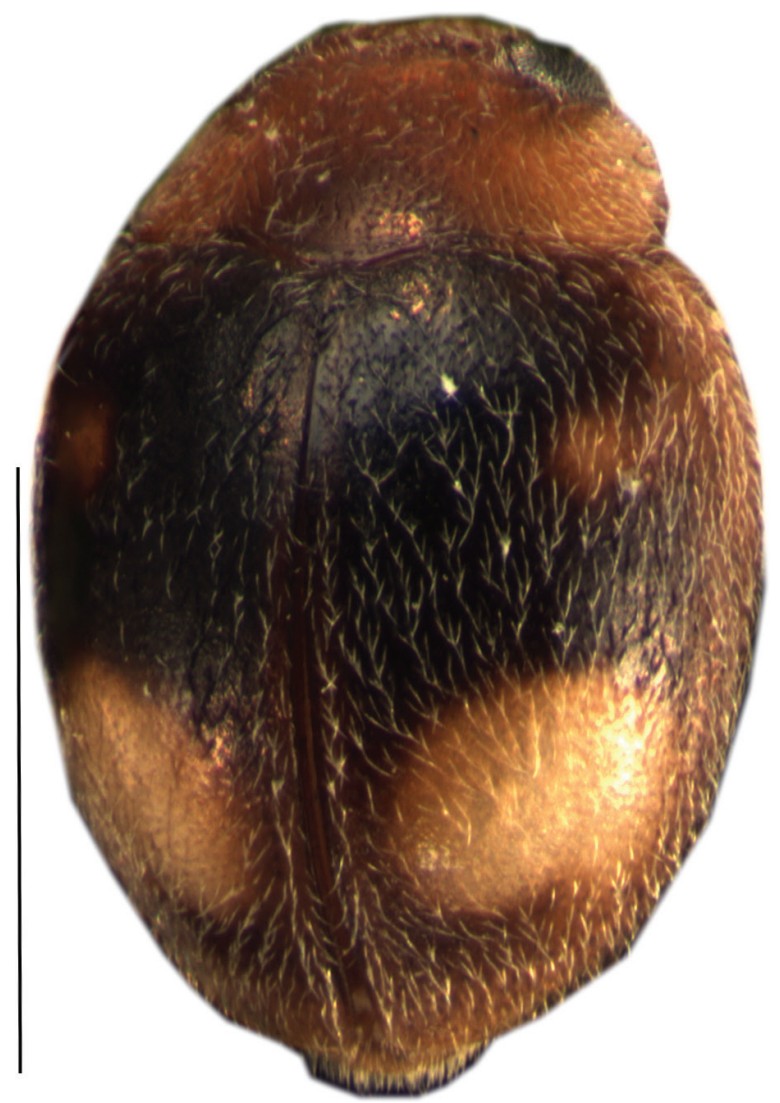

Figure 6. Dorsal view of Nephus ornatulus. Scale bar: $1 \mathrm{~mm}$. 
Nephus (Bipunctatus) wittmeri Fürsch, 1979

Nephus wittmeri Fürsch, 1979: 245.

Remark. This is a rare species reported only from the KSA and in a few Afrotropical countries.

Materials examined. Asir: Al Magardah Wadi Yabah, $19^{\circ} 16.27^{\prime} \mathrm{N}, 41^{\circ} 48.46^{\prime} \mathrm{E}$, 411, 2.VI.2012, BS, Al Ansi, A., 10; Baha: Thee Ain, $19^{\circ} 55.78^{\prime} \mathrm{N}, 41^{\circ} 26.60^{\prime} \mathrm{E}$, 741 m, 11. V.2011, SN, Fadl et al., 1ex; Riyadh: Nursery, 29.IX.2007, SN, Al Dhafer et al., 1ex; Rawdhet Khoraim, $25^{\circ} 22.98^{\prime} \mathrm{N}, 47^{\circ} 16.71^{\prime} \mathrm{E}, 559$ m, 18.II.2012, SN, 4exs, on R. steica.

Local distribution. It was reported from Riyadh by Fürsch (1979); in the present study, it was collected from Asir and Baha. It was also reported from Riyadh by AbdelDayem et al. (2017).

World distribution. A: SA; AFR (Kovár 2007).

Nephus (Geminosipho) arcuatus Kapur, 1959

Nephus (Geminosipho) arcuatus Kapur, 1959: 287.

Remark. This species is found in regions of the Middle East and Africa with an Afrotropical distribution and found in natural habitats. It was generally collected in the months of May and June.

Materials examined. Asir: Al Magardah, Wadi Yabah, $19^{\circ} 16.27^{\prime} \mathrm{N}, 41^{\circ} 48.46^{\prime} \mathrm{E}$, 411, 2.VI.2012, BS, Al Ansi, A., 2exs; Al Magardah, Wadi Khat, $19^{\circ} 05.37^{\prime} \mathrm{N}$, 41 ${ }^{\circ} 58.37^{\prime} \mathrm{E}, 31$. V.2012, BS, Al Ansi, A., 3exs; Baha: Thee Ain, $19^{\circ} 55.78^{\prime} \mathrm{N}$, 41 26.60'E, 741 m, 3.VI.2012, BS, Al Ansi, A., 1 ${ }^{\top}$ 5exs; Jizan: King Faisal Sport City, $16^{\circ} 48.23^{\prime} \mathrm{N}, 42^{\circ} 59.49^{\prime} \mathrm{E}, 11 \mathrm{~m}, 20 . V .2012$, BS, Al Ansi, A., 1 \%; Hurub, Wadi Hurub, $17^{\circ} 43.12^{\prime} \mathrm{N}, 42^{\circ} 57.88^{\prime} \mathrm{E}, 398 \mathrm{~m}, 24 . V .2012$, BS, Al Ansi, A., 10’; Najran: Hubuna, Al Dhaiqah, $17^{\circ} 50.71^{\prime} \mathrm{N}, 4^{\circ} 15.83^{\prime} \mathrm{E}, 1228 \mathrm{~m}, 14$, I.2013, BS, Al Ansi et al., 1ex.

Local distribution. Specimens of this species were collected from Asir, Baha, Jizan and Najran. It was previously reported by Fürsch (1979) from Asir.

World distribution. Asia: IN, SA, and YE; AFR (Kovár 2007); AE (Raimundo et al. 2007).

Nephus (Geminosipho) fenestratus (Sahlberg, 1913)

Scymnus fenestratus Sahlberg, 1913: 83.

Remark. This species was not found during the present study, although it was previously recorded from Riyadh and Jizan provinces. 
Local distribution. Riyadh and Jizan (Fürsch 1979).

World distribution. Asia: SA, SY, and YE (Raimundo and van Harten 2000); AE, CY, IN, PAL, JO, and AE (Raimundo et al. 2007); North Africa: EG; AFR (Kovář 2007).

\section{Nephus (Nephus) crucifer Fleischer, 1900}

Nephus crucifer Fleischer, 1900: 235.

Local distribution. This species was found in Asir, Baha, and Makkah and was also reported by Fürsch (1979) from Asir province.

Materials examined. Asir: Ahd Rifidh, $18^{\circ} 06.33^{\prime} \mathrm{N}, 42^{\circ} 53.82^{\prime} \mathrm{E}, 16.1 .2013$, BS, Al Ansi et al., 3 ${ }^{\top} 3$ \% ; Baha: Al Mikhwah, $1^{\circ} 49.44^{\prime} \mathrm{N}, 41^{\circ} 22.85^{\prime} \mathrm{E}, 430 \mathrm{~m}, 18 . \mathrm{V} .2010$,

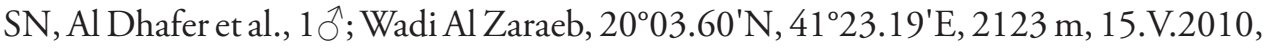

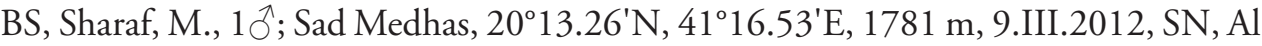

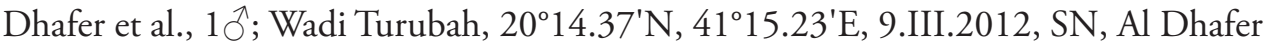
et al., 1; Shabreqah station, $20^{\circ} 08.03^{\prime} \mathrm{N}, 41^{\circ} 23.60^{\prime} \mathrm{E}, 2267 \mathrm{~m}, 24 . \mathrm{IV} .2013$, BS, Al

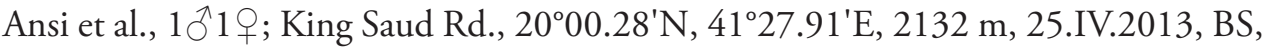
Al Ansi et al., $2{ }^{\top} 2{ }^{\circ}$; Shuaib Wadi Turubah, $20^{\circ} 14.37^{\prime} \mathrm{N}, 41^{\circ} 15.23^{\prime} \mathrm{E}, 24 . \mathrm{IV} .2013$, BS,

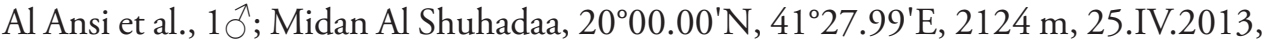
BS, Al Ansi et al., 19; Rahwan $10 \mathrm{~km}$ outside of Baha, $20^{\circ} 04.03^{\prime} \mathrm{N}, 41^{\circ} 27.01^{\prime} \mathrm{E}$,

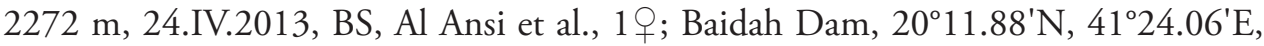
1880 m, 24.IV.2013, BS, Al Ansi et al., 1ð`; Al Lishtah Village 20 km outside of Baha, 26.IV.2013, BS, Al Ansi et al., 1ठ̄; Makkah: Baha-Taif Rd. Bani Malik Wadi Rakhmat, $20^{\circ} 42.15^{\prime} \mathrm{N}, 40^{\circ} 59.46^{\prime} \mathrm{E}, 1616 \mathrm{~m}, 20^{\circ} 42.15^{\prime} \mathrm{N}, 40^{\circ} 59.46^{\prime} \mathrm{E}, 1616 \mathrm{~m}, 4 . \mathrm{VI} .2012$, BS, Al Ansi, A., 1 .

World distribution. Asia: IN, SA, TD, UZ, and KZ; AFR (Kovár 2007).

\section{Nephus (Sidis) hiekei (Fürsch, 1965)}

Scymnus hiekei Fürsch, 1965: 201.

Remark. This species is known as a predator of the mealybug Planococcus citri (Risso, 1813 (Fürsch 1970, Abu-Thuraya 1982, Raimundo and van Harten 2000).

Materials examined. Riyadh: Ad Diriyah, 244' $\mathrm{N}, 46^{\circ} 35^{\prime} \mathrm{E}, 17 . \mathrm{XI} .2011$, SN, Al Omar, A., 1ex; 15.IX.2011, SN, Al Omar, A., 1ex; Al Ammariah, 24²9'N, 46 26'E, 24.XI.2009, SN, Sharaf, M., 1ex.

Local distribution. It is restricted to Riyadh province and previously reported from Riyadh by Fürsch (1979) and Abu-Thuraya (1982) and listed by Martin (1972) and Talhouk (1982). In this study, it was collected from Riyadh.

World distribution. Asia: AE, IN, IQ, PAL, LE, SA, and TR; Europe: IT, GR, and SP; North Africa: AG, EG, MO, and TU (Kovář 2007). 


\section{Nephus (Sidis) levaillanti (Mulsant, 1850)}

Scymnus levaillanti Mulsant, 1850: 964.

Remark. The specimens were collected by PT on the branches of Acacia gerrardii and under the shrub $Z$. nummularia. Some specimens were also collected under the canopies of Acacia ehrenbergiana during April and July. This species is a predator on Aphis gossypii Glover, 1877 (Fürsch 1970) and A. orientalis (Abu-Thuraya 1982).

Materials examined. Riyadh: Rawdhet Khoraim, $25^{\circ} 22.98^{\prime} \mathrm{N}, 47^{\circ} 16.71^{\prime} \mathrm{E}$,

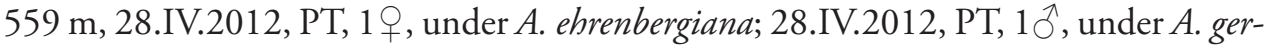
rardii; 1.VII.2012, BS, 1 , on $A$. gerrardii; 14.IV.2012, BS, 1ex, on $Z$. nummularia.

Local distribution. Specimens were collected from Riyadh (Abdel-Dayem et al. 2017) and also previously was reported from Riyadh and Eastern provinces by AbuThuraya (1982) and listed by Martin (1972).

World distribution. Asia: SA (Raimundo and van Harten 2000); AF, FE, IN, PAL, JA, JO, LE, PA, SHA, and TAI; Europe: GR and IT; North Africa: EG; AFR and ORR (Kovář 2007).

\section{Scymnus Kugelann, 1794}

Scymnus (Pullus) agrumi Fürsch, 1970

Scymnus (Pullus) agrumi Fürsch, 1970: 109.

Remark. This is a very rare species that is endemic to Saudi Arabia and found to be a predator of the mealybug P. citri (Fürsch 1970, Martin 1972; Abu-Thuraya 1982).

Material examined. Eastern region: Qatif, $26^{\circ} 45^{\prime} \mathrm{N}, 49^{\circ} 58^{\prime} \mathrm{E}, 1969$, Fürsch, ANMA, 1ex, paratype.

Local distribution. It was described from the Eastern province of KSA by Fürsch (1970), reported by Abu-Thuraya (1982) also from Eastern Province, and listed by Martin (1972).

World distribution. Asia: Endemic to SA (Fürsch 1970; Kovár 2007).

\section{Scymnus (Pullus) arabicus Fürsch, 1989}

Scymnus arabicus Fürsch, 1989: 116.

Remark. This is also a rare species that has not been collected since its description by Fürsch (1989). It is endemic to Saudi Arabia.

Local distribution. It was found in Asir province (Fürsch 1989).

World distribution. Asia: Endemic to SA (Fürsch 1989; Kovár 2007). 
Scymnus (Pullus) auritus Thunberg, 1795

Scymnus (Pullus) auritus Thunberg, 1795: 105.

Remark. Presence of this species was not confirmed, except in the catalogue of Kovár (2007) with insufficient details regarding its distribution in Saudi Arabia. However, it is well distributed around the world.

World distribution. Asia: ANH, CY, ES, FE, FUJ, GUA, IN, SA, SCH, SY, TR, and WS; Europe: AL, AU, BH, BU, BY, CR, CT, CZ, DE, EN, FI, FR, GB, GE, GG, GR, HU, LA, LT, LU, MC, MD, NR, NT, PL, PT, RO, SK, SL, SP, ST, SV, SZ, TR, UK, and YU (Kovář 2007).

Scymnus (Pullus) ebneri Weise, 1926

Scymnus ebneri Weise, 1926: 228.

Remark. This is also a rare species and known as a predator of aphids (Abu-Thuraya 1982; Talhouk 1982).

Material examined. Eastern province: Qatif, $26^{\circ} 45^{\prime} \mathrm{N}, 49^{\circ} 58^{\prime} \mathrm{E}, 5 . \mathrm{VIII} .1970$, det. R. D. Pope, 1971, ANMA, 1ex, on alfalfa.

Local distribution. It was reported from Riyadh by Abu-Thuraya (1982) and listed by Martin (1972) and Talhouk (1982).

World distribution. Asia: AE, IN, and SA (Abu-Thuraya 1982). North Africa: EG; AFR (Kováŕ 2007).

Scymnus (Pullus) latemaculatus Motschulsky, 1858

Scymnus (Pullus) latemaculatus Motschulsky, 1858: 121.

Remark. Specimens of this species could not be examined due to the unavailability of material.

Local distribution. This species was reported from Riyadh by Fürsch (1979).

World distribution. Asia: AF, FUJ, GUA, GUX, IN, PA, TAI, ZHE, and SA; North Africa: LB; ORR (Kovár 2007); AE (Raimundo et al. 2007).

Scymnus (Pullus) luxorensis Fürsch, 1989

Scymnus (Pullus) luxorensis Fürsch, 1989: 116.

Remark. This is a rare species with limited geographical distribution. It was collected from L. shawii using a beating sheet in October; it is also not common in its type locality range. 


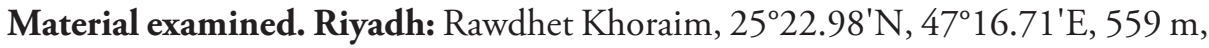
30.X.2011, BS, Al Ansi, A., 10, on L. shawii.

Local distribution. It was described from Riyadh by Fürsch (1989) and also collected again from Riyadh by Abdel-Dayem et al. (2017).

World distribution. Asia: It was described from SA (Fürsch 1989) and also known in North Africa: EG (Kovár 2007).

\section{Scymnus (Pullus) subvillosus (Goeze, 1777)}

\section{Coccinella subvillosus Goeze, 1777: 247.}

Remark. This is a very common species found throughout the year in SA and also widely distributed throughout the most world countries. This species was primarily collected from the habitats of A. ehrenbergiana, A. gerrardii, C. procera, L. shawii, $R$. stricta, and $Z$. nummularia.

Material examined. Asir: Al Majardah Wadi AlTalalie, $1^{\circ} 05.19^{\prime} \mathrm{N}, 41^{\circ} 47.78^{\prime} \mathrm{E}, 286$ m, 1.VI.2012, BS, Al Ansi, A., 1ex; Al Majardah Wadi Khat, $19^{\circ} 05.37^{\prime} \mathrm{N}, 41^{\circ} 58.37^{\prime} \mathrm{E}$, 13.III.2012, BS, Abdel-Dayem et al., 3exs; 31.V.2012, BS, Al Ansi, A., 4exs; Al Majardah Wadi Qanunah, $19^{\circ} 24.67^{\prime} \mathrm{N}, 41^{\circ} 36.39^{\prime} \mathrm{E}, 348 \mathrm{~m}, 11 . \mathrm{III} .2012$, SN, Al Dhafer et al., 1ex; 11.III.2012, LT, Al Dhafer et al., 1ex; Al Majardah, Wadi Yabah, 19 ${ }^{\circ} 16.27^{\prime} \mathrm{N}$,

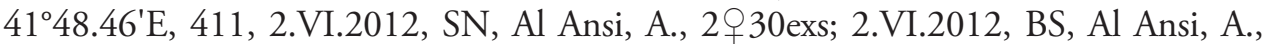
$1{ }^{\Uparrow} 4$ + $37 \mathrm{exs}$; 12.III.2012, SN, Al Dhafer et al., 1ex; Ahd Rifidh, $18^{\circ} 06.33^{\prime} \mathrm{N}, 42^{\circ} 53.82^{\prime} \mathrm{E}$, 16.I.2013, SN, Al Ansi et al., 2exs; Baha: Thee Ain, $19^{\circ} 55.78^{\prime} \mathrm{N}, 41^{\circ} 26.60^{\prime} \mathrm{E}, 741 \mathrm{~m}$, 11.V.2011, SN, Fadl et al., 1ex; 10.III.2012, SN, Al Dhafer et al., 10 ,2exs; 3.VI.2012, BS, Al Ansi, A., 1ex; Jizan: Al Aydabi Jaorat Aiban, $17^{\circ} 25.53^{\prime} \mathrm{N}, 43^{\circ} 03.50^{\prime} \mathrm{E}, 343 \mathrm{~m}$, $17^{\circ} 25.53^{\prime} \mathrm{N}, 43^{\circ} 03.50^{\prime} \mathrm{E}, 343$ m, 23.V.2012, BS, Al Ansi, A., 2exs; Najran: Rijla Wadi Najran, $17^{\circ} 31.56^{\prime} \mathrm{N}, 4^{\circ} 13.65^{\prime} \mathrm{E}, 1257$ m, 15.I.2013, BS, Al Ansi et al., 15exs; Wadi Shuaib Barran, $17^{\circ} 28.94^{\prime} \mathrm{N}, 4^{\circ} 05.52^{\prime} \mathrm{E}, 1325 \mathrm{~m}$, 16.I.2013, BS, Al Ansi et al., 7exs; Hubuna Al Dhaiqah, $17^{\circ} 50.71^{\prime} \mathrm{N}, 4^{\circ} 15.83^{\prime} \mathrm{E}, 1228 \mathrm{~m}$, 14.I.2013, BS, Al Ansi et al., 11 exs; Hubuna Lahumah, $17^{\circ} 50.47^{\prime} \mathrm{N}, 4^{\circ} 16.82^{\prime} \mathrm{E}, 1212 \mathrm{~m}$, 14.I.2013, BS, Al Ansi et al., 75exs; Riyadh: Al Muzahimiyah Al Khararah, $4^{\circ} 24.21^{\prime} \mathrm{N}, 46^{\circ} 14.40^{\prime} \mathrm{E}, 30 . I I I .2011$, SN, Al Dryhim et al., 9exs; 17.IV.2012, LT, Al Dhafer et al., 3exs; Rawdhet Khoraim, $25^{\circ} 22.98^{\prime} \mathrm{N}, 47^{\circ} 16.71^{\prime} \mathrm{E}, 559 \mathrm{~m}, 182$ exs collected by $\mathrm{SN}$ and B on branches of and by PT under canopy of $A$. ehrenbergiana, A. gerrardii, C. procera, L. shawii, R. stricta, $Z$. nummularia; ; also by LT; XII.2011; I, III-VII, IX, XI.2012; I-IX.2013; 11.IV.2011, SN, Al Dryhim et al., 1ex; 11.IV.2011, SN, Al Dryhim et al., 1ex; Hutet Bani Tamim Al Hareeq Rd., 2332.03'N, 46²7.33'E, 584 m, 8.V.2012, LT, Al Ansi, A., 1ex; Hutet Bani Tamim Ibex Reserve National Park, $23^{\circ} 28.10^{\prime} \mathrm{N}, 46^{\circ} 36.35^{\prime} \mathrm{E}, 28 . X I I .2007$, SN, Al Dryhim et al., 1ex; 19.IV.2008, SN, 4exs; 19.V.2007, SN, 2exs; 21.III.2008, ST, 1ex; 21.III.2008, SN, 1ex; 4.IV.2008, SN, 2exs; Wadi Ad Dawasir, $20^{\circ} 25.83^{\prime} \mathrm{N}, 4^{\circ} 56.53^{\prime} \mathrm{E}$, 641 m, 17.I.2013, BS, Al Ansi et al., 1ex; Wadi Namar, 243․ ${ }^{\circ} .18^{\prime} \mathrm{N}, 46^{\circ} 34.60^{\prime} \mathrm{E}$, 29.II.2012, HP, Al Ansi, A., 1ex; Dirab, $23^{\circ} 30^{\prime} \mathrm{N}, 46^{\circ} 51^{\prime} \mathrm{E}, 26 . I V .2010$, SN, Rashid, O., 1ex; 14.III.2010, ST, Al Dhafer et al., 1ex; 25.IV.2010, ST, Al Dhafer et al.,1ex; 
28.III.2010, ST, Al Dhafer et al.,2exs; 20.XII.2009, ST, Al Dhafer et al.,1ex; Riyadh Nursery, 16.V.2007, SN, Al Dhafer et al., 1ex; 29.IX.2007, SN, Al Dhafer et al.,1ex; Al Waseel, $24^{\circ} 48.40^{\prime} \mathrm{N}, 46^{\circ} 30.42^{\prime} \mathrm{E}, 20 . X$.2011, SN, Al Rashide, H., 1ex; Ad Diriyah Education Farm, $24^{\circ} 40^{\prime} \mathrm{N}, 46^{\circ} 35^{\prime} \mathrm{E}, 21 . X .2009$, SN, Salah, A., 1ex; Al Ahsa, $22^{\circ} 17.53^{\prime} \mathrm{N}$, 5040.46'E, 28.XII.1982, Al Shuaaei, M., ANMA, 1ex.

Local distribution. It was collected from different localities of Asir, Baha, Eastern Province, Jizan, Najran, and Riyadh provinces and previously reported by Fürsch (1979) and Abdel-Dayem et al. (2017).

World distribution. Asia: AE, AF, CY, IN, IQ, PAL, JO, KI, KU, KZ, LE, PA, QA, SA, SI, SY, TD, TR, UZ, and YE; Europe: AB, AL, AN, AR, AU, AZ, BH, BU, CR, CZ, FR, GE, GG, GR, HU, IT, MC, PT, RO, SK, SL, SP, ST, SZ, TR, UK, and YU; North Africa: AE, AG, CI, EG, LB, MO, MR, and TU; AFR (Kovár 2007).

\section{Scymnus (Pullus) syriacus (Marsuel, 1868)}

Nephus syriacus Marsuel, 1868: 216.

Remark. This is a widely distributed species throughout the year in SA and was collected from the habitats of A. ehrenbergiana, A. gerrardii, C. procera, L. shawii, R. stricta, and Z. nummularia. It is a predator on Aphis fabae Scopoli, 1840 (Talhouk 1982; Fürsch 1989) and whiteflies and aphids (Martin 1972).

Material examined. Riyadh: Hutet Bani Tamim Ibex Reserve National Park, $23^{\circ} 28.10^{\prime} \mathrm{N}, 46^{\circ} 36.35^{\prime} \mathrm{E}, 4 . I \mathrm{IV} .2008$, SN, Al Dryhim et al., 2`30exs; 21.III.2008, SN, 2ㅇexs; 28.VII.2007, SN, 1ex; 19.V.2007, SN, 2exs; 19.IV.2008, SN, 1ex; 7.V.2012, LT, Al Ansi, A., 1ex; 28.V.2010, SN, Al Dhafer et al., 2exs; Hutet Bani Tamim-Al Hareeq Rd., 2332.03'N, 46⒋33'E, 584 m, 8.V.2012, LT, Al Ansi,A., 1 우 3exs; Al Hareeq-Ar Rayn Rd., $23^{\circ} 33.88^{\prime} \mathrm{N}, 46^{\circ} 22.53^{\prime} \mathrm{E}, 737$ m, 8.V.2012, LT, Al Ansi, A., 10'; Hutet Bani Tamim, 23⒉26'N, 46⒋13'E, 19.IV.2008, SN, Al Dhafer, H., 7exs; 7.V.2012, HP,

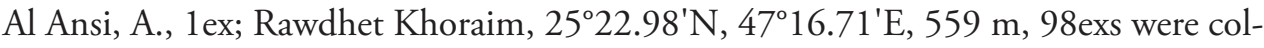
lected by $\mathrm{SN}$ and $\mathrm{B}$ on branches of A. ehrenbergiana, A. gerrardii, C. procera, L. shawii, $R$. stricta and $Z$. nummularia; and HP under canopy of the previous plants; also by LT; through X, XII.2011; II-VI, X-XII.2012; III, IV, VI, VII.2013; 11.IV.2011, SN, Al Dryhim et al., 1क; 11.IV.2011, SN, Al Dryhim et al., 1ex; 16.X.2011, LT, 1ex; 26.V.2012, BS, $1 \delta^{\Uparrow} 1 \mathrm{ex} ; 26 . V .2012$, LT, $1 \overbrace{}^{\Uparrow} 1 \mathrm{ex} ; 18 . I I .2012$, SN, 1ex; 26.V.2012, BS, 1ठ1ex; 15.V.2012, SN, 1ex; 14.V.2012, BS, 1ex; 14.V.2012, SU, 2exs; 14.V.2012, BS, 1ex; Wadi Namar, $24^{\circ} 32.18^{\prime} \mathrm{N}, 46^{\circ} 34.60^{\prime} \mathrm{E}$, 29.II.2012, BS, Al Ansi et al., 1ex; Al Kharj, 20 24'N, 46 29'E, 18.XI.2009, SN, Al Dryhim et al., 2exs; Wadi Ad Dawasir,

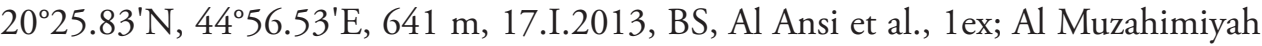
Al Khararah, $24^{\circ} 24.21^{\prime} \mathrm{N}, 46^{\circ} 14.40^{\prime} \mathrm{E}, 30 . \mathrm{III} .2011$, SN, Al Dryhim et al., $10^{\Uparrow} 1$ \% 4 exs; Najran: Hubuna Al Dhaiqah, $17^{\circ} 50.71^{\prime} \mathrm{N}, 4^{\circ} 15.83^{\prime} \mathrm{E}, 1228$ m, 14.I.2013, BS, Al Ansi et al., 2exs; Rijla Wadi Najran, $17^{\circ} 31.56^{\prime} \mathrm{N}, 4^{\circ} 13.65^{\prime} \mathrm{E}, 1257 \mathrm{~m}, 15 . \mathrm{I} .2013$, BS, 2exs; Hubuna Lahumah, $17^{\circ} 50.47^{\prime} \mathrm{N}, 4^{\circ} 16.82^{\prime} \mathrm{E}, 1212 \mathrm{~m}$, 14.I.2013, BS, 4exs. 
Local distribution. Specimens of this species were collected from Eastern, Najran, and Riyadh provinces and have been previously reported from Eastern Province by Talhouk (1982) and Fürsch (1989), from Eastern province and Baha by El-Hawagry et al. (2013), and from Riyadh by Abdel-Dayem et al. (2017); it was listed by Martin (1972).

World distribution. Asia: SA (Fürsch 1989); Asia: CY, IN, IQ, PAL, JO, LE, SI, and SY; North Africa: EG; (Kovár 2007).

\section{Scymnus (Pullus) yemenensis (Kapur, 1959)}

Pullus yemenensis Kapur, 1959: 281.

Remark. This species is distributed only in the Arabian Peninsula and is generally found in the natural habitats of $A$. ehrenbergiana and $C$. procera in the vicinity of hemipterous insects.

Material examined. Asir: Abha, Habalah, $18^{\circ} 02.05^{\prime} \mathrm{N}, 42^{\circ} 51.49^{\prime} \mathrm{E}, 25 . \mathrm{IV} .201$ SN, Al Ansi et al., $5{ }^{\wedge} 191$ ex; Ahd Rifidh, $18^{\circ} 06.33^{\prime} \mathrm{N}, 42^{\circ} 53.82^{\prime} \mathrm{E}, 16 . \mathrm{I} .2013$, BS,

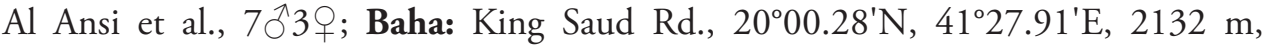
25.IV.2013, BS, Al Ansi et al., 4§1 1 ; Al Leishtah Village $20 \mathrm{~km}$ outside of Baha, $20^{\circ} 12.16^{\prime} \mathrm{N}, 41^{\circ} 22.21^{\prime} \mathrm{E}, 2231 \mathrm{~m}, 26 . I V .2013$, BS, $10^{\top}$; Baidah Dam, $20^{\circ} 11.88^{\prime} \mathrm{N}$, $41^{\circ} 24.06^{\prime} \mathrm{E}, 1880 \mathrm{~m}, 24 . \mathrm{IV} .2013$, BS, 20 1 \% ; Midan Al Shuhadaa, $20^{\circ} 00.00^{\prime} \mathrm{N}$, $41^{\circ} 27.99^{\prime} \mathrm{E}, 2124 \mathrm{~m}, 25 . \mathrm{IV} .2013$, BS, $2{ }^{\circ} 5$ 웅 Wadi Turubah, $20^{\circ} 14.37^{\prime} \mathrm{N}, 41^{\circ} 15.23^{\prime} \mathrm{E}$, 26.IV.2013, BS, $2{ }^{\lambda} 2$; ; Rahwan, $10 \mathrm{~km}$ outside of Baha, $20^{\circ} 04.03^{\prime} \mathrm{N}, 41^{\circ} 27.01^{\prime} \mathrm{E}$, $2272 \mathrm{~m}, 24 . \mathrm{IV} .2013$, BS, 1 \% ; Thee Ain, $19^{\circ} 55.78^{\prime} \mathrm{N}, 4^{\circ} 26.60^{\prime} \mathrm{E}, 741 \mathrm{~m}, 25 . \mathrm{IV} .2013$, BS, 1; Shabreqah Station, $20^{\circ} 08.03^{\prime} \mathrm{N}, 4^{\circ} 23.60^{\prime} \mathrm{E}, 2267 \mathrm{~m}$, 24.IV.2013, BS, 4우 Wadi Turubah, $20^{\circ} 14.37^{\prime} \mathrm{N}, 41^{\circ} 15.23^{\prime} \mathrm{E}, 14 . \mathrm{V} .2011$, SN, Al Dhafer, H., Fadl, H.,

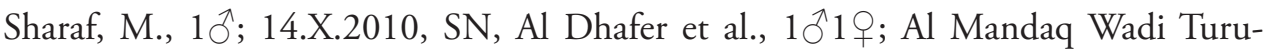
bah, $20^{\circ} 14.37^{\prime} \mathrm{N}, 41^{\circ} 15.23^{\prime} \mathrm{E}, 3 . \mathrm{VI} .2012$, BS, Al Ansi, A., 10'; Najran: Al Mofejah, $17^{\circ} 27.25^{\prime} \mathrm{N}, 44^{\circ} 03.48^{\prime} \mathrm{E}, 1263 \mathrm{~m}$, 16.I.2013, BS, Al Ansi et al., 1 1 $^{\circledR}$; Rijla Wadi Najran, $17^{\circ} 31.56^{\prime} \mathrm{N}, 44^{\circ} 13.65^{\prime} \mathrm{E}, 1257 \mathrm{~m}, 15 . \mathrm{I} .2013$, BS, 20 1 ; Hubuna Lahu-

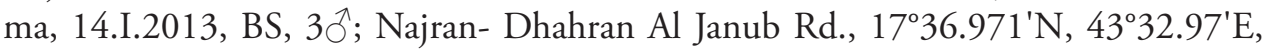
2132 m, 16.I.2013, BS, 1; Riyadh: Hawtet Bani Tameem- Al Hariq Rd. 15 km, 8.V.2012, LT, Al Ansi, A., 10'; Wadi Namar, $24^{\circ} 32.18^{\prime} \mathrm{N}, 46^{\circ} 34.60^{\prime} \mathrm{E}, 29.1 \mathrm{II} .2012$,

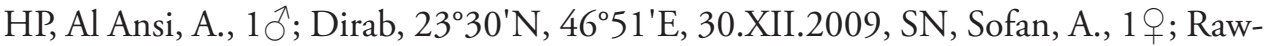
dhet Khoraim, $25^{\circ} 22.98^{\prime} \mathrm{N}, 47^{\circ} 16.71^{\prime} \mathrm{E}, 559 \mathrm{~m}$, 14.IV.2012, BS, $10^{1}$ on A. ehrenbergiana; 28.IV.2012, LT, 19; 14.IV.2012, SN, 10̂; 13.X.2012, LT, 1ex, on C. procera; 13.X.2012, SN, 5exs, on C. procera.

Local distribution. It was collected from Asir, Baha, Najran, and Riyadh and was previously reported by Fürsch (1979) from Asir and Riyadh and from Riyadh by Fürsch (1989) and also by Abdel-Dayem et al. (2017).

World distribution. Asia: OM, SA, and YE (Kovár 2007); AE (Raimundo et al. 2007). 


\title{
Scymnus (Scymnus) interruptus (Goeze, 1777)
}

\author{
Coccinella interrupta Goeze, 1777: 247.
}

Remark. This species is widely distributed in SA and commonly found in natural habitats.

Materials examined. Asir: Muhail Wadi Hali, $18^{\circ} 30.12^{\prime} \mathrm{N}, 42^{\circ} 02.21^{\prime} \mathrm{E}, 440 \mathrm{~m}$, 11.II.2016, SU, Al Ansi, A., 30exs; Maraba Wadi Reem, $17^{\circ} 52.55^{\prime} \mathrm{N}, 42^{\circ} 16.66^{\prime} \mathrm{E}$, 136 m, 9.II.2016, SU, Al Ansi, A., 41exs; Maraba Wadi Ramlan, $17^{\circ} 47.18^{\prime} \mathrm{N}$, $42^{\circ} 22.95^{\prime} \mathrm{E}, 180 \mathrm{~m}, 10 . \mathrm{II} .2016$, SU, Al Ansi, A., 19exs; Maraba Wadi Itwad, $17^{\circ} 48.25^{\prime} \mathrm{N}, 42^{\circ} 21.64^{\prime} \mathrm{E}, 149$ m,8.II.2016, SU, Al Ansi, A., 29exs; Rijal Alma Wadi Sabin, $17^{\circ} 48.25^{\prime} \mathrm{N}, 42^{\circ} 21.64^{\prime} \mathrm{E}, 194 \mathrm{~m}$, 10.II.2016, SU, Al Ansi, A., 12exs; Wadi Maraba, $18^{\circ} 10.29^{\prime} \mathrm{N}, 42^{\circ} 22.19^{\prime} \mathrm{E}, 1150 \mathrm{~m}, 16 . I V .2016$, LT, Abdel-Dayem et al., 1ex; Raghdan, $20^{\circ} 34.25^{\prime} \mathrm{N}, 41^{\circ} 45.11^{\prime} \mathrm{E}, 10 . \mathrm{IV} .2016$, SU, Al Ansi, A., 4exs; Abha, Raydah, $18^{\circ} 12.10^{\prime} \mathrm{N}, 42^{\circ} 24.54^{\prime} \mathrm{E}, 2578 \mathrm{~m}$, 16.IV.2016, SN, Soliman, A., 1ex; Thalooth Al Mandhar Wadi Baqrah, 18 $47.57^{\prime} \mathrm{N}, 42^{\circ} 01.12^{\prime} \mathrm{E}, 433 \mathrm{~m}, 12 . \mathrm{X} .2013$, SN,

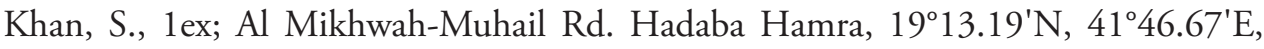
374 m, 15.IV.2016, SN, Al Ansi, A., 1ex; Baha: Al Mikhwah Thee Ain, $19^{\circ} 55.78^{\prime} \mathrm{N}$,

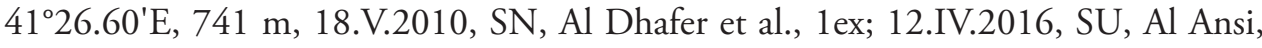
A., 27exs; Wadi Turubah, $20^{\circ} 14.37^{\prime} \mathrm{N}, 41^{\circ} 15.23^{\prime} \mathrm{E}, 10 . \mathrm{V} .2011$, SN, Fadl et al., 1ex; Thee Ain, $19^{\circ} 55.78^{\prime} \mathrm{N}, 41^{\circ} 26.60^{\prime} \mathrm{E}, 741 \mathrm{~m}, 13 . \mathrm{X} .2010$, SN, Al Dhafer et al., 1ex; 7.III.2013, SN, Al Harbi et al., 1ex; 7.III.2013, BS, Al Harbi et al., 1 ex; Jizan: Sabya-

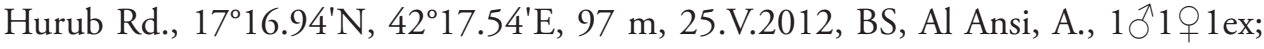
Jizan-Abu Arish Rd., $17^{\circ} 04.25^{\prime} \mathrm{N}, 42^{\circ} 47.05^{\prime} \mathrm{E}, 24 . I I .2015$, SN, Al Harbi, M., 2exs; LT, 3exs; HP, 1ex; Najran: Rijla Wadi Najran, $17^{\circ} 31.56^{\prime} \mathrm{N}, 44^{\circ} 13.65^{\prime} \mathrm{E}, 1257 \mathrm{~m}$, 15.I.2013, BS, Al Ansi et al., 3exs.

Local distribution. It was collected from Asir, Baha, Jizan, and Najran and was previously reported by Fürsch (1979) in the taxonomic key.

World distribution. Asia: IQ, PAL, JO, KZ, MG, TR, SY, and YE; Europe: AB, AL, AR, AU, BE, BU, BY, CR, CZ, FR, GE, GG, GR, HU, IT, UZ, WS, XIN, PT, RO, SK, SP, SZ, TR, and UK; North Africa: AG, EG, LB, MO, MR, and TU (Kovár 2007).

\section{Scymnus (Scymnus) nubilus Mulsant, 1850}

Scymnus nubilus Mulsant, 1850: 972.

Remark. This species is found in both natural habitats and agroecosystems and collected throughout the year in SA. It is a well-known predator of the sugarcane whitefly, Aleurolobus barodensis (Maskell, 1896) (Hodek and Honek 2009) and aphids (Raimundo et al. 2007).

Material examined. Asir: Wadi Abha, $18^{\circ} 22.03^{\prime} \mathrm{N}, 42^{\circ} 50.82^{\prime} \mathrm{E}, 1990 \mathrm{~m}$, 28.IV.2011, SN, Al Ansi et al., 1ex; Wadi Ghanuna, 19²4.67' N, 41 $36.39^{\prime} \mathrm{E}, 348 \mathrm{~m}$, 
11.V.2011, SN, Fadl et al., 1ex; Al Majaridah, Wadi Yabah, $19^{\circ} 16.27^{\prime} \mathrm{N}, 41^{\circ} 48.46^{\prime} \mathrm{E}$, 411, 2.VI.2012, SN, Al Ansi, A, 13 texs.; 2.VI.2012, BS, Al Ansi, A., 4exs; Al Ma-

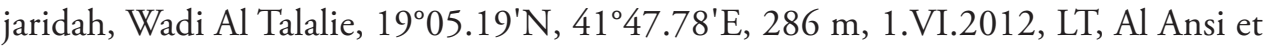
al., 1ex; 1.VI.2012, SN, Al Ansi, A., 2exs; Khamis Mushait, Wadi Bisha, 18²0.01'N, 4242.13'E, 1990 m 27.IV.2011, SN, Al Ansi, A., 3exs; Ahd Rifidh, $18^{\circ} 06.33^{\prime} \mathrm{N}$, 4253.82'E, 16.I.2013, BS, Al Ansi, A., 1ex; 16.I.2013, SN, Al Ansi, A., 4exs; Thalooth Al Mandhar Wadi Baqrah, 1847.57'N, 4201.12'E, 433 m, 4.XI.2013, SN, Al Dhafer et al., 1ex; LT, 1ex; 12.X.2013, HP, El Torkey, A., 1ex; Baha: Qilwah-Adhom, Wadi Al Arg, $20^{\circ} 29.98^{\prime} \mathrm{N}, 40^{\circ} 48.95^{\prime} \mathrm{E}, 429$ m, 9.XI.2012, BS, Fadl, H., 1ex; Thee Ain, $19^{\circ} 55.78^{\prime} \mathrm{N}, 41^{\circ} 26.60^{\prime} \mathrm{E}, 741 \mathrm{~m}, 15 . \mathrm{V} .2011$, SN, Fadl et al., 3exs; 3.VI.2012, SN, Al Ansi, A., 2exs; Ghabat Raghadan, $20^{\circ} 34.25^{\prime} \mathrm{N}, 41^{\circ} 45.11^{\prime} \mathrm{E}, 13 . \mathrm{V} .2011$, SN, Fadl et al., 2exs; Wadi Turubah, $20^{\circ} 14.37^{\prime} \mathrm{N}, 4^{\circ} 15.23^{\prime} \mathrm{E}, 14 . X .2010$, SN, Fadl et al.,

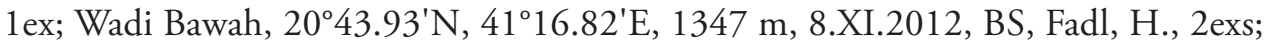
8.XI.2012, HP, Abdel-Dayem, M., 1ex; Wadi Turubah, $20^{\circ} 14.37^{\prime} \mathrm{N}, 41^{\circ} 15.23^{\prime} \mathrm{E}$, 14.V.2011, SN, Fadl et al., 1ex; 3.VI.2012, BS, Al Ansi, A., 1 क 5exs; 3.VI.2012, SN, Al Ansi, A., 10'; Thee Ain, $19^{\circ} 55.78^{\prime} \mathrm{N}, 4^{\circ} 26.60^{\prime} \mathrm{E}, 741 \mathrm{~m}, 13 . X .2010$, SN, Fadl et al., 1ex; 18.V.2010, SN, Al Dhafer et al., 3exs; 18.V.2010, BS, Sharaf, M., 1ex; Wadi Milan, $19^{\circ} 50.79^{\prime} \mathrm{N}, 41^{\circ} 21.01^{\prime} \mathrm{E}, 481 \mathrm{~m}$, 7.III.2013, SN, Al Harbi et al., 14exs; Bani Farwah, $20^{\circ} 02.13^{\prime} \mathrm{N}, 41^{\circ} 29.37^{\prime} \mathrm{E}, 2065$ m, 25.IV.2013, BS, Al Ansi et al., 1ex; Rahwan, $10 \mathrm{~km}$ outside of Baha, 20 $04.03^{\prime} \mathrm{N}, 41^{\circ} 27.01^{\prime} \mathrm{E}, 2272 \mathrm{~m}$, 24.IV.2013, BS,

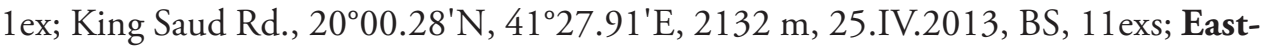

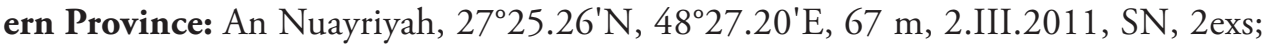
Jizan: Al Aydabi, $17^{\circ} 21.67^{\prime} \mathrm{N}, 43^{\circ} 02.70^{\prime} \mathrm{E}, 272 \mathrm{~m}$, 22.V.2012, SN, Al Ansi, A., 1ex; Al Aydabi, Jaorat Aiban, $17^{\circ} 25.53^{\prime} \mathrm{N}, 43^{\circ} 03.50^{\prime} \mathrm{E}, 343$ m, 23.V.2012, BS, Al Ansi, A., 1ex; Al Muruj Village, 1647.34'N, 4245.14'E, 11.III.2010, SN, Al Dhafer, H. and El Gharabawy, A., 2exs; Al Aridah, Jizan Dam, $17^{\circ} 02.62^{\prime} \mathrm{N}, 42^{\circ} 59.36^{\prime} \mathrm{E}, 187 \mathrm{~m}$,

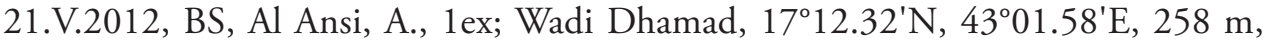
11.XI.2012, LT, Abdel-Dayem, M., 1ex; Najran:Wadi Shuaib Barran, 17²8.94'N, 4405.52'E, 1325 m, 16.I.2013, BS, Al Ansi, A., 10exs; Hubuna, Wadi Hubuna, $17^{\circ} 50.41^{\prime} \mathrm{N}, 44^{\circ} 02.29^{\prime} \mathrm{E}, 1309$ m, 14.I.2013, SN, Al Ansi, A., 2exs; Riyadh:

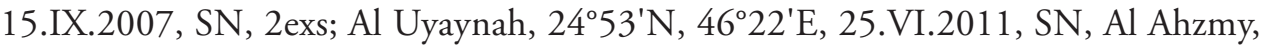
H., 1ex; 30.XI.2010, SN, Al Ansi, A., 2exs; 24.III.2006, SN, Al Homaidi, O., 1 \%; 27.IV.2011, SN, Al Jreed, Y., 1ex; Ad Diriyah, $2^{\circ} 40^{\prime} \mathrm{N}, 46^{\circ} 35^{\prime} \mathrm{E}, 7 . I V .2010, \mathrm{SN}$, Hazazi, T., 1ex; 17.III, SN, 1ex; 5.II.1986, SN, 1ex; 3.X.1991, SN, 1ex; Riyadh, IX.1989, SN, 1ex; 16.X.2003, SN, Al Dhobaib, N., 1ex; Al Kharj, 20²4'N, 46²2'E, 24.III.2010, SN, Al Hashel, A., 1ex; Sajir, 245․ $23^{\prime} \mathrm{N}, 45^{\circ} 42.39^{\prime} \mathrm{E}, 17 . X I .2011$,

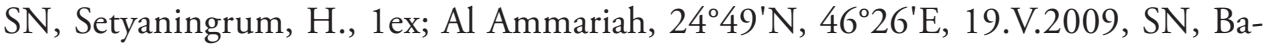
ziad, A., 1ex; 21.X.2009, SN, Abdel-Gayed, A., 1ex; 19.XII.2002, SN, Saad, F., 1ex; Dirab, 2330'N, 4651'E, 2.X.1989, SN, 1ex; 18.IV.1982, SN, 1ex; 20.XI.1986, SN, Amro, 1ex; 28.III.2010, ST, Al Dhafer et al., 1ex; 7.IV.1987, SN, 1ex; 24.XI.1986, SN, 1ex; 21.IV.1987, SN, 1ex; 13.X.2010, ST, Setyaningrum et al., 1ex; 25.IV.2010, ST, Al Dhafer et al., 1ex; 26.IV.2012, SN, Al Qahtani, R., 1ex; 9.V.2012, SN, Al Qahtani, R., 1ex; Al Waseel, 24²48.40'N, 4630.42'E, 25.III.2004, SN, Al Aati, M., 
1ex; 11.V.2010, SN, Khan, M., 1ex; As Sullayyil Al Aflag Rd. 69 Km before Al Aflag, $21^{\circ} 22.92^{\prime} \mathrm{N}, 46^{\circ} 05.29^{\prime} \mathrm{E}, 653 \mathrm{~m}, 17 . \mathrm{I} .2013$, BS, Al Ansi, A., 1ex; Rawdet Khoraim, $25^{\circ} 22.98^{\prime} \mathrm{N}, 47^{\circ} 16.71$ 'E, $559 \mathrm{~m}$, 18.II.2012, SN, 1ex; 30.VI.2012, SN, 1ex; 27.V.2012, LT, 1ex; 26.V.2012, MT, 1ex; 28.VII.2012, MT, 1ex; 27.VIII.2012, MT, 1ex; 27.VIII.2012, PT, 1ex; Tabuk: Tabuk-Dhuba Rd., $28^{\circ} 18.39^{\prime} \mathrm{N}, 36^{\circ} 02.87^{\prime} \mathrm{E}$,

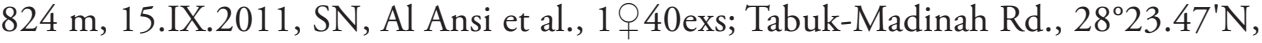
36 51.96'E, 808 m, 14.IX.2011, SN, 10ex; Tabuk-Taima Rd., $27^{\circ} 43.51^{\prime} \mathrm{N}$, $38^{\circ} 12.94^{\prime} \mathrm{E}, 16 . \mathrm{IX} .2011$, SN, 1ex; Tabuk-Dhuba Rd., $28^{\circ} 18.39^{\prime} \mathrm{N}, 36^{\circ} 02.87^{\prime} \mathrm{E}$, 824 m, 15.IX.2011, SN, 3exs.

Local distribution. It is found in Asir, Baha, Eastern, Jizan, Makkah, Najran, Riyadh, and Tabuk provinces and previously reported from Asir by Fürsch (1979) as $S$. levaillanti and from Riyadh by Abdel-Dayem et al. (2017).

World distribution. Asia: AE, AF, IN, IQ, PAL, JA (Bonin, Ryukyus), JO, KU, LE, OM, NP, PA, SA, SD, SY, TAI, TR, and UP; Europe: AZ, GR, IT, PT, and SP; North Africa: CI, EG, and MR; AFR, AUR, and ORR (Kovár 2007).

\section{Scymnus (Scymnus) scapuliferus Mulsant, 1850*}

Figure 7

Scymnus scapuliferus Mulsant, 1850: 968.

Diagnosis. Body is elongated and convex; head is pale in males and black with anterior yellow border in females. Pronotum is black except for a narrow testaceous border on the anterior margin. Elytra are black with yellowish, reddish or pale, nearly quadrangular shoulder spot along the proximal two-thirds. Specimens of this species were found mostly in natural ecosystems, although it has been reported as a predator of the cowpea aphid, Aphis craccivora Koch, 1854, in Nigeria (Waterhouse 1998).

Materials examined. Asir: Al Majardah, Wadi Yabah, $19^{\circ} 16.27^{\prime} \mathrm{N}, 41^{\circ} 48.46^{\prime} \mathrm{E}$,

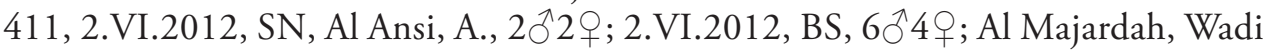
Khat, $19^{\circ} 05.37^{\prime} \mathrm{N}, 41^{\circ} 58.37^{\prime} \mathrm{E}, 13$. III.2012, BS, $10^{\top}$; 31.V.2012, BS, $10^{\top}$; Wadi Gala, $20^{\circ} 08.08^{\prime} \mathrm{N}, 41^{\circ} 20.56^{\prime} \mathrm{E}, 16 . \mathrm{V} .2011$, SN, Fadl et al., 10'; Baha: Thee Ain, $19^{\circ} 55.78^{\prime} \mathrm{N}, 41^{\circ} 26.60^{\prime} \mathrm{E}, 741 \mathrm{~m}, 11 . \mathrm{V} .2011, \mathrm{SN}, 4$ \% ; 15.V.2011, SN, 202웅 10.III.2012, SN, 1 ; 25.IV.2013, BS, Al Ansi et al., 10َ' Al Mikhwah, 19²9.44'N, $41^{\circ} 22.85^{\prime} \mathrm{E}, 430 \mathrm{~m}, 18 . \mathrm{V} .2010$, SN, Al Dhafer et al., 2 ; ; Raghadan, $20^{\circ} 34.25^{\prime} \mathrm{N}$, 41\%45.11'E, 13.V.2011, SN, Fadl et al., 1 $\$$; Jizan: Jizan-Ahd Al Masareha Rd., $17^{\circ} 02.28^{\prime} \mathrm{N}, 42^{\circ} 52.38^{\prime} \mathrm{E}, 11 . \mathrm{III} .2010$, LT, Al Dhafer et al., 10'; 12.III.2010, LT, Al Dhafer et al., 1 \% ; King Faisal Sport City, $16^{\circ} 48.23^{\prime}$ N, $42^{\circ} 59.49^{\prime} E, 11$ m, 20.V.2012,

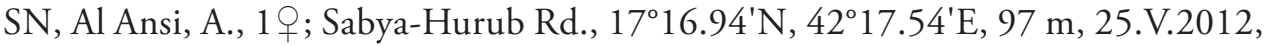
$\mathrm{SN}, \mathrm{Al}$ Ansi, A., 1 ㅇ․

Local distribution. It was collected from Asir, Baha, and Jizan.

World distribution. This species has been reported from South, Central, and West Africa; Yemen (Raimundo and van Harten 2000); new country record for Saudi Arabia. 


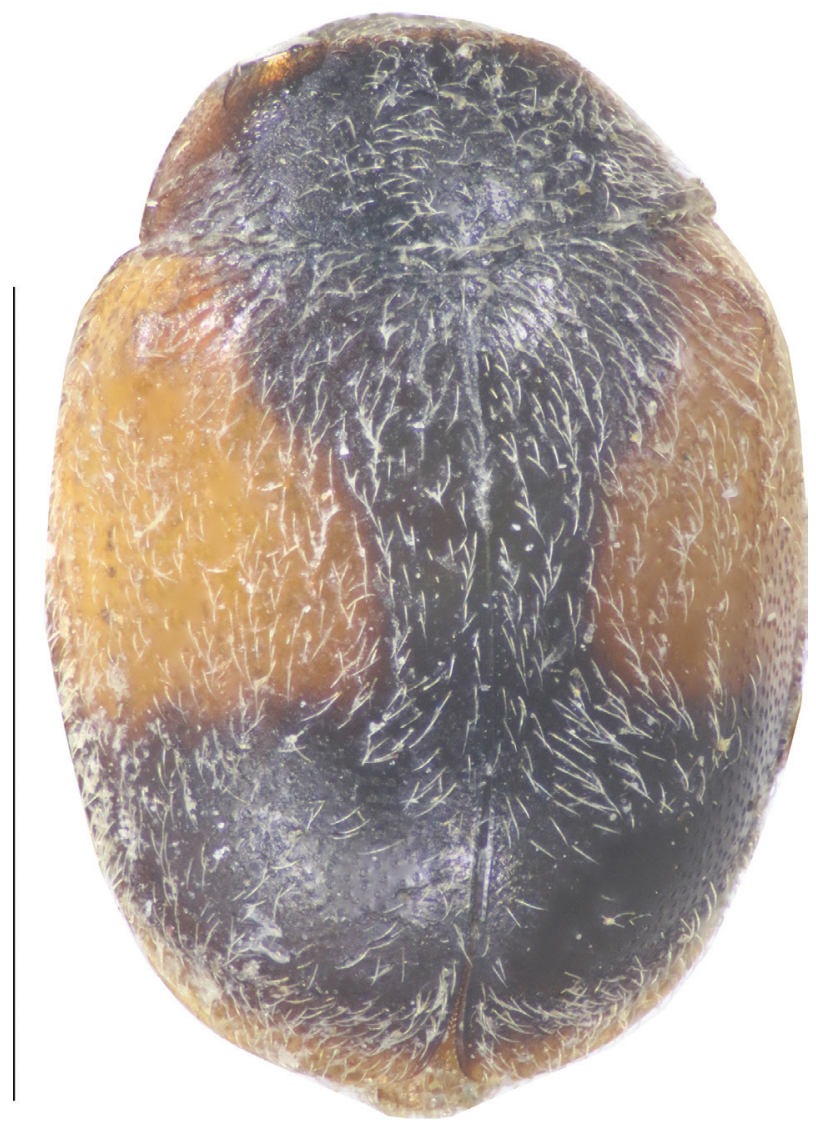

Figure 7. Dorsal view of Scymnus(Scymnus) scapuliferus. Scale bar: $1 \mathrm{~mm}$.

\section{Stethorini Dobzhansky, 1924}

Stethorus Weise, 1885

\section{Stethorus gilvifrons (Mulsant, 1850)}

Scymnus gilvifrons Mulsant, 1850: 995.

Remark. This species is known as a predator of spider mites (Martin 1972; AbuThuraya 1982; Talhouk 1982; Raimundo and van Harten 2000) and is generally found in natural ecosystems.

Materials examined. Baha: Wadi Al Zaraeb, $20^{\circ} 03.60^{\prime} \mathrm{N}, 41^{\circ} 23.19^{\prime} \mathrm{E}, 2123 \mathrm{~m}$, 15.V.2010, BS, Sharaf, M., 1ex; Wadi Al Lehian, $20^{\circ} 09.78^{\prime} \mathrm{N}, 41^{\circ} 36.18^{\prime} \mathrm{E}, 1641 \mathrm{~m}$, 25.IV.2013, BS, Al Ansi, A. and Al Harbi, M., 155exs; Al Lieshtah Village 20 km outside of Baha, $20^{\circ} 12.16^{\prime} \mathrm{N}, 41^{\circ} 22.21^{\prime} \mathrm{E}, 2231 \mathrm{~m}, 26 . \mathrm{IV} .2013$, BS, Al Ansi, A. and

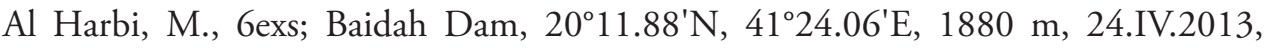
BS, Al Ansi, A. and Al Harbi, M., 1ex; Eastern Province: Al Ahsa, $22^{\circ} 17.53^{\prime} \mathrm{N}$, 
5040.46'E, 28.XII.1982, Al Shuaaei, M., ANMA, 1ex and Najran: Rijla Wadi Najran, $17^{\circ} 31.56^{\prime} \mathrm{N}, 44^{\circ} 13.65^{\prime} \mathrm{E}, 1257$ m, 15.I.2013, BS, Al Ansi, A., $4{ }^{\top} 126$ exs; Wadi Shuaib Barran, $17^{\circ} 28.94^{\prime} \mathrm{N}, 4^{\circ} 05.52^{\prime} \mathrm{E}, 1325$ m, 16.I.2013, BS, Al Ansi, A., 38exs.

Local distribution. It was collected from Baha, Eastern Province, and Najran and was previously reported by Fürsch (1979) from Asir and Riyadh, by Abu-Thuraya (1982) from Eastern Province and Riyadh, and by Talhouk (1982) from Eastern Province, Riyadh, and Makkah and was listed by Martin (1972).

World distribution. Asia: AF, CY, IN, IQ, KA, LE, PA, SA, SY, and TR; Europe: AB, AR, CR, BU, FR, GR, and IT; North Africa: EG; ORR (Kovár 2007) and YE (Raimundo and van Harten 2000); AE (Raimundo et al. 2007).

\section{Stethorus endrodyi Fürsch, 1970*}

Figure 8

Stethorus endrodyi Fürsch, 1970: 98.

Diagnosis. Body is elongated and oval, with dorsal and ventral sides dark, except for the mouth parts, antennae, legs, and frons, which are testaceous; dorsal surface with

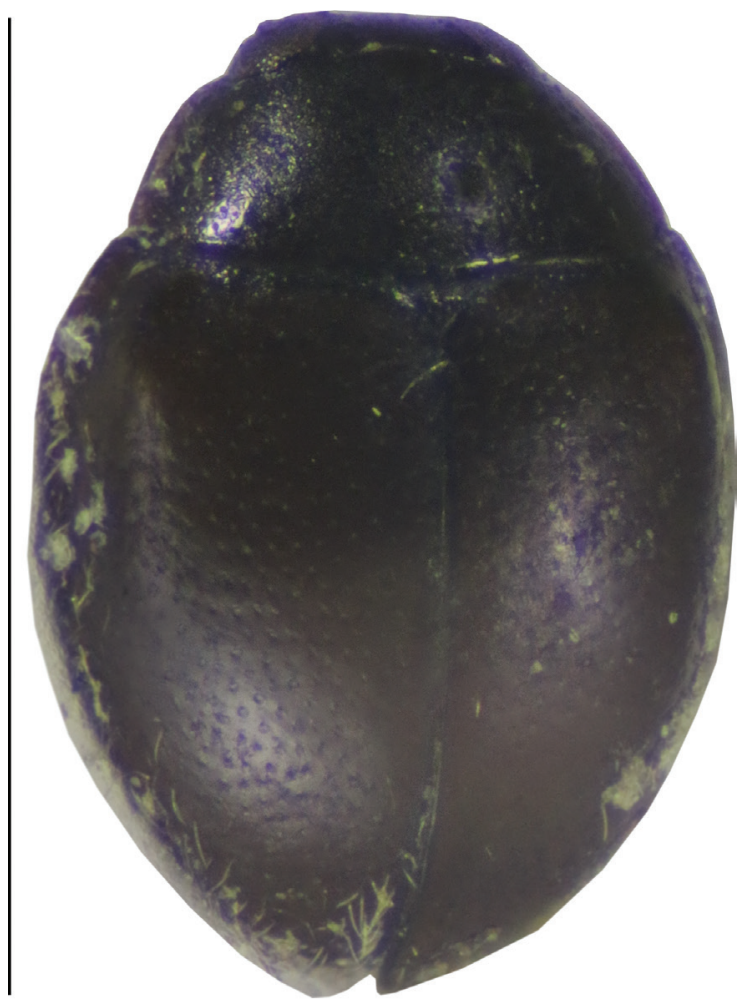

Figure 8. Dorsal view of Stethorus endrodyi. Scale bar: $1 \mathrm{~mm}$. 
short, white, semi-erect, not dense pubescence; pronotum is very sparsely and finely punctate (Fürsch 1970).

Remark. This is a very rare species, and only one specimen was collected from wild vegetation throughout the study period. It is also not common in the rest of the world.

Material examined. Asir: Al Majaredah Wadi Yabah, $19^{\circ} 16.27^{\prime} \mathrm{N}, 41^{\circ} 48.46^{\prime} \mathrm{E}$, 411, 2.VI.2012, SN, Al Ansi, A., 1ex.

Local distribution. Individuals of this species were collected from Asir province.

World distribution. YE, Congo, MO, Ivory Coast, Ghana, Angola, and Malawi (Raimundo et al. 2006); new country record for Saudi Arabia.

\section{Sticholotidini Weise, 1901}

Pharoscymnus Bedel, 1906

\section{Pharoscymnus arabicus Fürsch, 1979}

Pharoscymnus arabicus Fürsch, 1979: 238.

Remark. A rare species that has limited geographical distribution and found only in some countries of the Middle East. It was not collected during the study period.

Local distribution. It was previously reported from Asir by Fürsch (1979).

World distribution. Asia: AE, IN, and SA (Kovár 2007).

\section{Pharoscymnus c-luteum (Sicard, 1907)}

Pharus c-luteum Sicard, 1907: 417.

Remark. This is a rare species, only known in the Arabian Peninsula, and found as a predator of armoured scale insects (Hemiptera: Diaspididae) (Raimundo and van Harten 2000).

Materials examined. Asir: Al Majardah, Wadi Khat, $19^{\circ} 05.37^{\prime} \mathrm{N}, 41^{\circ} 58.37^{\prime} \mathrm{E}$, 31.V.2012, BS, Al Dhafer et al., 2exs; Al Majardah, Wadi Al Talalie, $19^{\circ} 05.19^{\prime} \mathrm{N}$, 41 47.78'E, 286 m, 1.VI.2012, BS, Al Ansi, A., 1ex; Ahd Rifidh, $18^{\circ} 06.33^{\prime} \mathrm{N}$, 42 $53.82^{\prime} \mathrm{E}, 16 . \mathrm{I} .2013$, BS, Al Ansi et al., 2exs; Al Majardah, Wadi Yabah, $19^{\circ} 16.27^{\prime} \mathrm{N}, 41^{\circ} 48.46^{\prime} \mathrm{E}, 411$, 2.IV.2012, BS, Al Ansi, A., $3{ }^{\top} 24 \mathrm{exs}$; 2.IV.2012, LT, Al Ansi, A., 1ex; 2.IV.2012, SN, Al Ansi, A., 1ex; Al Majardah, Wadi Thalooth Al Mandhar, 42 01.12'E, 433 m, 31.V.2012, BS, Al Ansi, A., Gexs; Baha: Wadi Turubah, $20^{\circ} 14.37^{\prime} \mathrm{N}, 4^{\circ} 15.23^{\prime} \mathrm{E}$, 27.IX.2013, Al Dhafer, H., 2exs; Thee Ain, $19^{\circ} 55.78^{\prime} \mathrm{N}, 4^{\circ} 26.60^{\prime} \mathrm{E}, 741 \mathrm{~m}, 10 . I I I .2012$, BS, Al Dhafer et al., 1ex; 25.IV.2013, BS, Al Ansi et al., 3exs; 3.VI.2012, BS, Al Ansi, A., 3exs; Al Quhman Village, 5 km before Al Atawilah, $20^{\circ} 19.23^{\prime} \mathrm{N}, 41^{\circ} 19.48^{\prime} \mathrm{E}, 2014 \mathrm{~m}$, 24.IV.2013, BS, Al Ansi et al.,

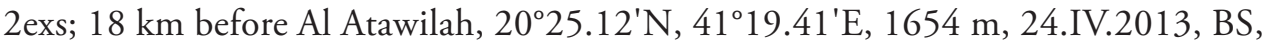

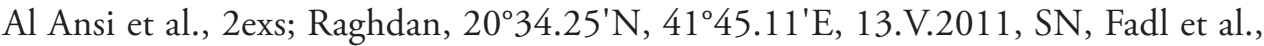
1ex; Sad Medhas, $20^{\circ} 13.26^{\prime} \mathrm{N}, 41^{\circ} 16.53^{\prime} \mathrm{E}, 1781 \mathrm{~m}$, 9.III.2012, SN, Al Dhfer et al., 
4exs; Jizan: Hurub Wadi Hurub, $17^{\circ} 43.12^{\prime} \mathrm{N}, 42^{\circ} 57.88^{\prime} \mathrm{E}, 398$ m, 24.V.2012, BS, Al Ansi, A., 5exs; Hurub Wadi Qasi, $17^{\circ} 26.52^{\prime} \mathrm{N}, 42^{\circ} 57.32^{\prime} \mathrm{E}, 284 \mathrm{~m}, 24 . V .2012$, BS, Al Ansi, A., 1ex.

Local distribution. Specimens of this species were collected from Asir, Baha, and Jizan. It was previously reported by Fürsch (1979) from Asir province.

World distribution. Asia: SA and YE (Kovár 2007).

\section{Pharoscymnus fleischeri (J. Weise, 1883)*}

Figure 9

Pharus fleischeri J. Weise, 1883: 67.

Remark. This is a rare species with limited geographical range and found only in the Middle East and Germany. It was attracted to light in the month of May.

Material examined. Riyadh: Al Hariq-Ar Raayn Rd., $23^{\circ} 33.88^{\prime} \mathrm{N}, 46^{\circ} 22.53^{\prime} \mathrm{E}$, 737 m, 8.V.2012, LT, Al Ansi, A., 1ex.

Local distribution. Riyadh province.

World distribution. Asia: IN and TR and newly recorded from SA. Europe: GR (Kováŕ 2007).

\section{Pharoscymnus numidicus (Pic, 1900)}

Pharus numidicus Pic, 1900: 91.

Remark. This species was found in the natural ecosystem of the KSA. It is a very good biological control agent and has been introduced into Morocco for the control of Parlatoria blanchardi (Targioni Tozzetti, 1892) (Raimundo and van Harten 2000). Specimens were collected using beating sheet.

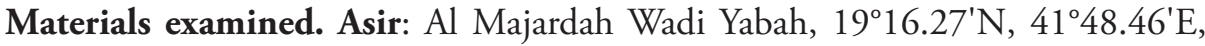
411, 2.VI.2012, BS, Al Ansi, A., 1+13exs; 2.VI.2012, SN, Al Ansi, A., 1ex; 12.III.2012, SN, Al Ansi, A., 2exs; Al Majardah Wadi Thalooth Al Mandhar, 4201.12'E, 433 m, 31.V.2012, BS, Al Ansi, A., 5exs; Al Majardah, Wadi Qanunah, $19^{\circ} 24.67^{\prime} \mathrm{N}, 41^{\circ} 36.39^{\prime} \mathrm{E}, 348 \mathrm{~m}, 11 . I I I .2012$, SN, Al Dhafer et al., $1{ }^{\top} 36$ exs; Al Majardah, Wadi Baqrah, $18^{\circ} 47.57^{\prime} \mathrm{N}, 42^{\circ} 01.12^{\prime} \mathrm{E}, 433 \mathrm{~m}, 10 . X I .2012$, BS, Fadl, H., 2exs; Al Majardah, Wadi Qanunah, 19²4.67'N, 4136.39'E, 348 m, 11.III.2012, LT, Al Dhafer et al., 1ex; Baha: Thee Ain, $19^{\circ} 55.78^{\prime} \mathrm{N}, 4^{\circ} 26.60^{\prime} \mathrm{E}, 741 \mathrm{~m}, 18 . V .2010$, BS, Sharaf, M., 1ex; 3.VI.2012, BS, Al Ansi, A., 1ex; Jizan: Al Aydabi Wadi Qasi, $17^{\circ} 26.52^{\prime} \mathrm{N}, 42^{\circ} 57.32^{\prime} \mathrm{E}, 284 \mathrm{~m}, 23 . V .2012$, BS, Al Ansi, A., 195exs; Hurub Wadi Qasi, $17^{\circ} 26.52^{\prime} \mathrm{N}, 42^{\circ} 57.32^{\prime} \mathrm{E}, 284 \mathrm{~m}, 24 . V .2012$, BS, Al Ansi, A., $10^{\Uparrow} 4 \mathrm{exs}$; Aiban Sabya Rd. Wadi Shahdan, $17^{\circ} 28.27^{\prime} \mathrm{N}, 42^{\circ} 51.19^{\prime} \mathrm{E}$, 433 m, 13.XI.2012, BS, Fadl, H.,

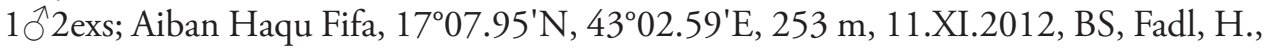




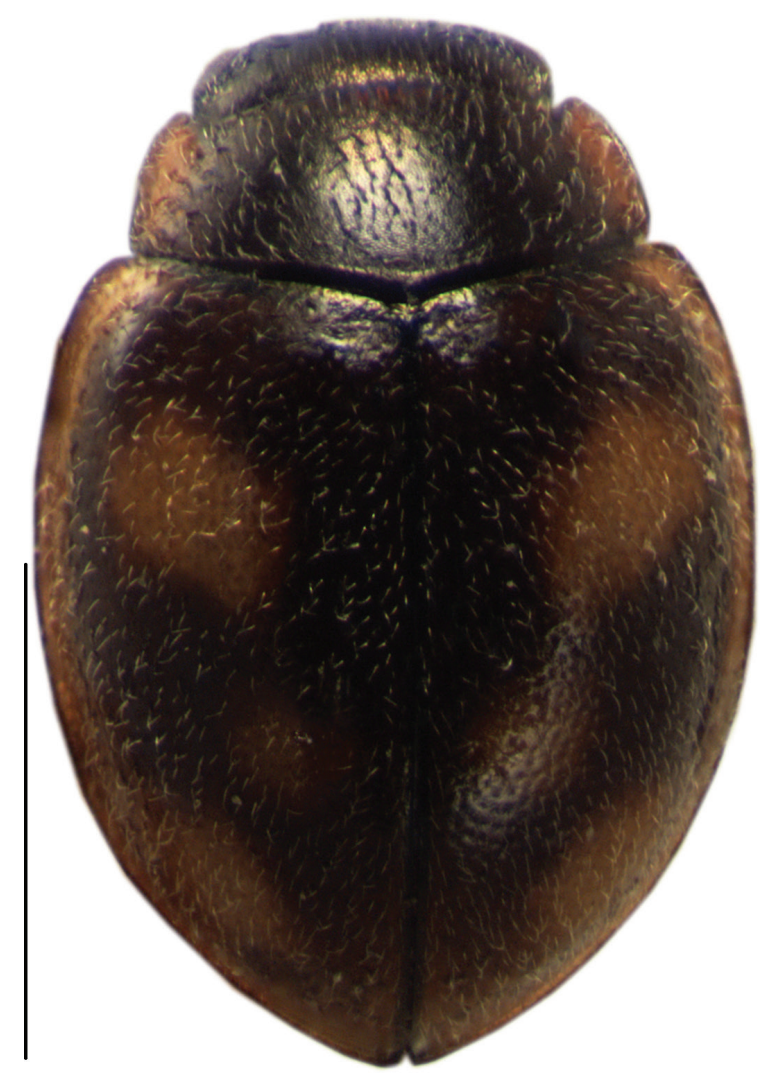

Figure 9. Dorsal view of Pharoscymnus fleischeri. Scale bar: $1 \mathrm{~mm}$.

1ex; Al Aaridah Wadi Al Rad, $17^{\circ} 04.10^{\prime} \mathrm{N}, 43^{\circ} 04.33^{\prime} \mathrm{E}, 192 \mathrm{~m}, 21 . \mathrm{V} .2012$, BS, Al Ansi, A., 5exs; Hurub Wadi Hurub, $17^{\circ} 43.12^{\prime} \mathrm{N}, 42^{\circ} 57.88^{\prime} \mathrm{E}, 398$ m, 24.V.2012, BS, Al Ansi, A., 1ex; Al Aaridah Jizan Dam, $17^{\circ} 02.62^{\prime} \mathrm{N}, 42^{\circ} 59.36^{\prime} \mathrm{E}, 187 \mathrm{~m}, 21 . \mathrm{V} .2012$, BS, Al Ansi, A., 1ex; Wadi Shuaib Barran, $17^{\circ} 28.94^{\prime} \mathrm{N}, 4^{\circ} 05.52^{\prime} \mathrm{E}, 1325$ m, 16.I.2013, BS, Al Ansi et al., 1ex.

Local distribution. Members of this species were collected from Asir, Baha, and Jizan, and it was previously reported by Fürsch (1979) from Asir and Riyadh.

World distribution. Asia: PAL, JO, and SA; North Africa: AG, EG, LB, and TU; AFR (Kovár 2007); AE (Raimundo et al. 2007).

\section{Pharoscymnus ovoideus Sicard, 1929}

Pharoscymnus ovoideus Sicard, 1929: 61.

Remark. This species is a predator of scale insects (Martin 1972; Montaigne and Maouloud 1981; Abu-Thuraya 1982). 
Materials examined. Riyadh: Riyadh, 20.VI.1981, Talhouk et al., ANMA, 3exs, det. Fürsch 1967, 1968; Al Hariq Ar Rayn Rd., 2333.88'N, 46²2.53'E, 737 m, 8.V.2012, LT, Al Ansi, A., 1ex.

Local distribution. It has been recorded in Riyadh and was previously reported by Abu-Thuraya (1982) and listed by Martin (1972).

World distribution. Asia: IN, JO, and SY; North Africa: AG, MO, and TU; AFR (Kovář 2007), SA (Abu-Thuraya 1982); AE (Raimundo et al. 2007).

\section{Pharoscymnus pharoides (Marsuel, 1868)}

Scymnus pharoides Marsuel, 1868: 215.

Remark. Presence of this species was not confirmed, except in the catalogue of Kovár (2007) with insufficient details about its distribution in the KSA. Moreover, not a single specimen was collected during or after the study period.

World distribution. Asia: IN, PAL, SA, SY, and TR; North Africa: EG and LB; AFR (Kovář 2007).

\section{Pharoscymnus setulosus (Chevrolat, 1861)}

Pharus setulosus Chevrolat, 1861: 269.

Remark. No fresh material could be collected to examine this species. Two specimens from the collection of ANMA, collected by R.D. Pope in 1966, were examined. This species was found on a red scale insect, Aonidiella sp., according to the information described by R.D. Pope (1966), Martin (1972) and AbuThuraya (1982).

Material examined. Eastern Province: Hafuf, $25^{\circ} 15^{\prime} \mathrm{N}, 49^{\circ} 32^{\prime} \mathrm{E}, \mathrm{VI} .1966$, ANMA, 2exs, det. Pope, 1966.

Local distribution. Specimens were collected from the Eastern province of SA by Pope in 1966 and was also reported by Fürsch (1979) from wadi Awsat and AbuThuraya (1982) from Eastern Province and was listed by Martin (1972).

World distribution. Asia: AE, IN, PAL, JO, and SA; Europe: SP; North Africa: AG, EG, LB, MO, and TU (Kovár 2007).

\section{Pharoscymnus smirnovi Dobzhanskiy, 1927}

Pharoscymnus smirnovi Dobzhanskiy, 1927: 240.

Remark. This is a rare species with limited geographical distribution.

Material examined. Riyadh: Riyadh, 18.IV.1978, Talhouk et al., ANMA, 1 ex. 
Local distribution. Riyadh province.

World distribution. Asia: SA; Europe: AB, GG (Kovář 2007).

\section{Pharoscymnus tristiculus Sicard, 1907}

Pharus tristiculus Sicard, 1907: 418.

Remark. No fresh material could be collected to examine this species. Two specimens from the collection of ANMA, collected by Pope in 1970, were examined. This species was found on citrus scale insects (Hemiptera) according to the information provided by Pope (1970).

Material examined. Al Madinah: Ovaida farm, $24^{\circ} 25.58^{\prime} \mathrm{N}, 39^{\circ} 36.29^{\prime} \mathrm{E}, 15$. IX.1969, SN, ANMA, 2exs, on citrus scale insects, det. Pope 1970.

Local distribution. Specimens were collected from Al Madinah and were also reported by Abu-Thuraya (1982) from Al Madinah.

World distribution. Asia: YE; AFR (Kovár 2007); SA (Abu-Thuraya 1982).

\section{Microweiseinae Leng, 1920}

Serangiini Pope, 1962

Serangium Blackburn, 1889

\section{Serangium buettikeri Fürsch, 2000}

Serangium buettikeri Fürsch, 2000 (18): 233.

Remark. This species has been recorded only in the Arabian Peninsula and is a predator of the citrus blackfly Aleurocanthus woglumi Ashby, 1915 (Raimundo and van Harten 2000). Individuals were collected from Ricinus communis L. using sweep nets.

Materials examined. Asir: Wadi Khat, $19^{\circ} 05.37^{\prime} \mathrm{N}, 41^{\circ} 58.37^{\prime} \mathrm{E}, 13 . \mathrm{III} .2012$, BS, Al Dhafer et al., 2exs; Baha: Thee Ain, $19^{\circ} 55.78^{\prime} \mathrm{N}, 41^{\circ} 26.60^{\prime} \mathrm{E}, 741 \mathrm{~m}, 10 . \mathrm{III} .2012$, BS, Al Dhafer et al., 1ex; 11.IV.2016, BS, Al Ansi et al., 1ex; Najran: Rijla, Wadi Najran $17^{\circ} 31.56^{\prime} \mathrm{N}, 4^{\circ} 13.65^{\prime} \mathrm{E}, 1257$ m, 15.I.2013, BS, Al Ansi, A., 4ํ2우 $90 \mathrm{exs,}$ on $R$. communis L.; 15.I.2013, SN, Al Ansi, A., 1ex, on R. communis L.; Wadi Shuaib Barran $17^{\circ} 28.94^{\prime} \mathrm{N}, 4^{\circ} 05.52^{\prime} \mathrm{E}, 1325$ m, 16.I.2013, BS, Al Ansi, A., 1ex.

Local distribution. Specimens of this species were reported from Asir, Baha, and Najran provinces and previously reported by Raimundo and van Harten (2000) from Asir.

World distribution. Asia: SA and YE (Kovár 2007).

\section{Discussion}

Data on the coccinellid fauna of the KSA are scarce. Fürsch (1979), who provided a key for 35 species, did not provide any precise locality information for eight of the 
species. Subequently, Kovár (2007) listed 41 species from the KSA but without localities. Before the present study, 56 species were reported in the KSA (Table 2), and the present survey has added eight new records, including $C$. lunata lunata, $C$. arcuatus, $N$. ornatulus, $N$. nigricans, P. fleischeri, $R$. yemenensis, S. scapuliferus, and S. endrodyi, for the country, one new genus record, Clitostethus, and presented a list of 65 species. Despite extensive exploration of the country, 14 previously known species were not collected during the present study, which included six species with known localitydata, $N$. conjunctus (from the village Qaaraah near Khamis Mushayt, Wadi Johan Abha, Wadi Maraba, 8-20 km from the Abha-Taif Rd.); N. fenestratus (from Wadi Shija; Wadi Hanifah; Wadi Khumra; 28 km Abha-Jizan Rd.; Wadi Ad Dilla; Wadi Mizbil and Salbukh); S. latemaculatus (from Khureis); S. arabicus (collected Thanomah; 8-20 km from the Abha-Taif Rd.; village Qaaraah near Khamis Mushayt); P. arabicus (from Wadi Johan Abha); and T. arabicus (from the village Qaaraah near Khamis Mushayt and El Hejdaz), and eight species from unknown localities.

\section{Acknowledgments}

The authors would like to express their deepest gratitude to the team of King Saud University Museum of Arthropods for their assistance in field work, technical support, and processing of specimens in the laboratory. Special thanks to the Arabian National Museum of Arthropods, Ministry of Environment, Water and Agriculture, Riyadh, Saudi Arabia, for providing us the reference collection and preserved collection to examine. Thanks to Prof. Boris Kondratieff, Department of Bioagricultural Sciences and Pest Management, Colorado State University, Fort Collins, Colorado, USA, for his critical review and valuable comments. This research was funded by the Deanship of Scientific Research at Princess Nourah bint Abdulrahman University through the Fast-track Research Funding Program. The authors thank the Deanship of Scientific Research and RSSU at King Saud University for their technical support.

\section{References}

Abu-Thuraya NH (1982) General Survey: Agricultural Pests in Saudi Arabia. Ministry of Agriculture and Water, Riyadh, Kingdom of Saudi Arabia, 266 pp.

Al Akkad MK (1979) Studies on family Coccinellidae as known to exist in Egypt. Unpublished M.Sc. thesis, Al Azhar University, Cairo, 135 pp.

Alizadeh MS, Mossadegh MS, Esfandiari M (2013) Natural enemies of Maconellicoccus hirsutus

(Green) (Hemiptera: Pseudococcidae) and their population fluctuations in Ahvaz, southwest of Iran. Journal of Crop Protection 2(1): 13-21.

Alfieri A (1976) The Coleoptera of Egypt. Mémoires de la société entomologique d'Egypte 5: 133-287.

Awamelah RA, Al Antary TM, Bilal HM (2009) Survey of natural enemies of fig wax scale Ceroplastes rusci L. (Homoptera: Coccidae) and seasonal abundance of the parasitoid 
Scutellista caerulea Fonscolombe (Hymenoptera: Pteromalidae) in Jordan. Jordan Journal of Agriculture Sciences 5(4): 434-445.

Abdel-Dayem MS, Fadl HH, El-Torkey AM, Elgharbawy AA, Aldryhim YN, Kondratieff BC, Al Ansi AN, Aldhafer HM (2017) The beetle fauna (Insecta, Coleoptera) of the Rawdhat Khorim National Park, Central Saudi Arabia. ZooKeys 653: 1-78. https://doi. org/10.3897/zookeys.653.10252

Beccari F (1971) Contribution to knowledge of the entomofauna of Saudi Arabia. First list of insects, mites and nematodes. Rivista di Agricoltura Subtropicale e Tropicale 65(4/6): $178-211$.

Biranvand A, Nedved O, Tomaszewska W, Al Ansi A, Fekrat L, Haghghadam ZM, Khormizi MZ, Noorinahad S, Senal D, Shakarami J, Haelewaters D (2019) The genus Harmonia (Coleoptera, Coccinellidae) in the Middle East region. Acta Entomologica Musei Nationalis Pragae 59(1): 163-170. https://doi.org/10.2478/aemnp-2019-0014

Biranvand A, Tomaszewska W, Nedvěd O, Zare Khormizi M, Nicolas V, Canepari C, Shakarami J, Fekrat L, Fürsch H (2017) Review of the tribe Hyperaspidini Mulsant (Coleoptera: Coccinellidae) from Iran. Zootaxa 4236(2): 311-326. https://doi.org/10.11646/ zootaxa.4236.2.6

Davis SR (2009) Morphology of Baridinae and related groups (Coleoptera, Curculionidae). ZooKeys 10: 1-136. https://doi.org/10.3897/zookeys.10.47

El-Hawagry MS, Khalil MW, Sharaf MR, Fadl HH, Aldawood AS (2013) A preliminary study on the insect fauna of Al-Baha Province, Saudi Arabia, with descriptions of two new species. ZooKeys 274: 1-88. https://doi.org/10.3897/zookeys.274.4529

Fürsch H (1970) Über Coccinellidenausbeuten aus dem Vordern Orient. Mittelungen der Münchener Entomologischen Gesellschaft 59(1969): 108-114.

Fürsch H (1979) Insect of Saudi Arabia Coleoptera: Fam. Coccinellidae. Fauna of Saudi Arabia 1: 235-248.

Fürsch H (1989) The Arabian species of the Scymnus (Pulls) guimeti-group (Coleoptera, Coccinellidae). Fauna of Saudi Arabia 10: 113-122.

Fürsch H (1991) Die Epilachnini Afrikas sudlich der Sahara (Coleoptera: Coccinellidae). Entomofauna Zeitschrift fur Entomologie Band 12, Heft 18/1-3: 217-320.

Gillett MPT (2009) Records of the phytophagous ladybird Henosepilachna elaterii orientalis (Zimmerman 1936) from the Al Ain/ Buraimi area of the UAE and Oman (Coleoptera: Coccinellidae). Tribulus 18: 60-61.

Giorgi JA, Vandenberg NJ, McHugh JV, Forrester JA, Ślipiński SA, Miller KB, Shapiro LR, Whiting MF (2009) The evolution of food preferences in Coccinellidae. Biological Control 51: 215-231. https://doi.org/10.1016/j.biocontrol.2009.05.019

Halperin J, Merkl O, Kehat M (1995) An annotated list of the Coccinellidae (Coleoptera) of Israel and adjacent areas. Phytoparasitica 23(2): 127-137. https://doi.org/10.1007/ BF02980972

Hesler LS (2009) An annotated checklist of the lady beetles (Coleoptera: Coccinellidae) of Iowa, U.S.A. Insecta Mundi 91: 1-10.

Hodek I, Honek A (2009) Scale insects, mealybugs, whiteflies and psyllids (Hemiptera, Sternorrhyncha) as prey of ladybirds. Biological Control 51(2): 232-243. https://doi. org/10.1016/j.biocontrol.2009.05.018 
Kapur AP (1948) The genus Tetrabrachys (Lithophilus) with notes on its biology and a key to the species (Coleoptera, Coccinellidae). Transaction of the Royal Entomological Society of London 99: 319-340. https://doi.org/10.1111/j.1365-2311.1948.tb01240.x

Kapur AP (1959) Expedition to South-West Arabia 1937-1938, No.18. Coleoptera: Coccinellidae. British Museum Natural History, London, 275-297.

Khalil N, Mourad A (2005) A contribution to study on tribe Coccinellini (Coccinellidae, Coleoptera) in south of Syria. Damascus University Journal for Basic Sciences 21(4): 153169. [in Arabic]

Khalil N, Mourad A (2006) A preliminary survey of ladybirds (Coccinellidae) on Coniferales in south of Syria. Journal of Basil Al-Assad of Engineering Science 22: 147-165. [in Arabic]

Koch RL (2003) The multicolored Asian lady beetle, Harmonia axyridis: A review of its biology, uses in biological control, and non-target impacts. Journal of Insect Science 3(32): 1-16. https://doi.org/10.1093/jis/3.1.32

Kovář I (2007) Coccinellidae. In Löbl I, Smetana A (Eds) Catalogue of Palaearctic Coleoptera (Vol. 4). Apollo Books, Stenstrup, Denmark, 568-631.

Leather SR, Cooke RCA, Fellowes MDE, Rombe R (1999) Distribution and abundance of ladybirds (Coleoptera: Coccinellidae) in non-crop habitats. European Journal of Entomology 96(1): 23-27.

Magro A, Lecompte E, Magne F, Hemptinne JL, Crouau-Roy B (2010) Phylogeny of ladybirds (Coleoptera: Coccinellidae): Are the subfamilies monophyletic. Molecular Phylogenetics and Evolution 54: 833-848. https://doi.org/10.1016/j.ympev.2009.10.022

Martin HE (1972) Report to the government of Saudi Arabia on research in plant protection FAO. Entomologist FAO/SAO/IF/63 (AGP.TA) 207, 38.

Montaigne MDE, Maouloud FA (1981) Biological control of date palm scale in Mauritania. (Biological control of pests: Its potential in West Africa). Proceedings of an international conference held in Dakar, Senegal, February 9-13, 1981. Dakar, Senegal: USAID, Regional Food Crop Protection Project, 191-204.

Nedvěd O (2020) Ladybird beetles (Coccinellidae) of Central Europe ( $2^{\text {nd }}$ edn.). Academia, Praha, $304 \mathrm{pp}$.

Obrycki JJ, Kring TJ (1998) Predaceous Coccinellidae in biological control. Annual Review of Entomology 43: 295-321. https://doi.org/10.1146/annurev.ento.43.1.295

Pang H, Tang X, Booth RG, Vandenberg N, Forrester J, Mchugh J, Ślipiński A(2020). Revision of The Australian Coccinellidae (Coleoptera). Genus Novius Mulsant of Tribe Noviini. Annales Zoologici 70(1): 1-24. https://doi.org/10.3161/00034541ANZ2020.70.1.001

Raimundo AC, van Harten A (2000) An annotated checklist of the Coccinellidae (Insect: Coleoptera) of Yemen. Fauna of Saudi Arabia 18: 211-243.

Raimundo AC, Fürsch H, van Harten A (2006) Notes on the ladybird beetles (Coleoptera: Coccinellidae) of Yemen, with the description of two new species. Fauna of Arabia 21: 217-245.

Raimundo AC, Fürsch H, van Harten A. (2007) Order Coleoptera, Family Coccinellidae. Arthropod Fauna of the UAE 1: 217-239.

Robertson JA, Ślipiński A, Moulton M, Shockley FW, Giorgi A, Lord NP, Mckenna DD, Tomaszewska W, Forrester J, Miller KB, Whiting MF, Mchugh JV (2015) Phylogeny 
and classification of Cucujoidea and the recognition of a new superfamily Coccinelloidea (Coleoptera: Cucujiformia). Systematic Entomology 40(4): 745-778. https://doi. org/10.1111/syen.12138

Roy H, Migeon A (2010) Ladybeetles (Coccinellidae), Chapter 8.4. BioRisk 4(1): 293-313. https://doi.org/10.3897/biorisk.4.49

Roy HE, Brown PMJ, Adriaens T, Berkvens N, Borges I, Clusella-Trullas S, Comont RF, De Clercq P, Eschen R, Estoup A, Evans EW, Facon B, Gardiner MM, Gil A, Grez AA, Guillemaud T, Haelewaters D, Herz A, Honek A, Howe AG, Hui C, Hutchison WD, Kenis M, Koch RL, Kulfan J, Handley LL, Lombaert E, Loomans A, Losey J, Lukashuk AO, Maes D, Magro A, Murray KM, San Martin G, Martinkova Z, Minnaar IA, Nedved O, OrlovaBienkowskaja MJ, Osawa N, Rabitsch W, Ravn HP, Rondoni G, Rorke SL, Ryndevich SK, Saethre M-G, Sloggett JJ, Soares AO, Stals R, Tinsley MC, Vandereycken A, van Wielink P, Viglášová S, Zach P, Zakharov IA, Zaviezo T, Zhao Z (2016) The harlequin ladybird, Harmonia axyridis: global perspectives on invasion history and ecology. Biological Invasions 18(4): 997-1044. https://doi.org/10.1007/s10530-016-1077-6

Sattar M, Hamed M, Nadeem S (2008) Biology of Coccinella septempunctata L. (Coleoptera: Coccinellidae) and its Predatory Potential on Cotton Aphids, Aphis gossypii Glover (Hemiptera: Aphididae). Pakistan Journal Zoology 40(4): 239-242.

Shalaby F (1962) Contribution to the insect fauna of Saudi Arabia. Bulletin de la Société Entomologique d'Egypte 46: 339-342.

Talhouk AS (1982) Applied zoology in Saudi Arabia a note on the entomophagous fauna of Saudi Arabia. Fauna of Saudi Arabia 4: 529-531.

Tavadjoh Z, Hamzehzarghani H, Alemansoor H, Khalghani J, Vikram A (2009) Biology and feeding behaviour of ladybird, Clitostethus arcuatus, the predator of the ash whitefly, Siphoninus phillyreae, in Fars province, Iran. Journal of Insect Science 10(120): 1-12. https:// doi.org/10.1673/031.010.12001

Vandenberg NJ (2002) Coccinellidae Latreille, 1807. In: Arnett RH, Thomas MC (Eds) American Beetles (Vol. 2). Polyphaga: Scarabaeoidea through Curculionoidea. CRC Press, Boca Raton, 371-389.

Walker DH, Pittaway AR (1987) Insect of Eastern Arabia. Macmillan Nature, 175 pp.

Waterhouse DF (1998) Biological control of insect pests: Southeast Asian prospects, Australian Center for International Agricultural Research. Aciar Monograph No. 51, Canberra, 548 pp. [+ viii, 1 fig., 16 maps.] 